Proceedings of the Institution of Mechanical Engineers, Part H: Journal of Engineering in Medicine

\title{
https://journals.sagepub.com/home/pih
}

Accepted May 31st 2021

\section{UNSTEADY HYBRID NANOPARTICLE-MEDIATED MAGNETO-HEMODYNAMICS AND HEAT TRANSFER THROUGH AN OVERLAPPED STENOTIC ARTERY: BIOMEDICAL DRUG DELIVERY SIMULATION}

\author{
Jayati Tripathi ${ }^{1}$ B. Vasu ${ }^{1}$, O. Anwar Bég ${ }^{2}$ and Rama Subba Reddy Gorla ${ }^{3}$ \\ ${ }^{1}$ Department of Mathematics, Motilal Nehru National Institute of Technology Allahabad, \\ Prayagraj, Uttar Pradesh- 211004, India \\ ${ }^{2}$ Professor of Engineering Science, Department of Mechanical Engineering, School of Science, Engineering \\ and Environment (SEE), Newton building, Salford University, Manchester, M54WT, UK. \\ ${ }^{3}$ Department of Aeronautics and Astronautics, Air Force Institute of Technology, \\ Wright Patterson Air Force Base, Dayton, Ohio 45433, USA \\ *Corresponding author-email: bvasu@mnnit.ac.in
}

\begin{abstract}
Two-dimensional laminar hemodynamics through a diseased artery featuring an overlapped stenosis was simulated theoretically and computationally. This study presented a mathematical model for the unsteady blood flow with hybrid biocompatible nanoparticles (Silver and Gold) inspired by drug delivery applications. A modified Tiwari-Das volume fraction model was adopted for nanoscale effects. Motivated by the magnetohemodynamics effects, a uniform magnetic field was applied in the radial direction to the blood flow. For realistic blood behavior, Reynolds' viscosity model was applied in the formulation to represent the temperature dependency of blood. Fourier's heat conduction law was assumed, and heat generation effects were included. Therefore, the governing equations were an extension of the Navier-Stokes equations with magneto-hydrodynamic body force included. The two-dimensional governing equations were transformed and normalized with appropriate variables, and the mild stenotic approximation was implemented. The strongly nonlinear nature of the resulting dimensionless boundary value problem required a robust numerical method, and therefore the FTCS algorithm was deployed. Validation of solutions for the particular case of constant viscosity and non-magnetic blood flow was included. Using clinically realistic hemodynamic data, comprehensive solutions were presented for silver, and silver-gold hybrid mediated blood flow. A comparison between silver and hybrid nanofluid was also included, emphasizing the use of hybrid nanoparticles for minimizing the hemodynamics. Enhancement in magnetic parameter decelerated the axial blood flow in stenotic region. Colored streamline plots for blood, silver nano-doped blood, and hybrid nano-doped blood were also presented. The simulations were relevant to the diffusion of nano-drugs in magnetic targeted treatment of stenosed arterial diseases.
\end{abstract}

KEYWORDS: Arterial stenosis; Unsteady viscous hemodynamics; Hybrid nanoparticles (Ag-Au/blood); Nanodrug delivery; Finite Difference Method (FDM); Reynolds's viscosity model; Heat transfer; Thermal buoyancy; Wall Shear Stress (WSS). 


\section{Introduction}

Considering the present-day statistics of the World Health Organization, 2017 [1], cardiovascular diseases are responsible, for the majority of cases, of morbidity and mortality across the world. Almost $31 \%$ of total deaths, i.e. 17.9 million, are documented due to be caused by these diseases. Cardiovascular diseases (CVDs) encompass cerebrovascular, rheumatic heart, coronary heart, peripheral and congenital heart disease, among other ailments. The fundamental reason for these diseases is the deposition of cholesterol and low-density lipoproteins inside the lumen, resulting in the hardening of arteries, which is called atherosclerosis. This plaque further grows and narrows the arterial area, manifesting in a hemodynamic constriction, termed a stenosis [2], which reduces the blood supply to different organs of the body. Human blood is a heterogeneous multiphase suspension of blood cells (erythrocytes, leukocytes, platelets,) in plasma. It is universally agreed that plasma is a Newtonian fluid [3]; however, whole blood exhibits non-Newtonian fluid characteristics owing to the suspensions present which significantly alter rheological properties [4]. For blood flow transporting through large arteries, Newtonian behaviour can be assumed. However, in small arteries or capillaries, blood exhibits a strongly non-Newtonian nature. Another critical feature of arterial blood flow is its pulsatile behavior, which was highlighted by Ling and Atebek [5].

A large number of studies have been performed to elaborate the hemodynamics in arteries considering blood as either a Newtonian or non-Newtonian fluid. Chakravarty and Mandal [6] computed the time-dependent non-Newtonian blood flow in an artery with overlapped stenosis with a finite difference technique using an elastic (moving wall) cylindrical tube model and viscoelastic constitutive equation fluid representing blood. Chakravarty and Mandal [7] presented a mathematical model for Newtonian blood flow in a tapered blood vessel using a clinically realistic time-variant shape of the overlapping stenosis present in the arterial lumen and including vascular wall elastic deformability. Mekheimer and El kot [8] considered heat, mass and momentum transfer in Sisko shear-thinning blood flow in stenotic arteries with cross diffusion effects. Riahi et al. [9] computed the variable viscosity blood flow in an overlapping stenotic region. They showed that impedance is elevated with stenosis parameter and shear stress at the throats and at the critical height increase with the severity of the stenosis. Haghighi and Chalak [10] simulated blood flow in constricted arteries using a finite difference method and a Sisko nonNewtonian model and under body acceleration effects. Srivastav [11] analyzed hemodynamics in 
a catheterized stenosed artery with a Newtonian model with permeability effects. Zaman et al. [12] computed the transient magnetized blood flow in a porous saturated overlapping artery. Ali et al. [13] used a finite difference technique to simulate time-dependent shear-thinning blood flow in a tapered artery with a single stenosis. All these studies confirmed the importance of either inclusion of time and/or rheological effects in evaluating more precisely the hemodynamics in constricted blood vessels and the contribution to proliferation of hematological disorders.

The statistics emphasizes the urgent requirements for new and more effective therapies and technologies which can prevent these diseases. In recent years, nanofluids have emerged as a significant development in biomedical engineering. Choi [14] proposed the term "nanofluid," which is defined as a suspension of dissimilar nanoparticles in a base fluid aimed at enhancing thermal (and other) characteristics. The nanoparticles are usually oxides, carbides or metals having high thermal conductivities and the base fluids include water, oil, ethylene glycol and ethanol. Significantly diverse utilization of nanofluid can be seen in modern biomedical technologies and therapies. For example, nano-drugs involve the targeted delivery of nanoparticles in cancer treatments. The anti-bacterial properties of for example silver nanoparticles have witnessed considerable popularity in sterilization techniques. Tripathi and Bég [15] performed one of the first investigations of nanoparticle doping in drug diffusion via peristaltic transport in the intestines. Tripathi et al. [16] very recently presented a detailed review on the recent advancements in nano-drug delivery systems in hemodynamics. Vasu et al. [17] conducted a comprehensive finite element analysis of magnetic body force and nanoparticle effects in electroconductive nonNewtonian blood flow in a stenosed coronary artery using FREEFEM++ software. Ali et al. [18] analyzed the unsteady heat and mass transfer in nanoparticle-doped blood flow through a tapered stenotic artery. Nadeem and Ijaz [19] employed a computational model to study metallic nanoparticle effects in stenosed blood artery including permeability effects of the vessel wall. Ahmed and Nadeem [20] explored the relative performance of various nanoparticles $\left(\mathrm{Cu}_{,} \mathrm{Al}_{2} \mathrm{O}_{3}\right.$ and $\mathrm{TiO}_{2}$ ) in blood flow through a stenosed artery. Dubey et al. [21] very recently performed numerical computations for transient non-Newtonian blood flow conveying nanoparticles through an artery with a combination of stenosis and aneurysm, providing extensive flow visualization in the vicinity of the constriction and expansion in the blood vessel.

The above investigations were restricted to a single nanoparticle type ("unitary nanofluids"). However, Makishima et al. [22] suggested nanofluid suspensions comprising of two or more 
metallic nanoparticle types suspended in base fluid to provide a homogenous phase which they termed hybrid nanofluids. In comparison to unitary nanofluid, hybrid nanofluids successfully combined the unique individual physical and chemical properties of multiple nanoparticles, which have been proven to be achieve markedly improved results. Ijaz and Nadeem [23] theoretically investigated the hybrid nanoparticle-mediated steady blood flow through an overlapped stenosed artery with tapering effects. Ijaz and Nadeem [24] simulated the stenotic arterial hemodynamics doped with hybrid (silver, copper and gold) nanoparticles, observing that higher volume fractions of gold nanoparticles achieve improved flow efficiency. Zaman et al. [25] analyzed the blood flow characteristics in a curved stenosed artery with an aneurysm, considering hybrid nanoparticles (copper, silver) and the FTCS finite difference method. Ahmed and Nadeem [26] explored the magnetohydrodynamics effects on steady non-Newtonian (micropolar) blood mediated hybrid nanoparticles $\left(\mathrm{Cu}\right.$ and $\left.\mathrm{Al}_{2} \mathrm{O}_{3}\right)$ through six different types of stenosis.

Magnetohydrodynamics (MHD) is the science studying the motion of electrically conducting fluids under the presence of applied (uniform or variable) magnetic fields. Discovered by Faraday in the $19^{\text {th }}$ century, MHD phenomena are independent of fluid rheological characteristics. In the case of blood flows, the existing ions and irons in hemoglobin give rise to electrical fluid properties. Hence applying fixed or alternating magnetic fields produces the magnetohydrodynamic effect in streaming blood. This property of blood can be exploited in for example bio-magnetic therapy to cure arterial diseases including atherosclerosis. Haik et al. [27] identified that on applying a magnetic field of 10 Tesla intensity, a reduction of $30 \%$ in the volumetric flow rate of blood is produced. Reduction in blood flow has also been confirmed by Yadav et al. [28] via applying a magnetic field of much less magnitude (0.002 Tesla) in a stenotic region. Selvi and Ponalagusamy [29] studied the impact of uniform magnetic field in blood flow through a stenosed artery with the Newtonian model, observing that an increment in magnetic field significantly elevates the blood flow resistance. Nadeem et al. [30] also analyzed the magnetohydrodynamic blood flow in a stenotic arterial region, noting again that imposition of stronger magnetic field reduces the velocity in that area.

Generally, blood viscosity is considered to be constant in most hemodynamic computations reported in the literature; however, this assumption is not practical for every situation. Viscosity is however found to be a function of temperature and pressure in the case of real fluids, including blood. Popular models utilized in this regard include the Reynolds model, Vogel model and 
Einstein's model. Zaman et al. [31] used Reynolds model to represent the viscosity of blood doped with hybrid nanoparticles in a stenosed artery. Ellahi et al. [32] used two different viscosity models (Vogel's and Reynolds' model) to determine the homotopy series solutions of nanofluid flows. Inspection of the biomedical engineering literature has revealed that, relatively few works have been performed for hemodynamic transport considering the hybrid nanoparticles featuring in overlapped stenosis region under applied magnetic field. Furthermore, relatively sparse has considered variable viscosity blood flow combined with the heat generation effects under unsteady flow conditions. Also, the alteration in blood pressure distribution in hybrid nanopharmacodynamics has not been extensively examined computationally. Consequently, the novelty of the article is the, numerical simulation of unsteady magnetohydrodynamic hybrid nanoparticle ( $\mathrm{Ag}, \mathrm{Au})$-doped variable viscosity blood flow in an overlapped stenosis region of a diseased artery with heat generation effects, which to the author's knowledge has not yet been considered in biomedical computational fluid dynamics. In this article, an axisymmetric geometry is considered under a constant radial external magnetic field. Hybrid nanoparticles (gold and silver) have been taken to represent the nano-doped blood flow in a stenosed artery. In addition to this, pulsatile pressure gradient and Reynolds viscosity model are also deployed in the model. With appropriate boundary conditions, the normalized non-linear conservation equations are solved by a stable, versatile and efficient finite difference method (FTCS i.e. forward time central space). Further simulations and visualizations (post-processing) are produced in a MATLAB symbolic software environment. The provided simulations are relevant to hybrid nano-pharmacodynamic which gives a deeper insight of the transport phenomena inherent to nanoparticle drug delivery in the treatment of cardiovascular diseases."

The current study is divided into the following subsequent sections: Section 2 illustrates the arterial geometry of the problem with associated geometric formulations. Section 3 describes the mathematical formulation of the hybrid nanofluid model for stenotic transport including conservation equations and boundary conditions. Section 4 elucidates the non-dimensionalization, which permits the introduction of key scaling parameters e.g. Hartmann (MHD) parameter. Section 5 presents a comprehensive validation of the model. Section 6 provides relevant details of the FTCS numerical technique. Section 7 presents extensive results for the effects of selected parameters on velocity, temperature, wall shear stress and flow rate in addition to streamline 
distributions (contour plots). Finally, in Section 8 the principal findings of the study are summarized and future possible pathways in nano-pharmacodynamics are identified.

\section{Arterial geometry for stenotic nano-pharmacodynamics flow}

Blood flow in this study, is considered to be, axisymmetric, laminar, incompressible and unsteady in nature. The mathematical equations representing the stenotic geometry are formulated in cylindrical co-ordinate system $(\bar{r}, \theta, \bar{z})$, in which the $\bar{r}$ variable denotes the radial coordinate, $\theta$ is a circumferential (azimuthal) coordinate and $\bar{z}$ is axial (longitudinal) co-ordinate, respectively. Since the flow is considered as axisymmetric, hence the circumferential direction is neglected and quasi-3-dimensional flow may be considered in two spatial variables $(\bar{r}, \bar{z})$. Further, the mathematical equation describing the overlapped stenosis [33] geometry in an artery is taken as:

$$
R(z)=\left\{\begin{array}{ll}
a\left[1-\frac{64}{10} \eta\left(\frac{11}{32} l_{o}^{3}(z-d)-\frac{47}{48} l_{o}^{2}(z-d)^{2}+l_{o}(z-d)^{3}-\frac{1}{3}(z-d)^{4}\right)\right], & d \leq z \leq d+\frac{3}{2} l_{o} \\
a, & \text { otherwise }
\end{array}\right\} .
$$

Here $R(z)$ defines the radius of the overlapped stenotic region, $a$ is the radius of the artery in the non-stenotic region, $d$ is the location of stenosis from origin and $l_{o}$ is the length of stenotic region. A schematic diagram of the diseased arterial segment is given in Fig.1.

The geometric parameter $\eta$ is defined as [33]:

$\eta=\frac{4 \delta^{*}}{a l_{o}^{4}}, \quad$ where $\delta=\frac{\delta^{*}}{a}$

Here $\delta^{*}$ shows the critical height of stenosis [33], which is given at two specific locations, i.e.

$$
z=d+\frac{8}{25} l_{o} \quad \text { and } \quad z=d+\frac{61}{50} l_{o}
$$




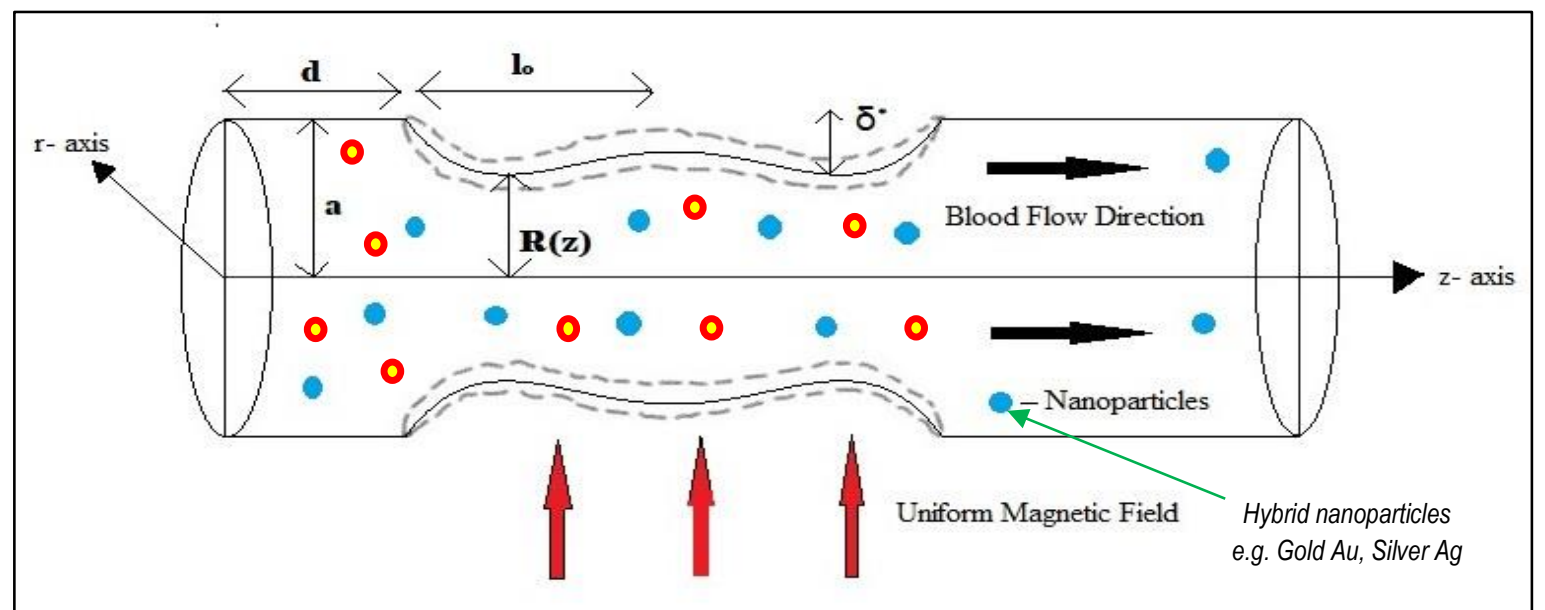

Figure 1: Geometry of the stenosed artery in which $R(z)$ is radius of the artery, $a$ is the radius in non-stenotic region, $l_{0}$ and $\delta$ shows the length and depth of the stenosis respectively and $d$ is the distance of stenosis from origin.

\section{Mathematical transport model for hybrid nanoparticle-doped blood:}

In the case of unsteady and axisymmetric hybrid nanoparticle-doped blood flow, the velocity and temperature fields for the current problem are defined as:

$V=[v(r, z, t), 0, w(r, z, t)]$

$T=T(r, z, t)$

where $v$ and $w$ are the radial and axial velocity components respectively. In view of Eqn. (4) and considering the hybrid nanoparticles model, the transport equations i.e. continuity, momentum, and energy conservation with thermal buoyancy, heat generation (source), MHD and exponential viscosity variation, can be written as [31]:

$\frac{\partial v}{\partial r}+\frac{v}{r}+\frac{\partial w}{\partial z}=0$

$\rho_{h n f}\left[\frac{\partial v}{\partial t}+v \frac{\partial v}{\partial r}+w \frac{\partial v}{\partial z}\right]=-\frac{\partial p}{\partial r}+\frac{1}{r} \frac{\partial}{\partial r}\left[\mu_{h n f}(T) \frac{\partial v}{\partial r}\right]+\frac{\partial}{\partial z}\left[\mu_{h n f}(T)\left(\frac{\partial v}{\partial z}+\frac{\partial w}{\partial r}\right)\right]-2 \mu_{h n f}(T) \frac{v}{r^{2}}$

$\rho_{h n f}\left[\frac{\partial w}{\partial t}+v \frac{\partial w}{\partial r}+w \frac{\partial w}{\partial z}\right]=-\frac{\partial p}{\partial z}+\frac{1}{r} \frac{\partial}{\partial r}\left[r \mu_{h n f}(T)\left(\frac{\partial v}{\partial z}+\frac{\partial w}{\partial r}\right)\right]+\frac{\partial}{\partial z}\left[2 \mu_{h n f}(T) \frac{\partial w}{\partial z}\right]$

$+(\rho \gamma)_{h n f} g\left(T-T_{1}\right)-\sigma_{h n f} B_{o}^{2}(w)$ 
$\left[\frac{\partial T}{\partial t}+v \frac{\partial T}{\partial r}+w \frac{\partial T}{\partial z}\right]=\frac{k_{h n f}}{\left(\rho C_{p}\right)_{h n f}}\left[\frac{\partial^{2} T}{\partial r^{2}}+\frac{1}{r} \frac{\partial T}{\partial r}+\frac{\partial^{2} T}{\partial z^{2}}\right]+\frac{\beta_{o}}{\left(\rho C_{p}\right)_{h n f}}$

The penultimate terms in Eqn. (7) denote thermal buoyancy and magnetic body force, respectively. The final term in Eqn. (8) signifies heat generation. The physical properties featured in the above set of equations are defined as follows: $\rho_{h n f}$ is the density, $\mu_{h n f}$ is the viscosity, $\gamma_{h n f}$ is the thermal expansion coefficient, $\sigma_{h n f}$ is electrical conductivity of hybrid nanofluid (blood), $k_{h n f}$ is thermal conductivity, $\left(C_{p}\right)_{h n f}$ is the specific heat at constant pressure for the hybrid nanofluid model, $B_{o}$ designates uniform radial magnetic field and $\beta_{o}$ is the heat generation parameter. For the velocity profile and temperature field, the associated boundary and initial conditions are prescribed as follows which include a hydrodynamic slip velocity at the vessel wall $\left(w_{\mathrm{s}}\right)$ :

Boundary Conditions: $\left.\quad \frac{\partial w}{\partial r}\right|_{r=0}=0,\left.\quad w\right|_{r=R}=w_{s},\left.\frac{\partial T}{\partial r}\right|_{r=0}=0,\left.\quad T\right|_{r=R}=1$

Initial Conditions: $\left.\quad w\right|_{t=0}=0,\left.\quad T\right|_{t=0}=0$

\section{Non-dimensionalization of hybrid nano-fluid transport model}

To facilitate a numerical solution and simultaneously permit the hydrodynamic scaling of the flow regime, the mathematical model defined by Eqns. (5-8) can be made dimensionless with the help of following set of parameters [31]:

$$
\begin{aligned}
& \bar{r}=\frac{r}{a}, \bar{w}=\frac{w}{U_{o}}, \quad \bar{z}=\frac{z}{l_{o}}, \bar{R}=\frac{R}{a}, \quad \bar{v}=\frac{l_{o} v}{\delta^{*} U_{o}}, \bar{t}=\frac{U_{o}}{a} t, \quad \bar{p}=\frac{a^{2} p}{U_{o} l_{o} \mu_{o}}, \theta=\frac{T-T_{1}}{T_{w}-T_{1}}, \beta=\frac{a^{2} \beta_{o}}{\left(T_{w}-T_{1}\right) k_{f}}, \\
& \operatorname{Pr}=\frac{C_{p} \mu_{f}}{k_{f}}, G r=\frac{\rho_{f} g \gamma_{f} a^{2}\left(T_{w}-T_{1}\right)}{\mu_{f} U_{o}}, \operatorname{Re}=\frac{\rho_{f} U_{o} a}{\mu_{f}}, \overline{w_{s}}=\frac{w_{s}}{U_{o}}, M=\sqrt{\frac{\sigma_{f}}{\mu_{o}}} B_{0} a
\end{aligned}
$$

Here $\bar{r}$ represents the dimensionless radial coordinate, $\bar{w}$ is the dimensionless axial velocity, $\bar{R}$ is dimensionless radius, $\bar{z}$ is dimensionless axial coordinate, $\bar{v}$ is dimensionless radial velocity component, $\bar{t}$ is dimensionless time, $\bar{p}$ is dimensionless pressure, $\theta$ is dimensionless blood temperature, $\beta$ is dimensionless heat generation parameter and $\overline{w_{s}}$ is dimensionless slip velocity. In addition to this, $P r$ is Prandtl number, $R e$ is Reynolds number, $G r$ is local thermal Grashof 
number and $M$ is magnetic interaction parameter (Hartmann number). $U_{o}$ is reference blood flow velocity and $T_{w}$ is the vessel wall temperature. In the above-defined variables, the non-dimensional geometric parameters are stenosis height parameter $\left(\delta=\delta^{*} / a\right)$ and vessel aspect ratio $\left(\varepsilon=a / l_{o}\right)$. Introducing the transformation variables and after dropping the bars, the conservation equations become:

$$
\begin{aligned}
& \delta\left[\frac{\partial v}{\partial r}+\frac{v}{r}\right]+\frac{\partial w}{\partial z}=0 \\
& \operatorname{Re}\left(\frac{\rho_{h n f}}{\rho_{f}}\right) \delta \varepsilon^{2}\left[\frac{\partial v}{\partial t}+(\delta \varepsilon) v \frac{\partial v}{\partial r}+\varepsilon w \frac{\partial v}{\partial z}\right]=-\frac{\partial p}{\partial r}+\left(\frac{\delta a}{l_{o}^{2} \mu_{o}}\right) \frac{1}{r} \frac{\partial}{\partial r}\left[\mu_{h n f}(\theta) \frac{\partial v}{\partial r}\right]+ \\
& \frac{\varepsilon^{2} a}{\mu_{o}} \frac{\partial}{\partial z}\left[\mu_{h n f}(\theta)\left(\frac{\delta \varepsilon}{l_{o}} \frac{\partial v}{\partial z}+\frac{1}{a} \frac{\partial w}{\partial r}\right)\right]-2\left(\frac{\delta \varepsilon^{2}}{\mu_{o}}\right) \mu_{h n f}(\theta)\left(\frac{v}{r^{2}}\right) \\
& \operatorname{Re}\left(\frac{\rho_{h n f}}{\rho_{f}}\right)\left[\frac{\partial w}{\partial t}+(\delta \varepsilon) v \frac{\partial w}{\partial r}+\varepsilon w \frac{\partial w}{\partial z}\right]=-\frac{\partial p}{\partial z}+\frac{1}{r} \frac{\partial}{\partial r}\left[r\left(\frac{\mu_{h n f}(\theta)}{\mu_{o}}\right)\left(\delta \varepsilon^{2} \frac{\partial v}{\partial z}+\frac{\partial w}{\partial r}\right)\right]+ \\
& \left.\frac{\left(\rho C_{p}\right)_{h n f} \frac{\partial}{\partial z}}{\left(\rho C_{p}\right)_{f}}\left(\frac{k_{f}}{k_{h n f}}\right) \operatorname{Pr} \operatorname{Re}\left[\frac{\mu_{h n f}(\theta)}{\mu_{o}}\right) \frac{\partial w}{\partial z}+(\delta \varepsilon) v \frac{\partial \theta}{\partial r}+\varepsilon w \frac{\partial \theta}{\partial z}\right]=\left[\frac{\partial^{2} \theta}{\partial r^{2}}+\frac{1}{r} \frac{\partial \theta}{\partial r}+\varepsilon^{2} \frac{\partial^{2} \theta}{\partial z^{2}}\right]+\frac{k_{f}}{k_{h n f}} \beta
\end{aligned}
$$

To simulate variable viscosity of the hybrid nanofluid, Reynolds' exponential viscosity model [32] is considered here, and the viscosity is given as:

$\mu_{f}(\theta)=\mu_{o} e^{-\eta_{o} \theta}=\mu_{o}\left[1-\eta_{o} \theta\right]$ where $\eta_{o} \ll 1$

Where the parameter $\eta_{o}$ is a viscosity constant. The equations for the thermophysical parameters of unitary nanofluid are defined following Devi and Devi [34]:

$$
\begin{array}{ll}
\mu_{n f}(\theta)=\frac{\mu_{f}(\theta)}{(1-\phi)^{5 / 2}}, & \rho_{n f}=(1-\phi) \rho_{f}+\phi \rho_{s} \\
\left(\rho C_{p}\right)_{n f}=(1-\phi)\left(\rho C_{p}\right)_{f}+\phi\left(\rho C_{p}\right)_{s}, & (\rho \gamma)_{n f}=(1-\phi)(\rho \gamma)_{f}+\phi(\rho \gamma)_{s} \\
\frac{\sigma_{n f}}{\sigma_{f}}=\frac{\sigma_{s}+2 \sigma_{f}-2 \phi\left(\sigma_{f}-\sigma_{s}\right)}{\sigma_{s}+2 \sigma_{f}+\phi\left(\sigma_{f}-\sigma_{s}\right)}, & \frac{k_{n f}}{k_{f}}=\frac{k_{s}+2 k_{f}-2 \phi\left(k_{f}-k_{s}\right)}{k_{s}+2 k_{f}+\phi\left(k_{f}-k_{s}\right)}
\end{array}
$$


Additionally, the equations for thermo-physical properties of hybrid nanofluid (dual nanoparticledoped blood) are [34]:

$$
\begin{aligned}
& \mu_{h n f}=\frac{\mu_{f}(\theta)}{\left(1-\phi_{1}\right)^{5 / 2}\left(1-\phi_{2}\right)^{5 / 2}}, \quad \rho_{h n f}=\left(1-\phi_{2}\right)\left[\left(1-\phi_{1}\right) \rho_{f}+\phi_{1} \rho_{S_{1}}\right]+\phi_{2} \rho_{S_{2}} \\
& \left(\rho C_{p}\right)_{h n f}=\left(1-\phi_{2}\right)\left[\left(1-\phi_{1}\right)\left(\rho C_{p}\right)_{f}+\phi_{1}\left(\rho C_{p}\right)_{S_{1}}\right]+\phi_{2}\left(\rho C_{p}\right)_{S_{2}} \\
& (\rho \gamma)_{h n f}=\left(1-\phi_{2}\right)\left[\left(1-\phi_{1}\right)(\rho \gamma)_{f}+\phi_{1}(\rho \gamma)_{S_{1}}\right]+\phi_{2}(\rho \gamma)_{S_{2}} \\
& \frac{\sigma_{h n f}}{\sigma_{b f}}=\frac{\sigma_{S_{2}}+2 \sigma_{b f}-2 \phi_{2}\left(\sigma_{b f}-\sigma_{S_{2}}\right)}{\sigma_{S_{2}}+2 \sigma_{b f}+\phi_{2}\left(\sigma_{b f}-\sigma_{S_{2}}\right)}, \quad \text { where } \frac{\sigma_{b f}}{\sigma_{f}}=\frac{\sigma_{S_{1}}+2 \sigma_{f}-2 \phi_{1}\left(\sigma_{f}-\sigma_{S_{1}}\right)}{\sigma_{S_{1}}+2 \sigma_{f}+\phi_{1}\left(\sigma_{f}-\sigma_{S_{1}}\right)} \\
& \frac{k_{h n f}}{k_{b f}}=\frac{k_{S_{2}}+2 k_{b f}-2 \phi_{2}\left(k_{b f}-k_{S_{2}}\right)}{k_{S_{2}}+2 k_{b f}+\phi_{2}\left(k_{b f}-k_{S_{2}}\right)}, \quad \text { where } \frac{k_{b f}}{k_{f}}=\frac{k_{S_{1}}+2 k_{f}-2 \phi_{1}\left(k_{f}-k_{S_{1}}\right)}{k_{S_{1}}+2 k_{f}+\phi_{1}\left(k_{f}-k_{S_{1}}\right)}
\end{aligned}
$$

In the above-mentioned equations, $\mu_{f}$ is the viscosity of the base fluid, $\rho_{f}$ is base fluid density, $\left(\rho C_{p}\right)_{f}$ is base fluid heat capacitance, $\gamma_{f}$ is thermal expansion coefficient, $\sigma_{f}$ as electrical conductivity and $k_{f}$ is the thermal conductivity of the base fluid. For different nanoparticles, $\left(\phi_{1}, \phi_{2}\right)$ denote the individual volume fractions, $\left(\rho_{S_{1}}, \rho_{S_{2}}\right)$ are the densities of nanoparticles,

\begin{tabular}{|c|c|c|c|c|c|c|}
\hline \multirow{2}{*}{$\begin{array}{l}\text { Thermophysical } \\
\text { properties }\end{array}$} & \multirow{2}{*}{$\begin{array}{l}\text { Blood } \\
(f)\end{array}$} & \multicolumn{5}{|c|}{ Solid metallic/ metallic oxide nanoparticles ( $s$ ) } \\
\hline & & $(\mathrm{Ag})$ & (Au) & $(\mathbf{C u})$ & $\left(\mathrm{TiO}_{2}\right)$ & $\left(\mathrm{Al}_{\mathbf{2}} \mathrm{O}_{3}\right)$ \\
\hline Density $\left[\rho\left(k g / m^{3}\right)\right]$ & 1063 & 10500 & 19320 & 8933 & 4250 & 3970 \\
\hline $\begin{array}{l}\text { Thermal Conductivity } \\
{[(W / m K)]}\end{array}$ & 0.492 & 429 & 314 & 400 & 8.9538 & 40 \\
\hline \begin{tabular}{llr} 
Thermal & \multicolumn{2}{c}{ expansion } \\
coefficient & {$\left[\begin{array}{ll}\gamma \mathbf{x} & 1^{-5} \\
\left.\left(K^{-1}\right)\right] & \end{array}\right.$}
\end{tabular} & 0.18 & 1.89 & 1.4 & 1.67 & 0.9 & 0.85 \\
\hline $\begin{array}{l}\text { Heat Capacitance } \\
{\left[C_{p}(J / k g K)\right]}\end{array}$ & 3594 & 235 & 129 & 385 & 686.2 & 765 \\
\hline
\end{tabular}
$\left[\left(\rho C_{p}\right)_{S_{1}},\left(\rho C_{p}\right)_{S_{2}}\right]$ are the heat capacitances, $\left[\gamma_{S_{1}}, \gamma_{S_{2}}\right]$ are thermal expansion coefficients, $\left[\sigma_{S_{1}}, \sigma_{S_{2}}\right]$ signify electrical conductivities, and $\left[k_{S_{1}}, k_{S_{2}}\right]$ denote thermal conductivities of solid nanoparticles, respectively. Hydrodynamic, thermal and magnetic properties for 5 different nanoparticles and blood are given below in Table 1. 


\begin{tabular}{|l|l|l|l|l|l|l|}
\hline $\begin{array}{l}\text { Electrical Conductivity } \\
{[\sigma(S / m)]}\end{array}$ & $6.67 \times 10^{-1}$ & $6.3 \times 10^{7}$ & $4.52 \times 10^{7}$ & $5.96 \times 10^{7}$ & $2.38 \times 10^{6}$ & $3.5 \times 10^{7}$ \\
\hline
\end{tabular}

Table 1: Thermophysical properties of blood and various nanoparticles

In reference to further analysis, two different hypotheses have been taken into account; $\delta \ll 1$ and $\varepsilon=O(1)$ i.e. stenosis height parameter is much less than one, and the vessel aspect ratio is of comparable magnitude to unity. After imposing these suppositions, Eqns. (12)-(15) therefore contract to:

$\frac{\partial w}{\partial z}=0$
$\frac{\partial p}{\partial r}=0$

$\operatorname{Re}\left(\frac{\rho_{h n f}}{\rho_{f}}\right)\left[\frac{\partial w}{\partial t}\right]=-\frac{\partial p}{\partial z}+\frac{1}{r} \frac{\partial}{\partial r}\left[r\left(\frac{\mu_{h n f}(\theta)}{\mu_{o}}\right)\left(\frac{\partial w}{\partial r}\right)\right]+\frac{(\rho \gamma)_{h n f}}{(\rho \gamma)_{f}} G r \theta-\left(\frac{\sigma_{h n f}}{\sigma_{f}}\right) M^{2}(w)$

$\frac{\left(\rho C_{p}\right)_{h n f}}{\left(\rho C_{p}\right)_{f}}\left(\frac{k_{f}}{k_{h n f}}\right) \operatorname{Pr} \operatorname{Re}\left[\frac{\partial \theta}{\partial t}\right]=\left[\frac{\partial^{2} \theta}{\partial r^{2}}+\frac{1}{r} \frac{\partial \theta}{\partial r}\right]+\frac{k_{f}}{k_{h n f}} \beta$

Inserting the values of $\left(\frac{\rho_{h n f}}{\rho_{f}}\right),\left(\frac{\mu_{h n f}(\theta)}{\mu_{o}}\right),\left(\frac{(\rho \gamma)_{h n f}}{(\rho \gamma)_{f}}\right)$ and $\left(\frac{\left(\rho C_{p}\right)_{h n f}}{\left(\rho C_{p}\right)_{f}}\right)$ in the above equations, the revised equations for hybrid nanofluid (blood) emerge as:

$$
\begin{aligned}
& {\left[\left(1-\phi_{2}\right)\left[\left(1-\phi_{1}\right)+\phi_{1} \frac{\rho_{S_{1}}}{\rho_{f}}\right]+\phi_{2} \frac{\rho_{S_{2}}}{\rho_{f}}\right] \operatorname{Re}\left[\frac{\partial w}{\partial t}\right]=-\frac{\partial p}{\partial z}+\frac{\left(1-\eta_{o} \theta\right)}{\left(1-\phi_{1}\right)^{5 / 2}\left(1-\phi_{2}\right)^{5 / 2}}\left[\frac{\partial^{2} w}{\partial r^{2}}+\frac{1}{r} \frac{\partial w}{\partial r}\right]+} \\
& {\left[\left(1-\phi_{2}\right)\left[\left(1-\phi_{1}\right)+\phi_{1} \frac{(\rho \gamma)_{S_{1}}}{(\rho \gamma)_{f}}\right]+\phi_{2} \frac{(\rho \gamma)_{S_{2}}}{(\rho \gamma)_{f}}\right] G r \theta-\left(\frac{\sigma_{h n f}}{\sigma_{f}}\right) M^{2}(w)} \\
& {\left[\left(1-\phi_{2}\right)\left[\left(1-\phi_{1}\right)+\phi_{1} \frac{\left(\rho C_{p}\right)_{S_{1}}}{\left(\rho C_{p}\right)_{f}}\right]+\phi_{2} \frac{\left(\rho C_{p}\right)_{S_{2}}}{\left(\rho C_{p}\right)_{f}}\right]\left(\frac{k_{f}}{k_{h n f}}\right) \operatorname{Pr} \operatorname{Re}\left[\frac{\partial \theta}{\partial t}\right]=\left[\frac{\partial^{2} \theta}{\partial r^{2}}+\frac{1}{r} \frac{\partial \theta}{\partial r}\right]+\frac{k_{f}}{k_{h n f}} \beta}
\end{aligned}
$$


Where $\frac{k_{f}}{k_{\text {hnf }}}=\left(\frac{k_{S_{1}}+2 k_{f}+\phi_{1}\left(k_{f}-k_{S_{1}}\right)}{k_{S_{1}}+2 k_{f}-2 \phi_{1}\left(k_{f}-k_{S_{1}}\right)}\right)\left(\frac{k_{S_{2}}+2 k_{b f}+\phi_{2}\left(k_{b f}-k_{S_{2}}\right)}{k_{S_{2}}+2 k_{b f}-2 \phi_{2}\left(k_{b f}-k_{S_{2}}\right)}\right)$

and $\frac{\sigma_{h n f}}{\sigma_{f}}=\left(\frac{\sigma_{S_{2}}+2 \sigma_{b f}-2 \phi_{2}\left(\sigma_{b f}-\sigma_{S_{2}}\right)}{\sigma_{S_{2}}+2 \sigma_{b f}+\phi_{2}\left(\sigma_{b f}-\sigma_{S_{2}}\right)}\right)\left(\frac{\sigma_{S_{1}}+2 \sigma_{f}-2 \phi_{1}\left(\sigma_{f}-\sigma_{S_{1}}\right)}{\sigma_{S_{1}}+2 \sigma_{f}+\phi_{1}\left(\sigma_{f}-\sigma_{S_{1}}\right)}\right)$.

With the help of Burton [35], pulsatile hemodynamic effect is simulated via the inclusion of an oscillatory axial pressure gradient as follows:

$-\frac{\partial p}{\partial z}=A_{o}+A_{1} \cos \left(2 \pi w_{p}\right) t, t>0$

Here $A_{0}$ is the mean pressure gradient and $A_{1}$ denotes the amplitude of the pulsatile component, which is subject to diastolic and systolic pressures. By using non-dimensional parameters defined earlier in Eqn. (11), the normalized form of Eqn. (24) becomes:

$-\frac{\partial p}{\partial z}=B_{1}\left[1+e \cos \left(c_{1} t\right)\right]$

Where $e=\frac{A_{1}}{A_{o}}, \quad B_{1}=\frac{A_{o} a^{2}}{\mu_{o} U_{o}}, \quad c_{1}=\frac{2 \pi a w_{p}}{U_{o}}$

Invoking Eqn. (25), the reduced axial momentum equation, Eqn. (22) becomes:

$$
\begin{aligned}
& {\left[\left(1-\phi_{2}\right)\left[\left(1-\phi_{1}\right)+\phi_{1} \frac{\rho_{S_{1}}}{\rho_{f}}\right]+\phi_{2} \frac{\rho_{S_{2}}}{\rho_{f}}\right] \operatorname{Re}\left[\frac{\partial w}{\partial t}\right]=B_{1}\left(1+e \cos \left(c_{1} t\right)\right)+\frac{\left(1-\eta_{o} \theta\right)}{\left(1-\phi_{1}\right)^{5 / 2}\left(1-\phi_{2}\right)^{5 / 2}}\left[\frac{\partial^{2} w}{\partial r^{2}}+\frac{1}{r} \frac{\partial w}{\partial r}\right]+} \\
& {\left[\left(1-\phi_{2}\right)\left[\left(1-\phi_{1}\right)+\phi_{1} \frac{(\rho \gamma)_{S_{1}}}{(\rho \gamma)_{f}}\right]+\phi_{2} \frac{(\rho \gamma)_{S_{2}}}{(\rho \gamma)_{f}}\right] G r \theta-\left(\frac{\sigma_{h n f}}{\sigma_{f}}\right) M^{2}(w) }
\end{aligned}
$$

Further, Eqns. (23) and (27) are subject to the following boundary and initial conditions:

$$
\begin{array}{lll}
\left.w(r, t)\right|_{r=R}=w_{S}, & \left.\frac{\partial w(r, t)}{\partial r}\right|_{r=0}=0, & w(r, 0)=0 \\
\left.\theta(r, t)\right|_{r=R}=1, & \left.\frac{\partial \theta(r, t)}{\partial r}\right|_{r=0}=0, & \theta(r, 0)=0
\end{array}
$$

Key hemodynamic quantities such as volumetric flow rate and wall shear stress (WSS) may be formulated as: 


$$
\begin{aligned}
& Q_{1}=2 \pi \int_{0}^{R} w r d r \\
& \tau_{s}=-\left(\frac{\partial w}{\partial r}\right)_{r=R}
\end{aligned}
$$

With respect to the above non-dimensional parameters, the geometrical relation for the stenotic arterial region can be written in non-dimensional form as:

$$
R(z)=1-\left(\frac{64}{10}\right) \eta^{*}\left(\frac{11}{32}\left(z-\beta_{1}\right)-\frac{47}{48}\left(z-\beta_{1}\right)^{2}+\left(z-\beta_{1}\right)^{3}-\frac{1}{3}\left(z-\beta_{1}\right)^{4}\right), \quad \beta_{1} \leq z \leq \beta_{1}+\frac{3}{2}
$$

With $\eta^{*}=4 \delta, \delta=\frac{\delta^{*}}{a}, \beta_{1}=\frac{d}{l_{0}}$.

For restraining the geometric effects, the radial coordinate transformation $x=\frac{r}{R(z)}$ is employed in the above governing equations. Hence Eqns. (23), (27), (28) and (29) are reduced to the following form:

$$
\begin{aligned}
& {\left[\left(1-\phi_{2}\right)\left[\left(1-\phi_{1}\right)+\phi_{1} \frac{\rho_{S_{1}}}{\rho_{f}}\right]+\phi_{2} \frac{\rho_{S_{2}}}{\rho_{f}}\right] \operatorname{Re}\left[\frac{\partial w}{\partial t}\right]=B_{1}\left[1+e \cos \left(c_{1} t\right)\right]+\frac{\left(1-\eta_{o} \theta\right)}{\left(1-\phi_{1}\right)^{5 / 2}\left(1-\phi_{2}\right)^{5 / 2}}\left(\frac{1}{R^{2}}\right)\left[\frac{\partial^{2} w}{\partial x^{2}}+\frac{1}{x} \frac{\partial w}{\partial x}\right]+} \\
& {\left[\left(1-\phi_{2}\right)\left[\left(1-\phi_{1}\right)+\phi_{1} \frac{(\rho \gamma)_{S_{1}}}{(\rho \gamma)_{f}}\right]+\phi_{2} \frac{(\rho \gamma)_{S_{2}}}{(\rho \gamma)_{f}}\right] \operatorname{Gr} \theta-\left(\frac{\sigma_{h n f}}{\sigma_{f}}\right) M^{2}(w)} \\
& {\left[\left(1-\phi_{2}\right)\left[\left(1-\phi_{1}\right)+\phi_{1} \frac{\left(\rho C_{p}\right)_{S_{1}}}{\left(\rho C_{p}\right)_{f}}\right]+\phi_{2} \frac{\left(\rho C_{p}\right)_{S_{2}}}{\left(\rho C_{p}\right)_{f}}\right]\left(\frac{k_{f}}{k_{h n f}}\right) \operatorname{Pr} \operatorname{Re}\left[\frac{\partial \theta}{\partial t}\right]=\left(\frac{1}{R^{2}}\right)\left[\frac{\partial^{2} \theta}{\partial x^{2}}+\frac{1}{x} \frac{\partial \theta}{\partial x}\right]+\frac{k_{f}}{k_{h n f}} \beta}
\end{aligned}
$$

Furthermore, the associated dimensionless boundary conditions are written as:

$$
\begin{array}{lll}
\left.\frac{\partial w}{\partial x}\right|_{x=0}=0, & \left.w\right|_{x=1}=w_{s}, & \left.w\right|_{t=0}=0 \\
\left.\frac{\partial \theta}{\partial x}\right|_{x=0}=0, & \left.\theta\right|_{x=1}=1, & \left.\theta\right|_{t=0}=0
\end{array}
$$

Similarly, the dimensionless volumetric flow rate and wall shear stress respectively take the following form: 
$Q_{1}=2 \pi R^{2}\left(\int_{0}^{1} w x d x\right)$

$\tau_{s}=-\frac{1}{R}\left(\frac{\partial w}{\partial x}\right)_{x=1}$

\section{Numerical Solution with FTCS Scheme}

To obtain the exact solution of the transformed nonlinear partial differential equations (PDEs) is difficult, if not intractable. Hence it is necessary to use numerical methods to obtain approximate solutions for the nonlinear, coupled Eqns. (33)- (34). For the solution of these equations, an appropriate computational procedure based on the discretization of PDEs is an explicit finitedifference technique, the FTCS algorithm (forward in time (FT) and central in space (CS)) which is described in some detail by Hoffmann [36]. FTCS is quite versatile and easy to program and has therefore been applied in a number of hemodynamic and magnetohydrodynamic studies - see [25] and [31]. In this scheme, firstly the spatial domain is discretized and next the value of velocity component is calculated at each node $x_{i}$ and over the time instant $t^{k}$, which is written as $w_{i}^{k}$. According to Hoffmann [36], the central differencing formulation for second-order partial derivatives and the forward differencing formulation for first-order partial derivatives are defined by the following discretization formulae:

$$
\begin{aligned}
& \frac{\partial w}{\partial x} \cong \frac{w_{i+1}^{k}-w_{i-1}^{k}}{2 \Delta x}=w_{x}, \\
& \frac{\partial^{2} w}{\partial x^{2}} \cong \frac{w_{i+1}^{k}-2 w_{i}^{k}+w_{i-1}^{k}}{(\Delta x)^{2}}=w_{x x}, \\
& \frac{\partial w}{\partial t} \cong \frac{w_{i}^{k+1}-w_{i}^{k}}{(\Delta t)}
\end{aligned}
$$

Incorporating the relevant discretized partial derivatives, Eqns. (33)-(34) will readily be reduced to the forms given below:

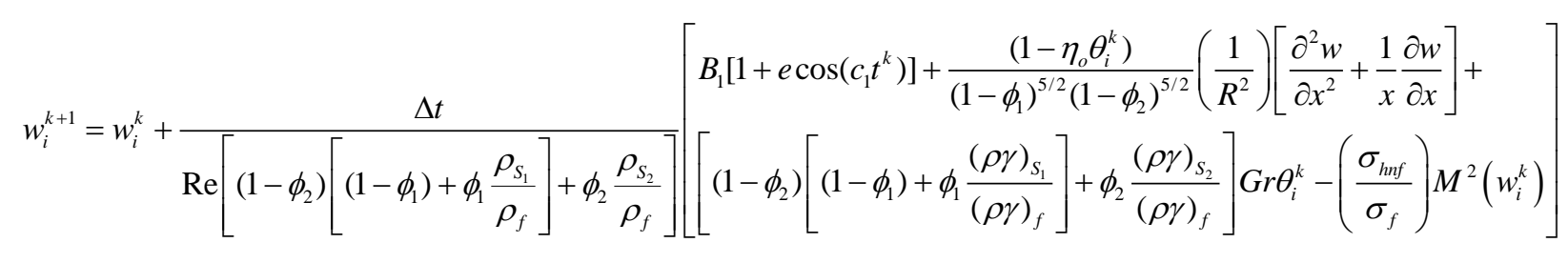




$$
\theta_{i}^{k+1}=\theta_{i}^{k}+\left(\frac{k_{h n f}}{k_{f}}\right) \frac{\Delta t}{\operatorname{Pr} \operatorname{Re}\left[\left(1-\phi_{2}\right)\left[\left(1-\phi_{1}\right)+\phi_{1} \frac{\left(\rho C_{p}\right)_{S_{1}}}{\left(\rho C_{p}\right)_{f}}\right]+\phi_{2} \frac{\left(\rho C_{p}\right)_{S_{2}}}{\left(\rho C_{p}\right)_{f}}\right]}\left\{\left(\frac{1}{R^{2}}\right)\left[\frac{\partial^{2} \theta}{\partial x^{2}}+\frac{1}{x} \frac{\partial \theta}{\partial x}\right]+\frac{k_{f}}{k_{h n f}} \beta\right\}
$$

Further, the associated boundary and initial conditions are designated:

$$
\begin{array}{ll}
w_{i}^{1}=\theta_{i}^{1}=0, & \text { at } t=0 \\
w_{i+1}^{k}=w_{i}^{k}, \theta_{i+1}^{k}=\theta_{i}^{k}, & \text { at } x=0 \\
w_{N+1}^{k}=w_{s}, \theta_{N+1}^{k}=1, & \text { at } x=1
\end{array}
$$

In FTCS computations, the spatial variable is discretized into $N+1$ grid-points where the step size

is taken as $\Delta x=1 / N+1$. The time instant is defined by $t_{k}$, where the value of $t_{k}$ is given as $t_{k}=(k-1) \Delta t$, in which $\Delta t$ denotes a small increment in time. The velocity component is further calculated at each node and for every time instant. As stability of this numerical scheme is dependent on step size and time increment, after mesh testing and trial simulations, $\Delta x=0.025$ and $\Delta t=0.0001$ are selected to fulfill the stability condition. These values confirm the stability and convergence of this scheme as verified in Hoffmann [36]. The FTCS code has been extensively validated and implemented in numerous previous simulations including solar energy radiative heat transfer flows [37], oscillatory hydromagnetic flows in geophysics (MHD-assisted oil spill cleanup) [38], viscoelastic blood flow with body acceleration [39], peristaltic gastro-intestinal pumping [40], thermosolutal Cross fluid rheo-hemodynamics in tapered arteries [41] and non-Newtonian coating hydromechanics [42]. FTCS is therefore very adaptable and offers excellent accuracy in magneto-nano-hemodynamics simulation, as confirmed in the next section.

\section{Validation of FTCS numerical scheme}

To validate the FTCS numerical code which has been chosen to solve the current problem, the numerical results obtained by Zaman et al. [31] for the axial blood velocity were compared with the present computations. The comparison is documented in Table 2 for both no slip $\left(w_{\mathrm{s}}=0\right)$ and slip conditions at the arterial wall $\left(w_{\mathrm{s}}=0.1\right)$ at the throat of stenotic artery $\mathrm{z}=0.71$ and $t=1.15$. In 
both cases there was strong flow deceleration computed with increasing radial coordinate $(r)$. Evidently very close correlation is achieved and confidence in the present FTCS scheme is therefore justifiably high.

\begin{tabular}{|c|c|c|c|c|}
\hline$r$ & $\begin{array}{c}\text { Zaman et al. } \\
{[31]} \\
w_{S}=0.0\end{array}$ & $\begin{array}{c}\text { Present Results } \\
w_{S}=0.0\end{array}$ & $\begin{array}{c}\text { Zaman et al. } \\
\qquad w_{S}=0.1\end{array}$ & $\begin{array}{c}\text { Present Results } \\
w_{S}=0.1\end{array}$ \\
\hline 0 & 0.5859 & 0.5855 & 0.6857 & 0.6807 \\
\hline 0.10 & 0.5829 & 0.5819 & 0.6827 & 0.6772 \\
\hline 0.20 & 0.5725 & 0.5698 & 0.6723 & 0.6653 \\
\hline 0.30 & 0.5540 & 0.5490 & 0.6539 & 0.6448 \\
\hline 0.40 & 0.5261 & 0.5187 & 0.6259 & 0.6149 \\
\hline 0.50 & 0.4864 & 0.4773 & 0.5863 & 0.5741 \\
\hline 0.60 & 0.4323 & 0.4228 & 0.5322 & 0.5202 \\
\hline 0.70 & 0.3605 & 0.3521 & 0.4604 & 0.4502 \\
\hline 0.80 & 0.2671 & 0.2613 & 0.3671 & 0.3600 \\
\hline 0.90 & 0.1483 & 0.1457 & 0.2483 & 0.2451 \\
\hline 1.00 & 0 & 0 & 0.1000 & 0.1000 \\
\hline
\end{tabular}

Table 2: Comparison of results with Zaman et al. [31] for axial velocity results at throat of stenotic artery $\mathrm{z}=0.71$ and $t=1.15$

\section{Computational results and discussion}

In this section, the modification in hemodynamic characteristics in the overlapped stenotic artery in the presence of hybrid nanoparticles is presented. With reference to emerging parameters, the results for axial velocity, temperature profile, wall shear stress, flow rate and pressure gradient are visualized graphically in Figs. 2-32, which also additionally show the influence of hybrid nanofluid $(\mathrm{Ag}-\mathrm{Au} / \mathrm{Blood})$ in comparison to unitary nanofluid $(\mathrm{Ag} / \mathrm{Blood})$ and an impact of magnetic field on blood flow via several contour plots. Table 3 presents the default values for the key parameters implemented in the FTCS computations. 


\begin{tabular}{|l|l|l|l|l|l|l|l|l|l|l|l|}
\hline Parameter & $\phi_{1}$ & $\phi_{2}$ & $B_{1}$ & $c_{1}$ & $\eta_{0}$ & $G r$ & $\operatorname{Pr}$ & $\operatorname{Re}$ & $M$ & $\beta$ & $w_{S}$ \\
\hline Value & 0.03 & 0.03 & 1.41 & 1 & 0.2 & 0.1 & 14 & 2 & 1 & 0.1 & 0.1 \\
\hline
\end{tabular}

Table 3: Default values of key parameters used in simulations
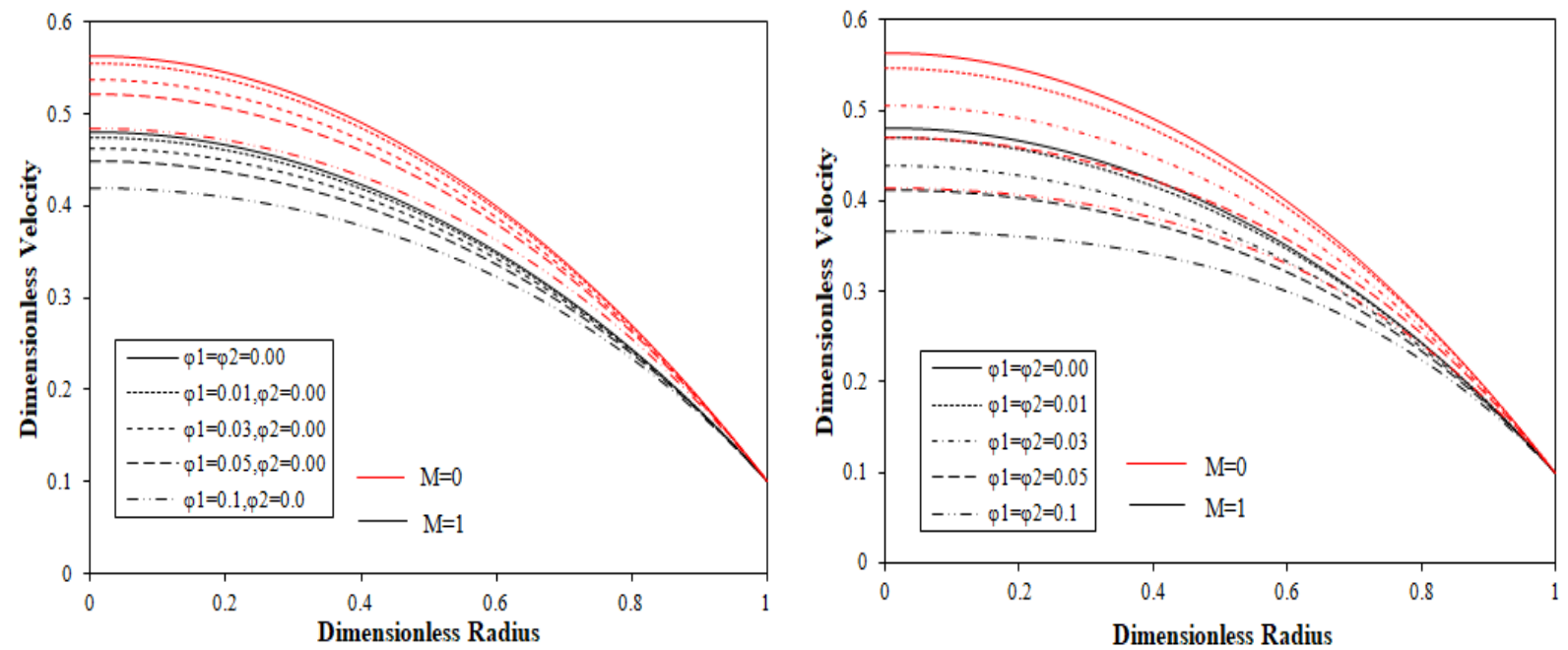

Figure 2: Effect of nanoparticle concentration on axial velocity with (a) Ag nanofluid only (b) Ag-Au hybrid nanofluid for with and without magnetic field and $B_{1}=1.41, \delta=0.1, z=0.79, t=1.2$.
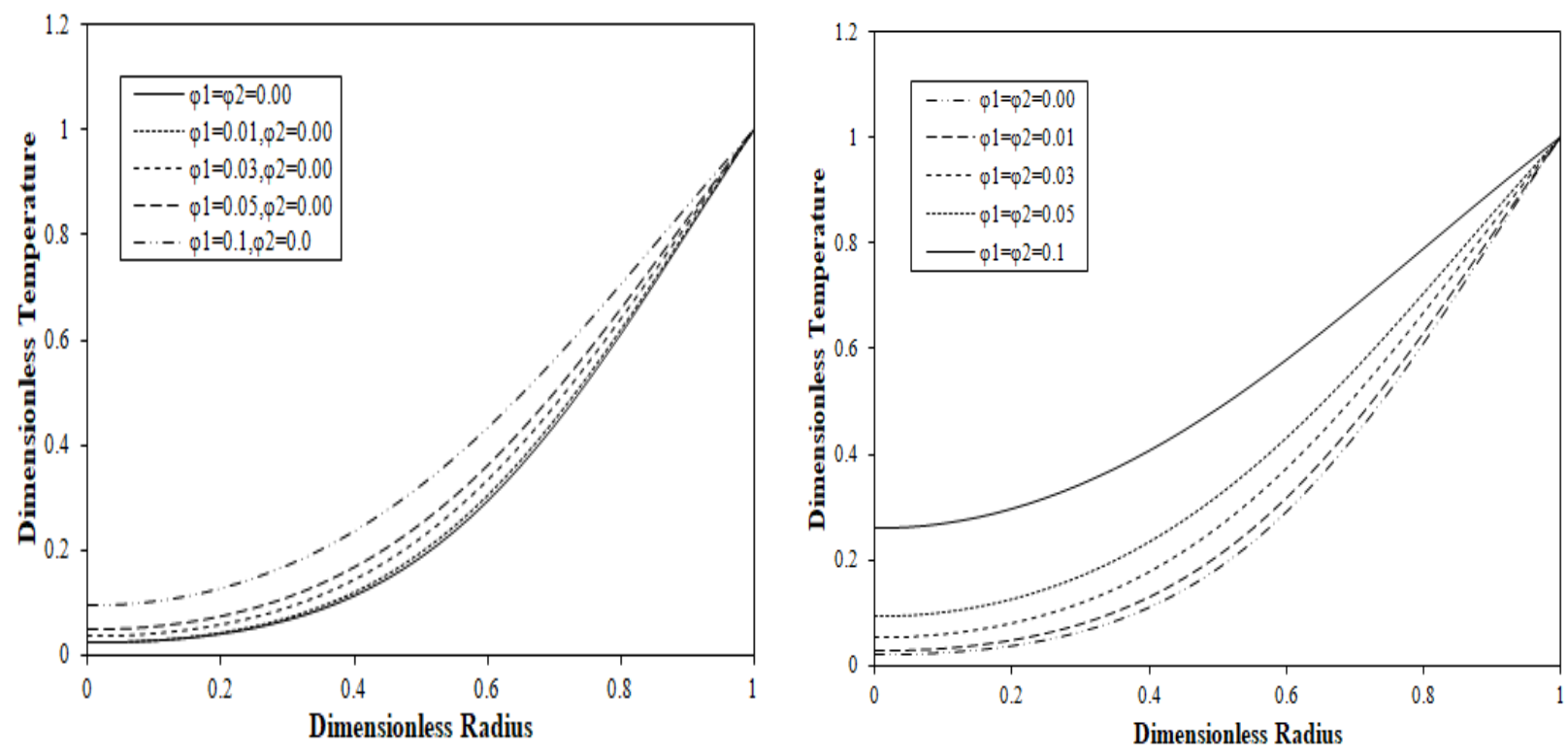

Figure 3: Effect of nanoparticle concentration on temperature profile with (a) Ag nanofluid only (b) Ag-Au hybrid nanofluid for $B_{1}=1.41, \delta=0.1, z=0.79, t=1.2$ 

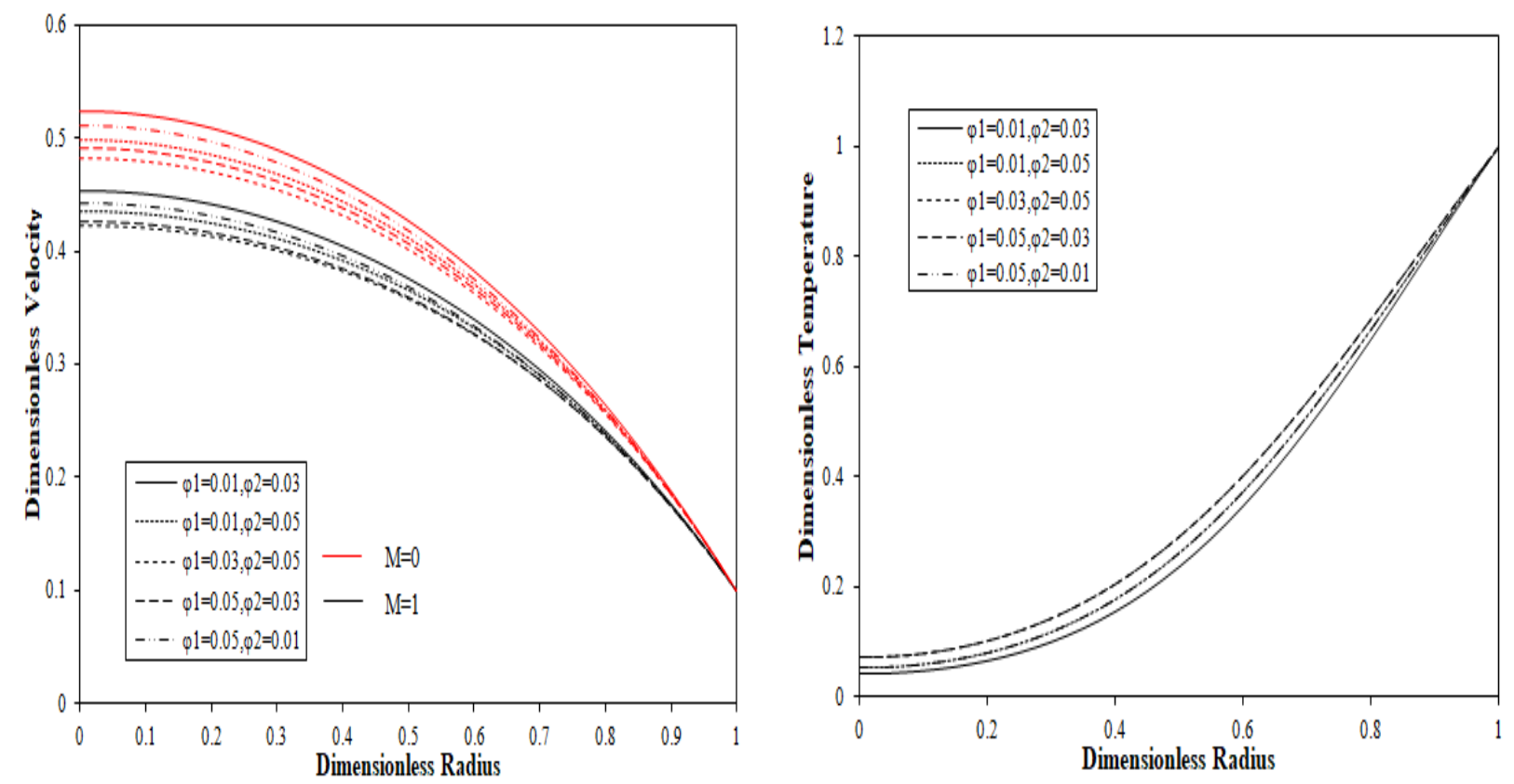

Figure 4: Effect of different nanoparticle concentration combinations on $(a)$ velocity and $(b)$ temperature profile for with and without magnetic field and with $B_{1}=1.41, \delta=0.1, z=0.79, t=1.2$.
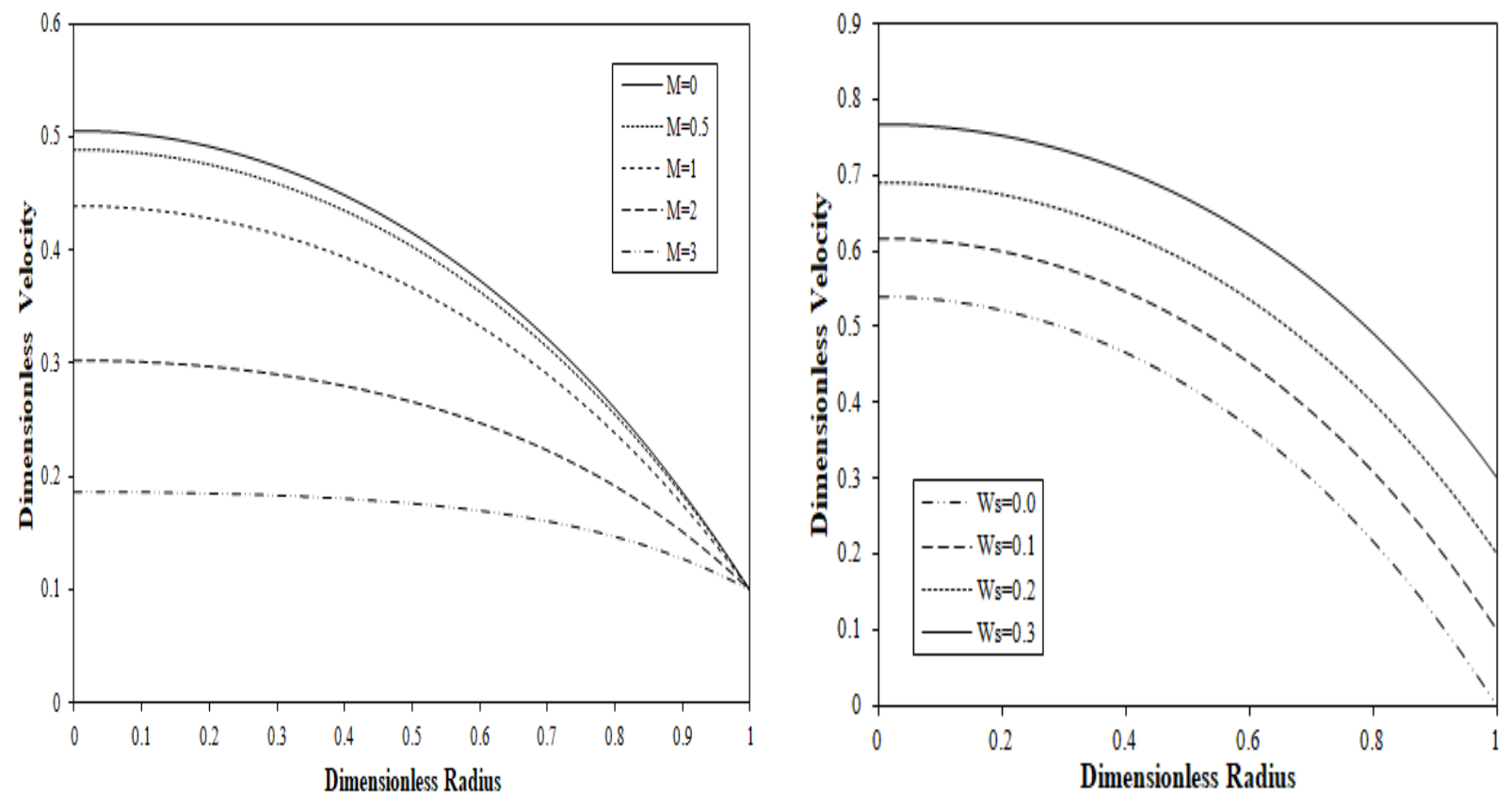

Figure 5: Effect on axial velocity for various (a) Magnetic number and (b) slip velocity parameter with $B_{1}=$ $1.41, \delta=0.1, z=0.79, t=1.2$. 

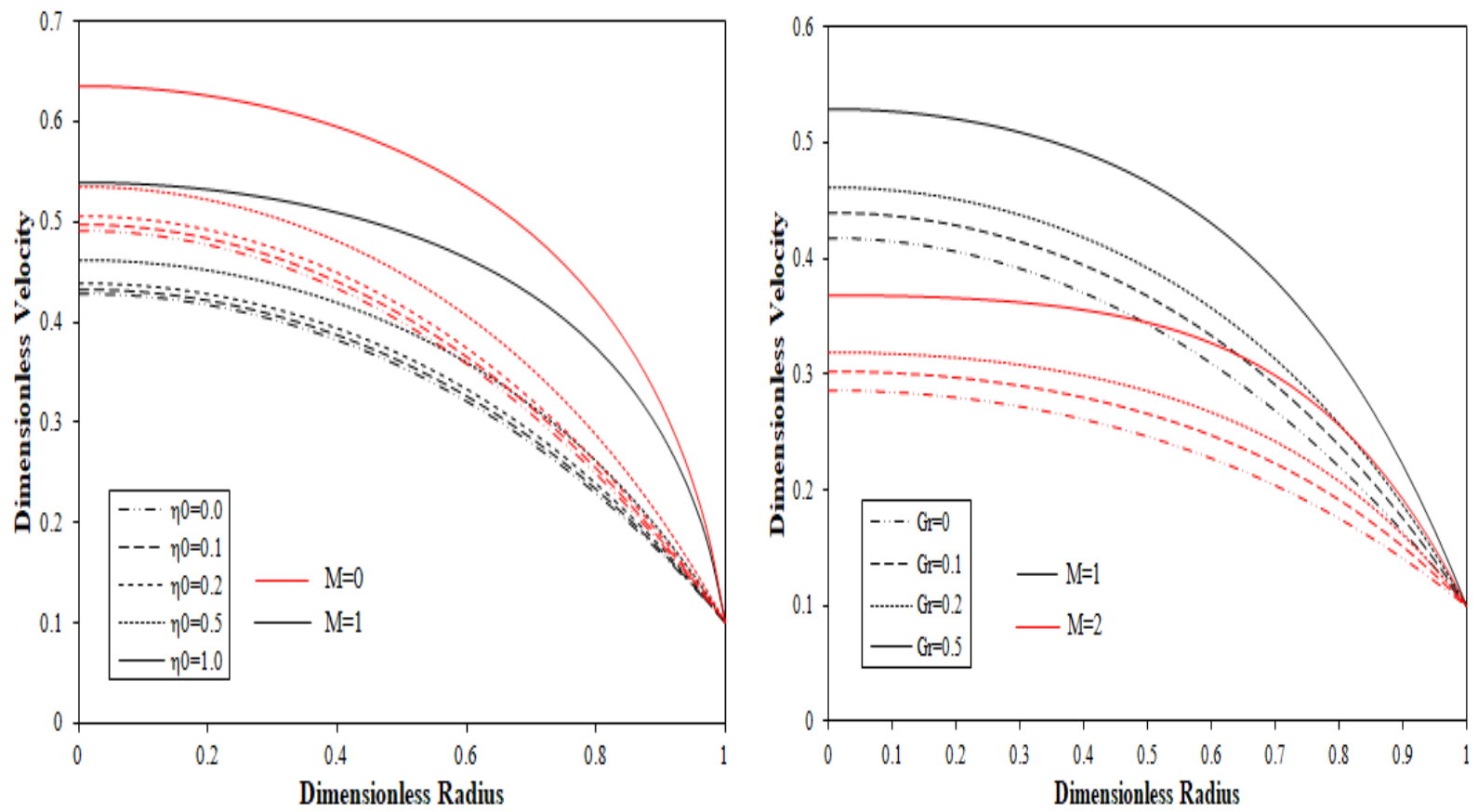

Figure 6: Effect of (a) $\eta_{0}$ and (b) Grashof number on axial velocity for with and without magnetic field and with $B_{1}=1.41, \delta=0.1, z=0.79, t=1.2$.
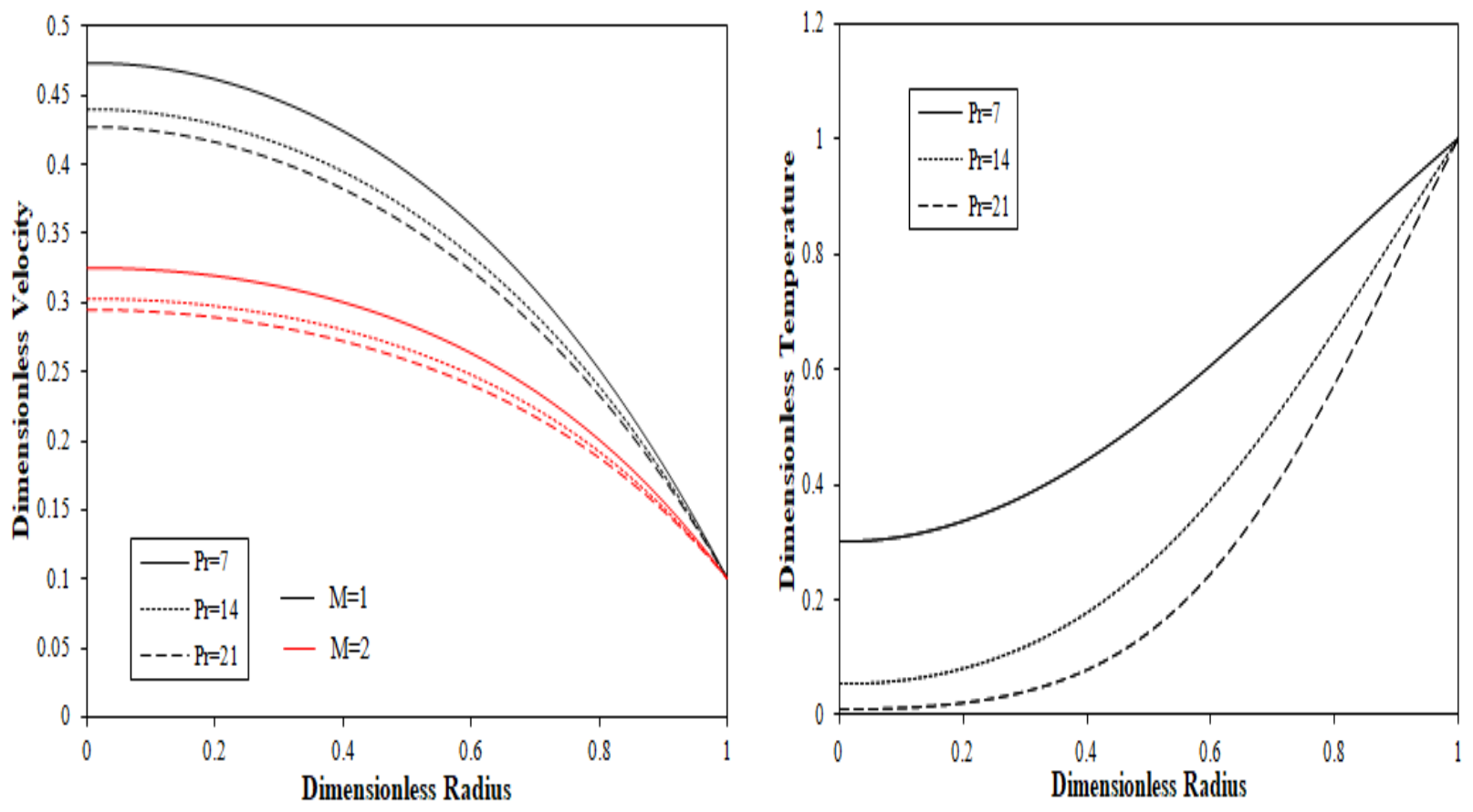

Figure 7: Effect of Prandtl number $(P r)$ on $(a)$ velocity and (b) temperature profile for $B_{1}=1.41, \delta=0.1, z=$ $0.79, t=1.2$. 

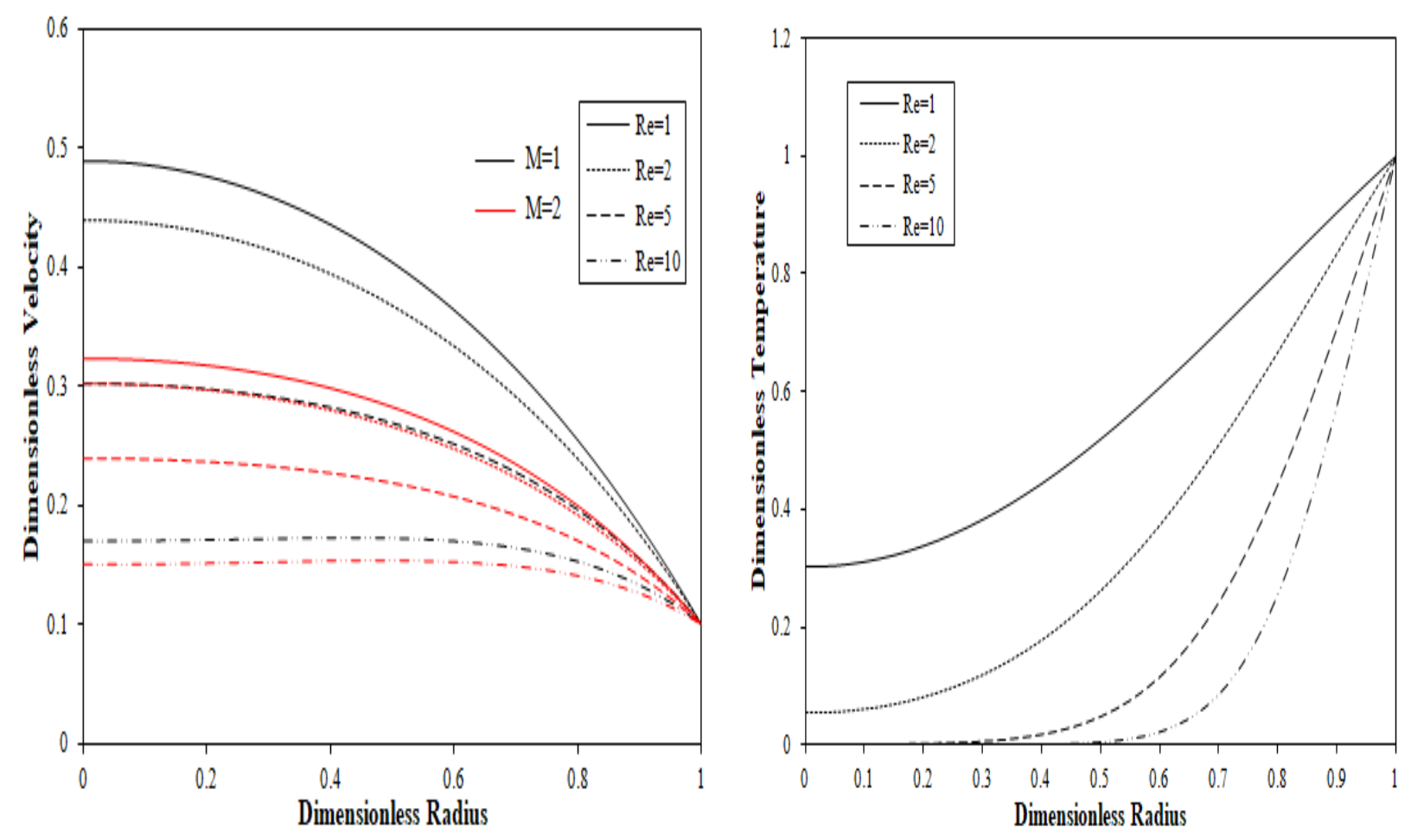

Figure 8: Effect of Reynolds number (Re) on (a) velocity and (b) temperature profile for $B_{1}=1.41, \delta=0.1, z=$ $0.79, t=1.2$.
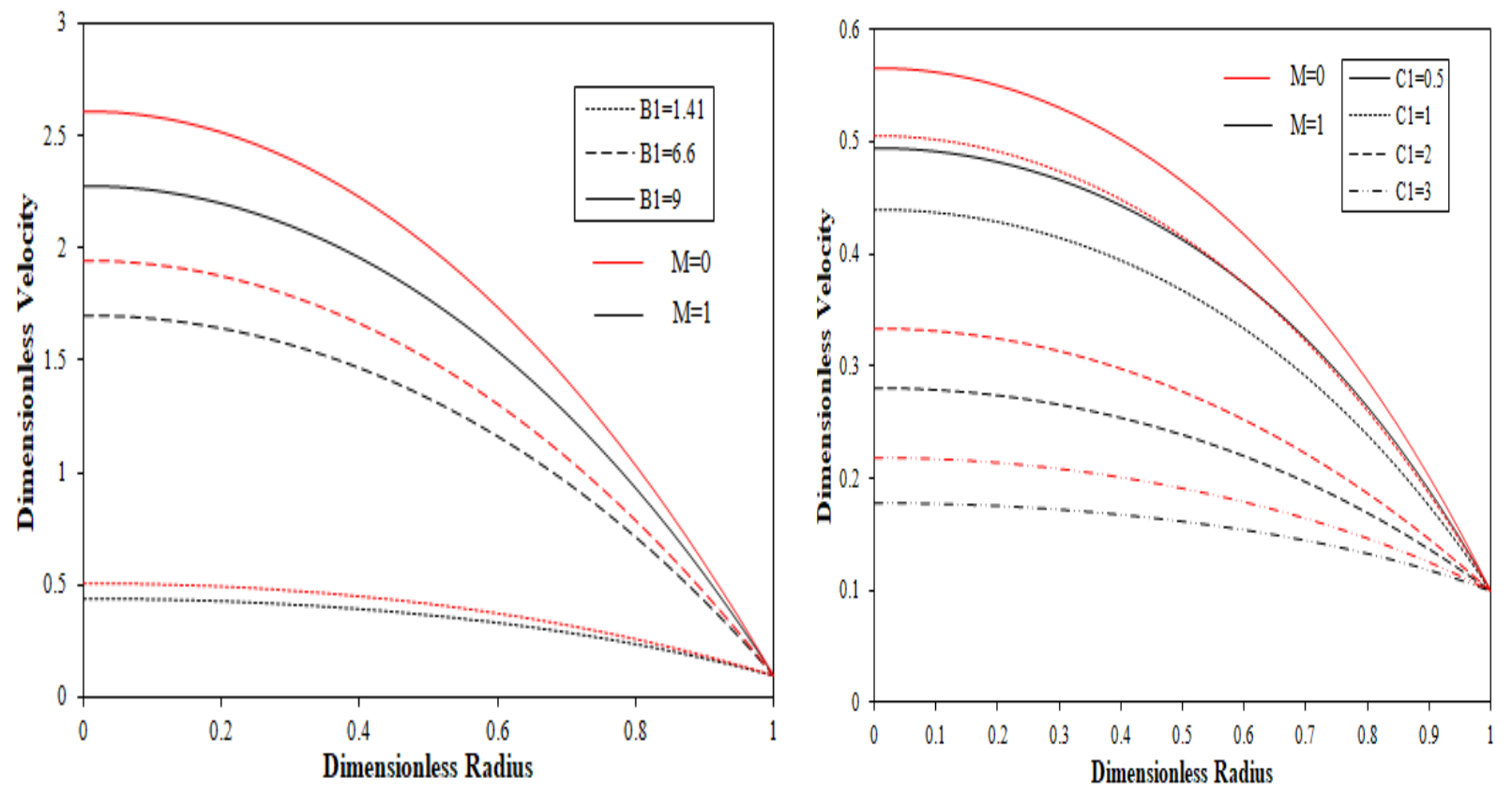

Figure 9: Effect on velocity profile for different (a) B1 parameter $(b) c_{1}$ parameter for following data $\delta=0.1, z=0.79, t=1.2$ 

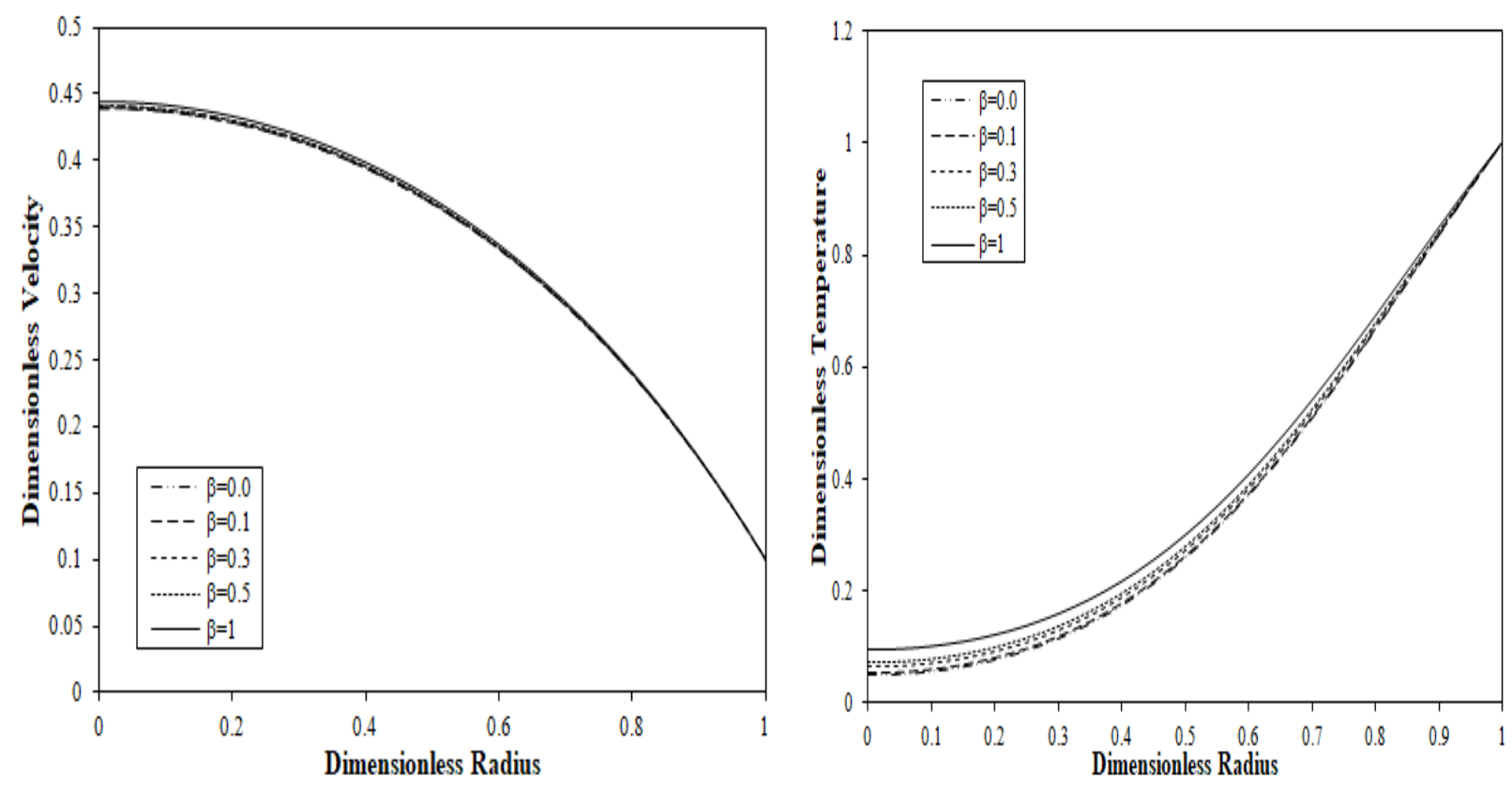

Figure 10: Effect of heat generation parameter $(\beta)$ on $(a)$ velocity and $(b)$ temperature profile for $B_{1}=1.41, \delta=$ $0.1, z=0.79, t=1.2$.

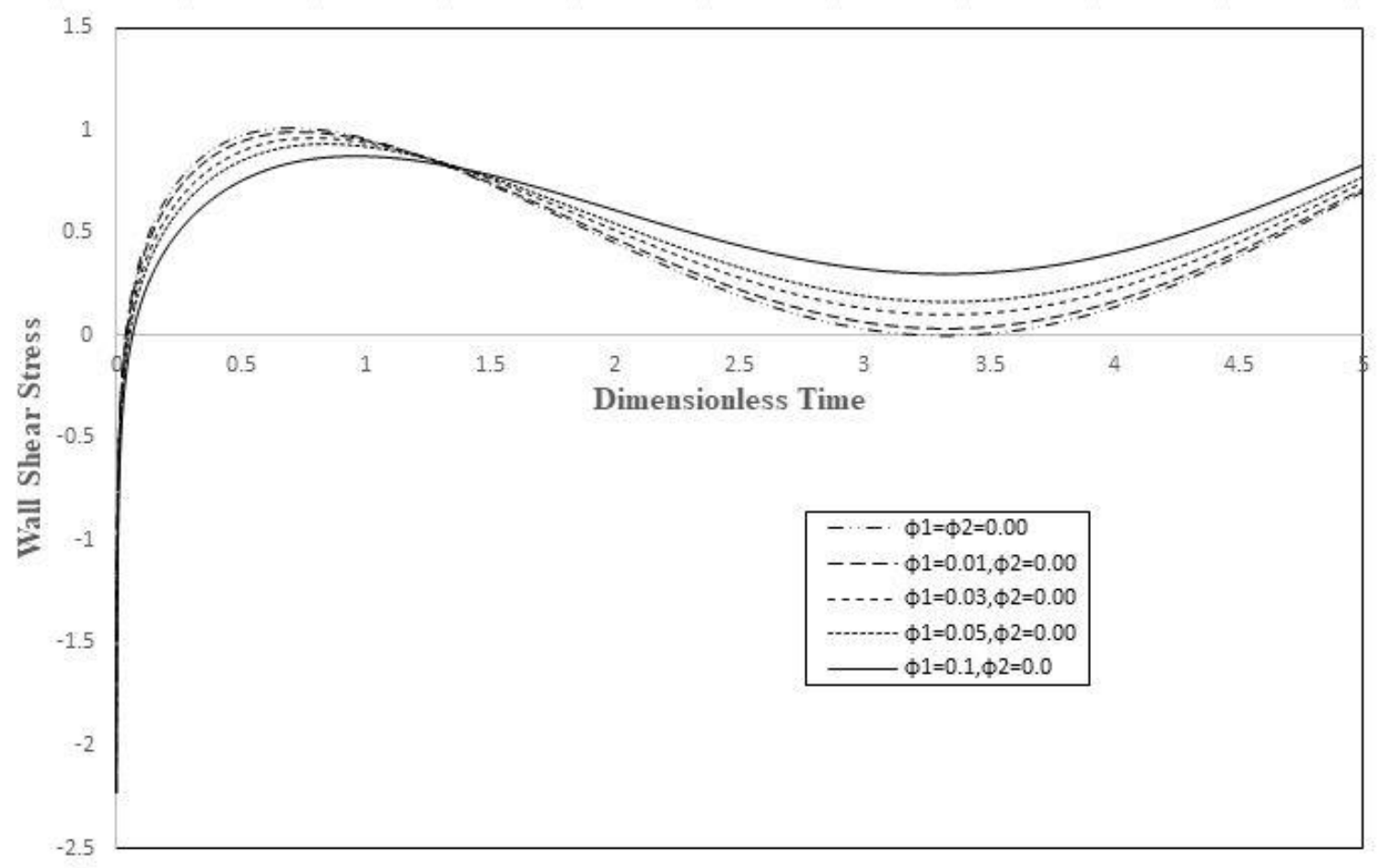

Figure 11: Wall shear stress for various nanoparticle concentrations of $A g$ nanofluid for $B_{1}=1.41, \delta=0.1, z=$ 0.79 . 


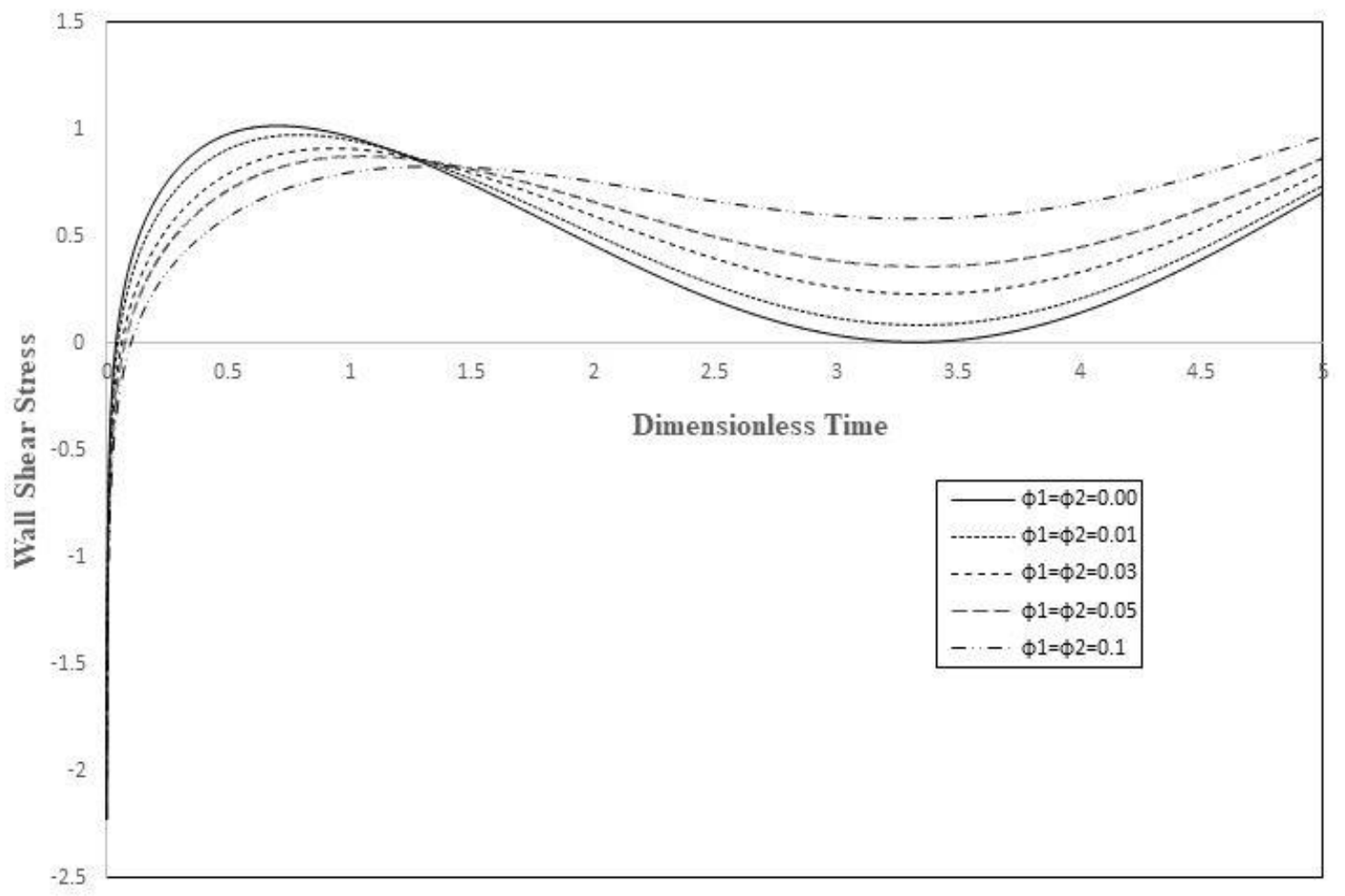

Figure 12: Wall shear stress (WSS) for various hybrid nanoparticle concentrations of $(A g+A u)$ nanofluid for $B_{1}=$ $1.41, \delta=0.1, z=0.79$.

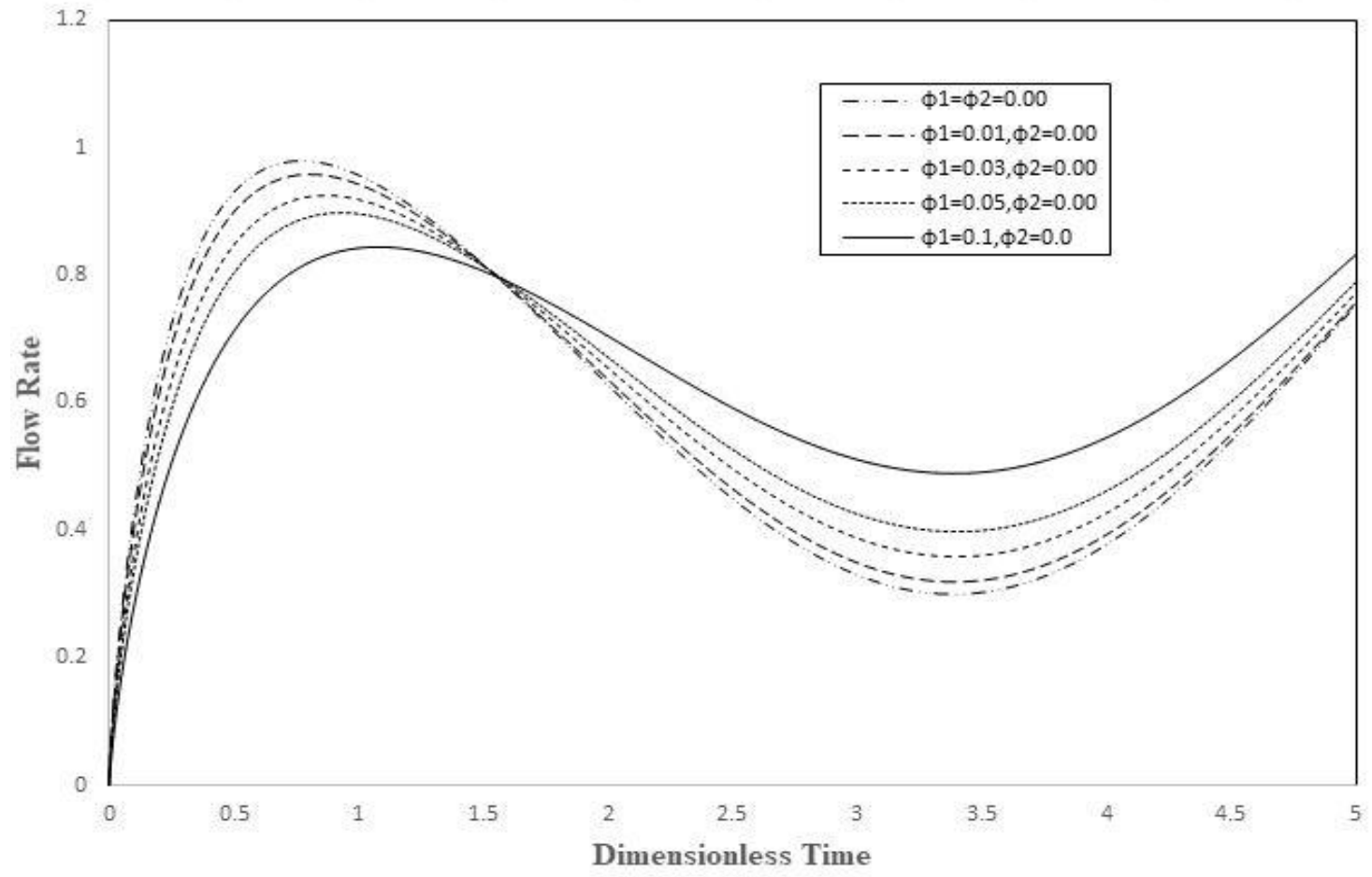

Figure 13: Flow rate for various nanoparticle concentrations of $A g$ nanofluid for $B_{1}=1.41, \delta=0.1, z=0.79$. 


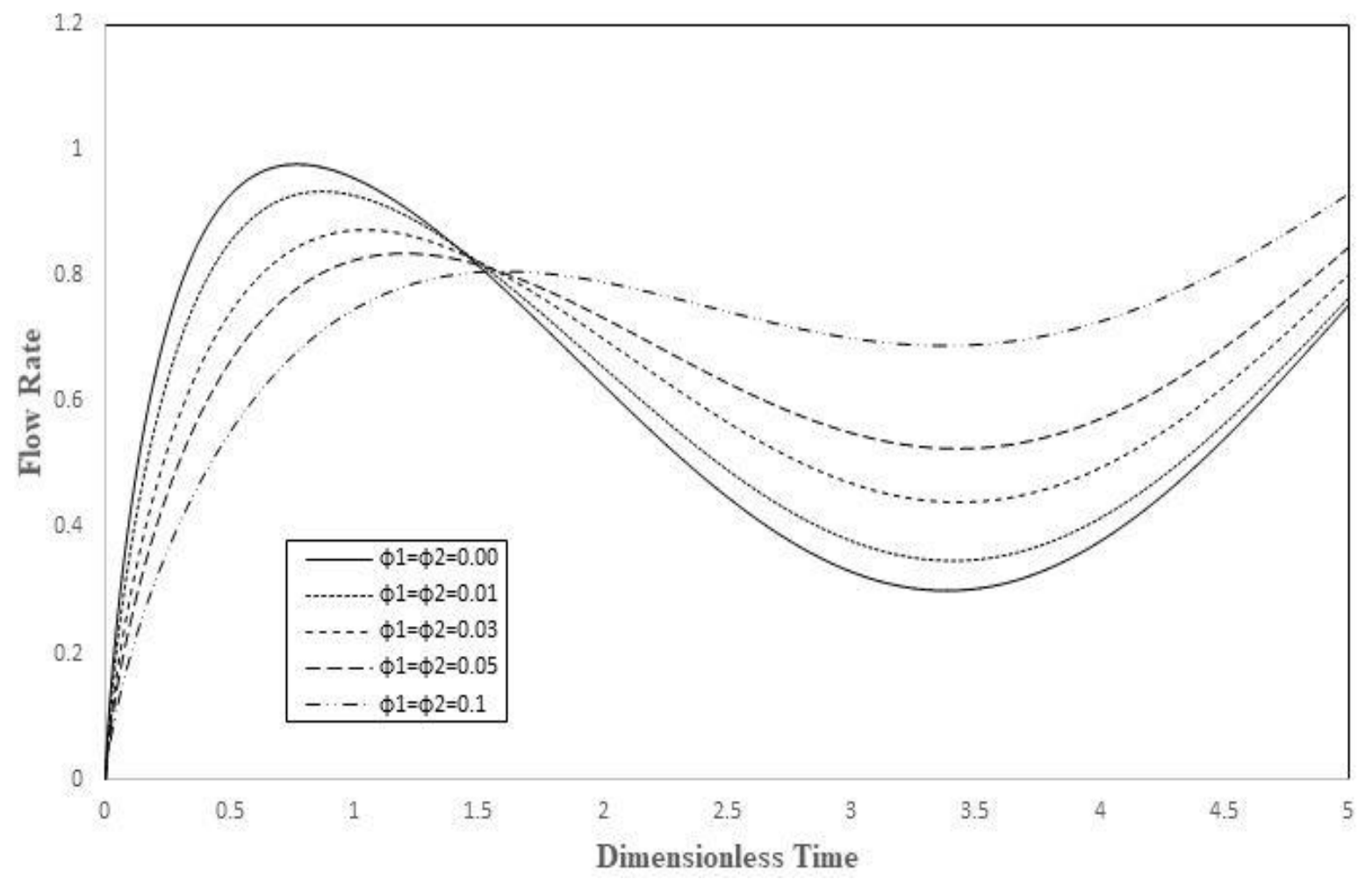

Figure 14: Flow rate for various hybrid nanoparticle concentrations of $(A g+A u)$ nanofluid for following data $B_{1}=$ $1.41, \delta=0.1, z=0.79$.

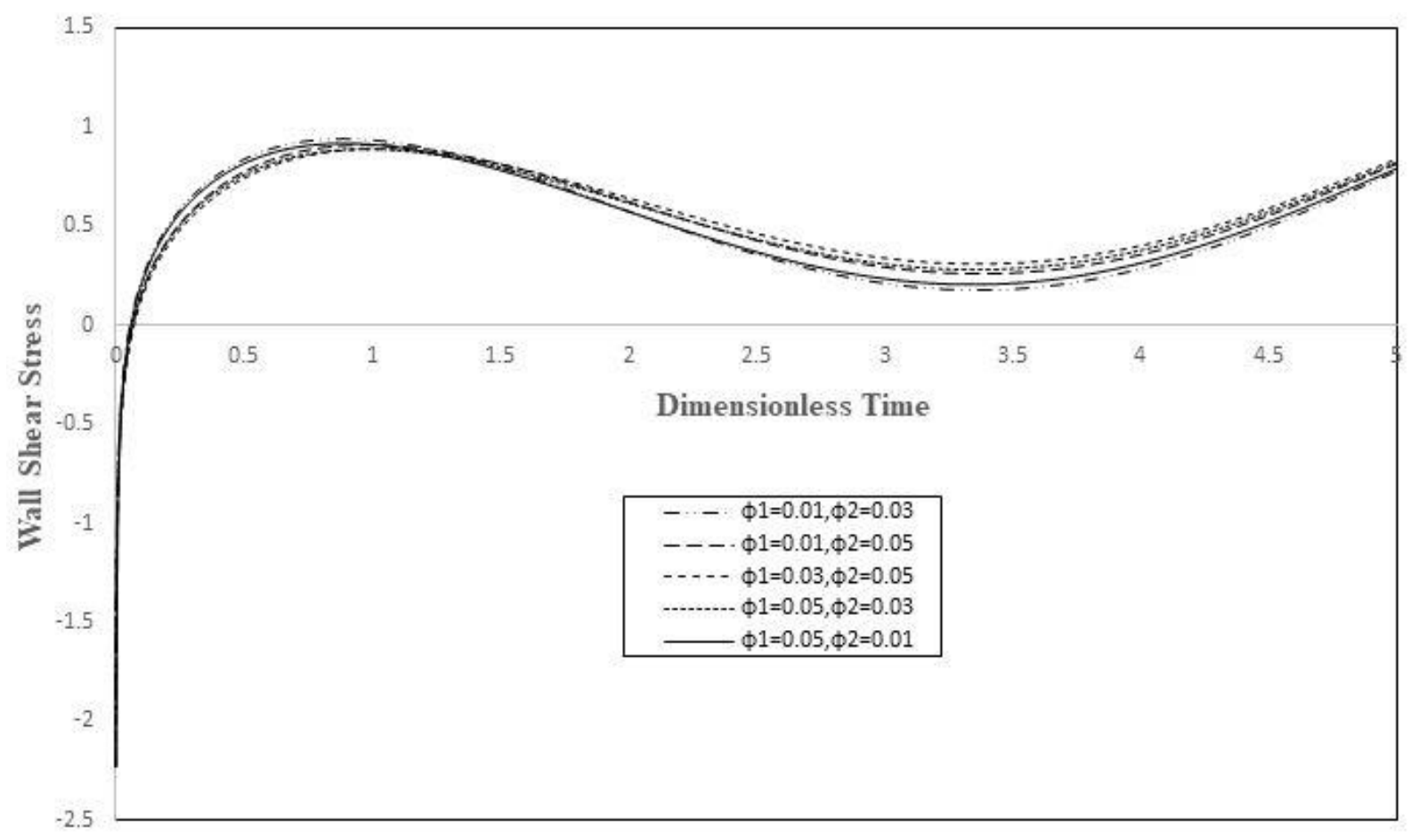

Figure 15: Wall shear stress of different concentration combinations of hybrid nanoparticles for

$$
B_{1}=1.41, \delta=0.1, z=0.79 \text {. }
$$




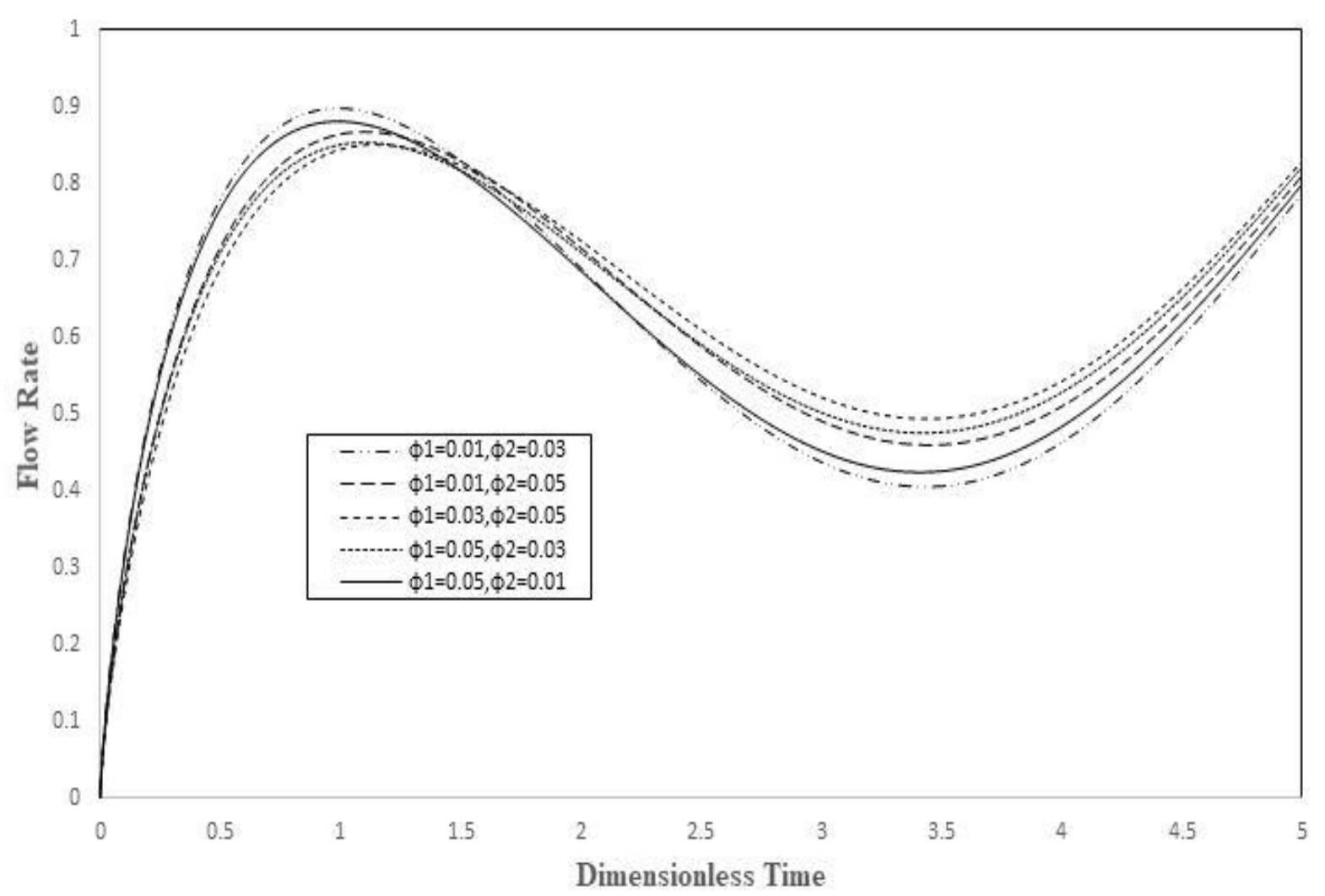

Figure 16: Flow rate of different concentration combinations of hybrid nanoparticles for $B_{1}=1.41, \delta=0.1, z=$ 0.79 .

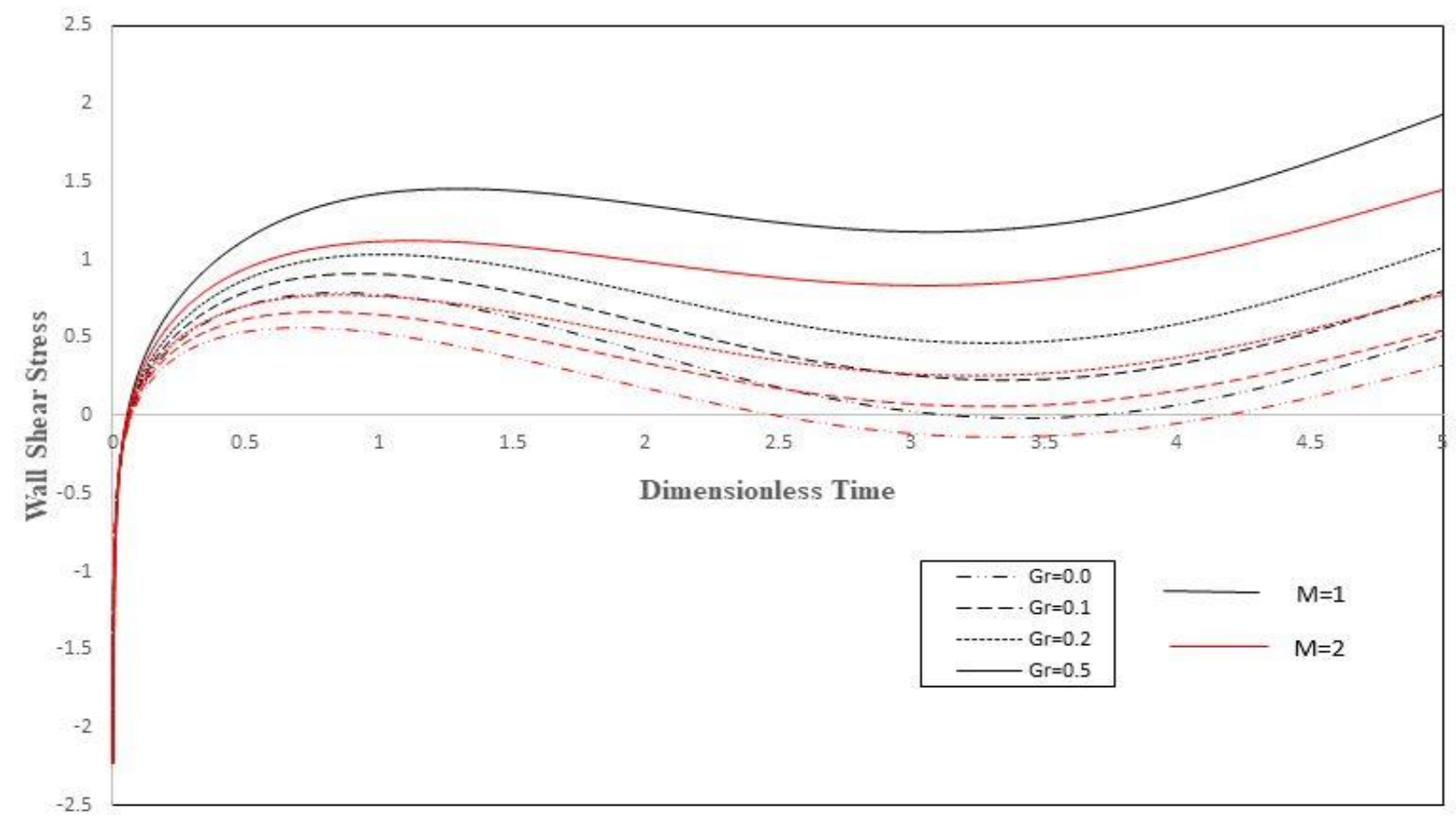

Figure 17: Wall shear stress for various Grashof numbers with $B_{1}=1.41, \delta=0.1, z=0.79$. 


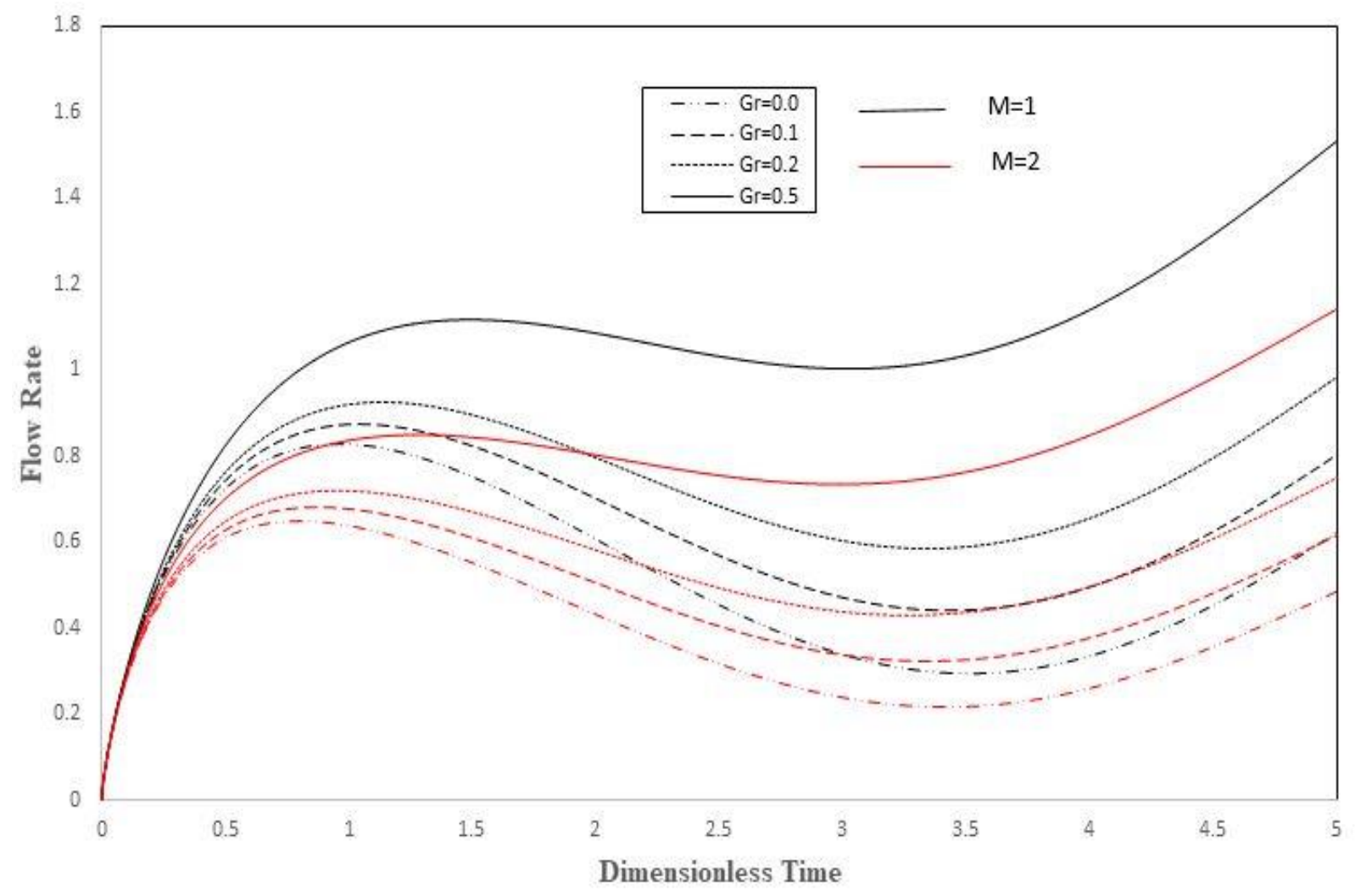

Figure 18: Flow rate for various Grashof numbers with $B_{1}=1.41, \delta=0.1, z=0.79$.

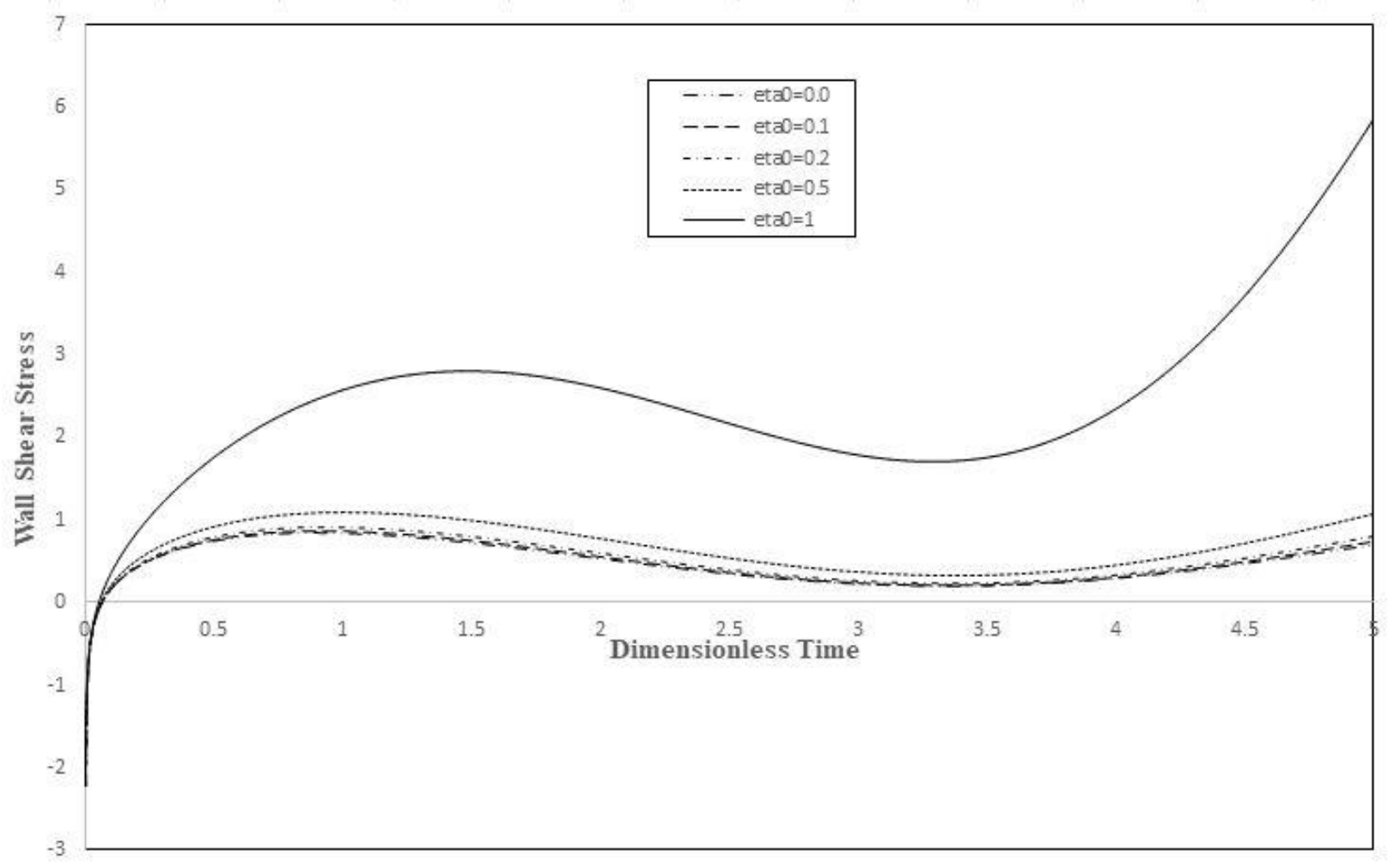

Figure 19: Wall shear stress (WSS) for various hybrid nanofluid viscosity parameter values with $B_{1}=1.41, \delta=$ $0.1, z=0.79$. 


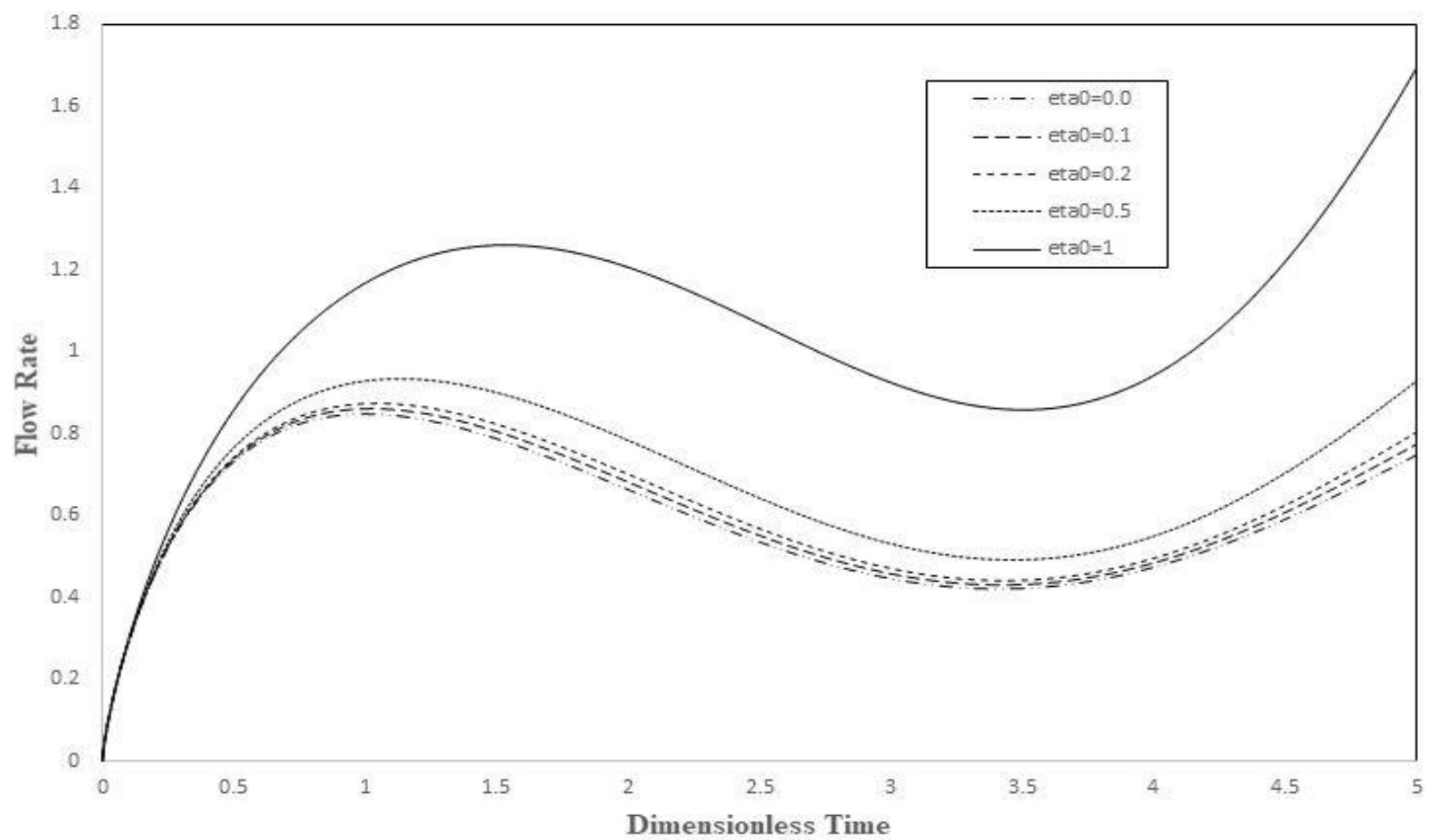

Figure 20: Flow rate for various hybrid nanofluid viscosity parameters with $B_{1}=1.41, \delta=0.1, z=0.79$.

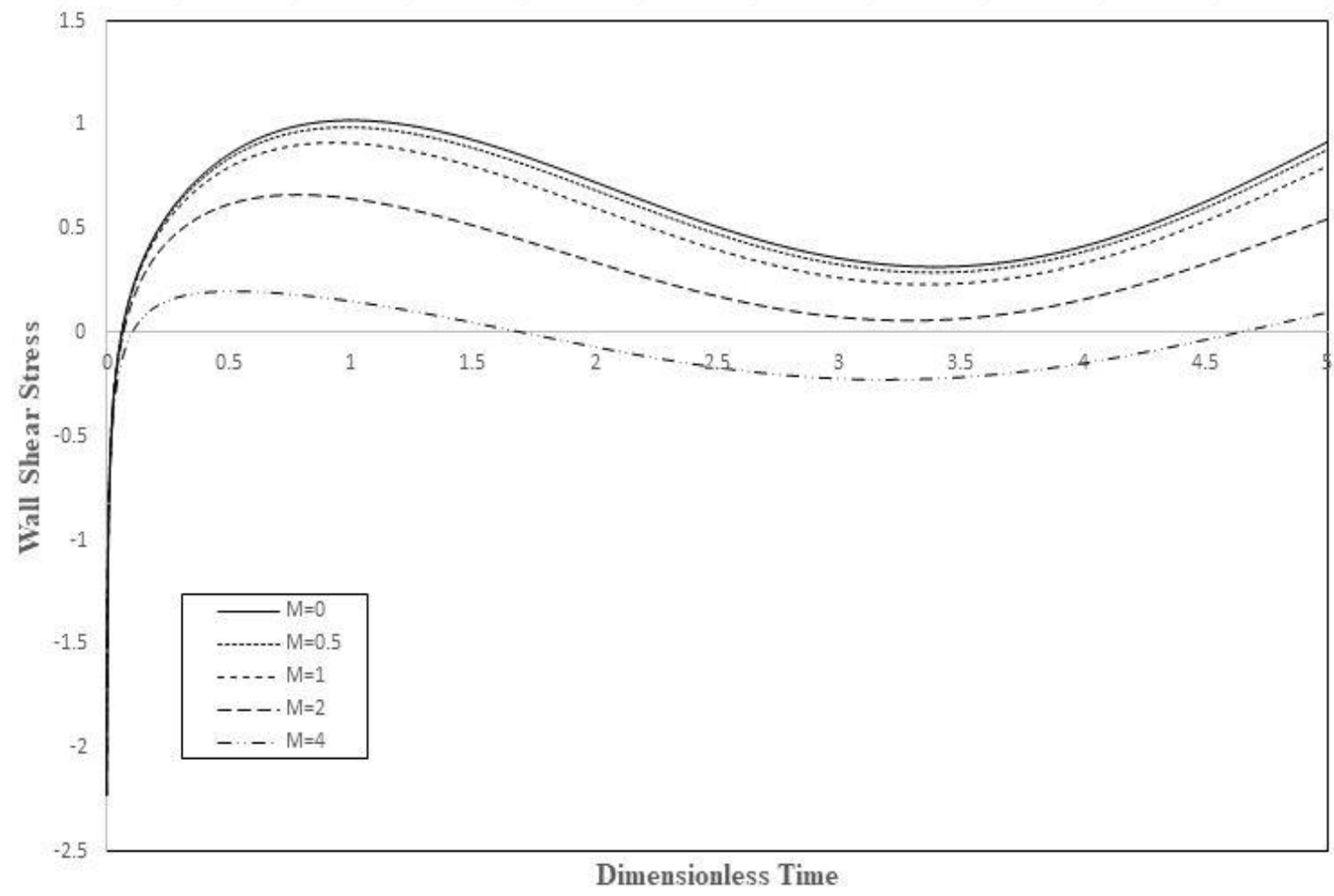

Figure 21: Wall shear stress for various magnetic parameters with $B_{1}=1.41, \delta=0.1, z=0.79$. 


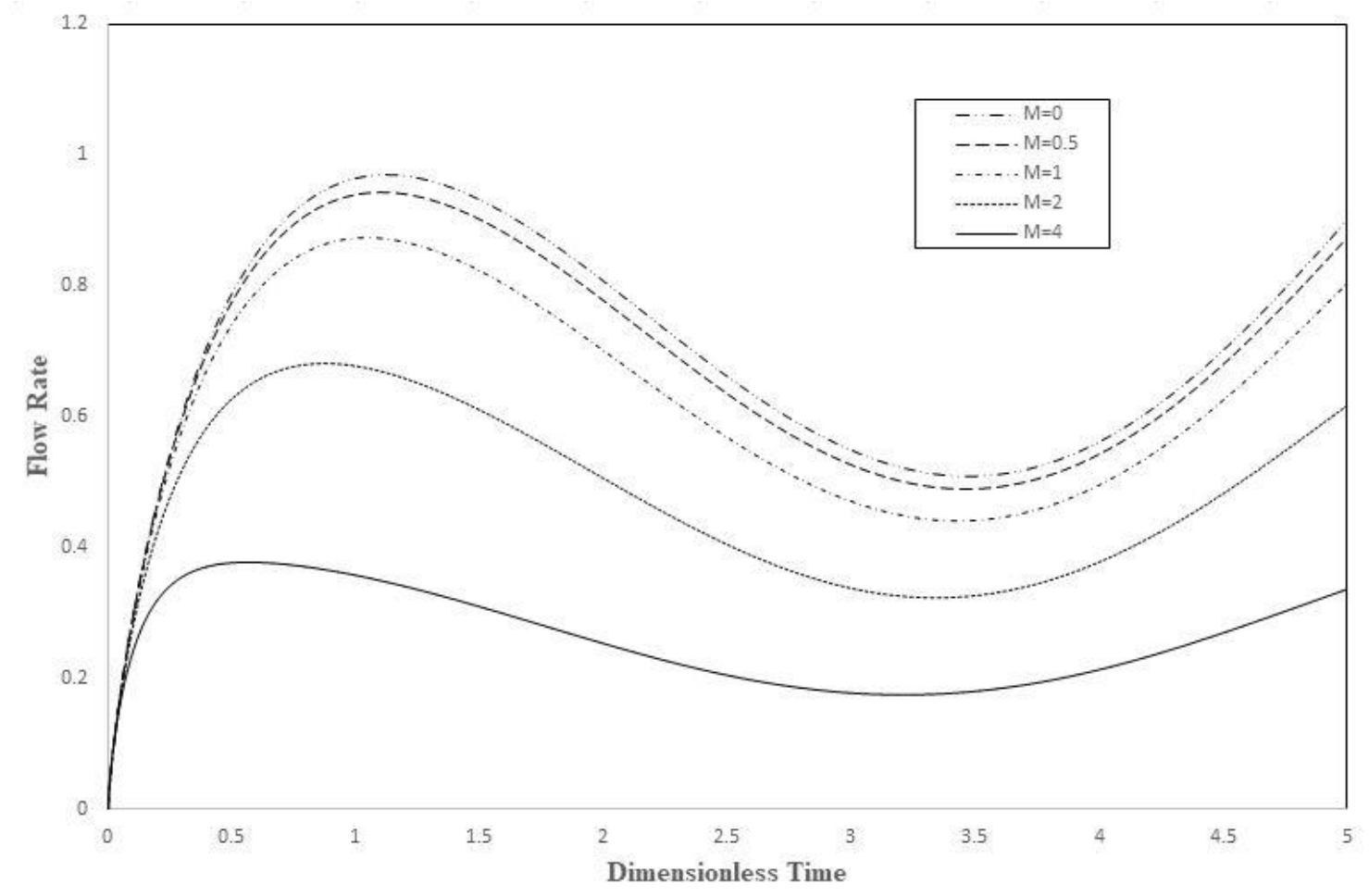

Figure 22: Flow rate for various magnetic parameters with $B_{1}=1.41, \delta=0.1, z=0.79$.

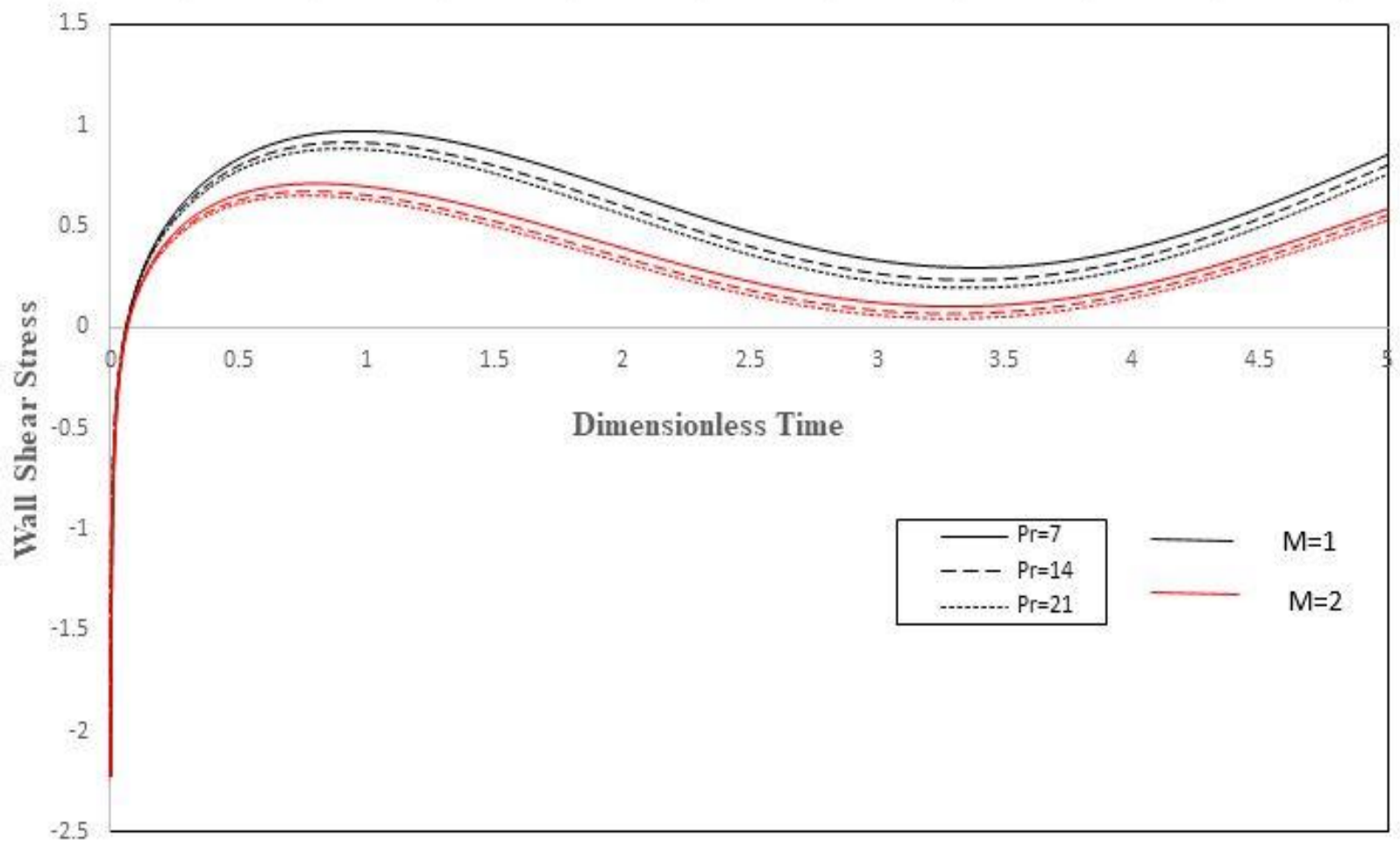

Figure 23: Wall shear stress for various Prandtl numbers with $B_{1}=1.41, \delta=0.1, z=0.79$ 


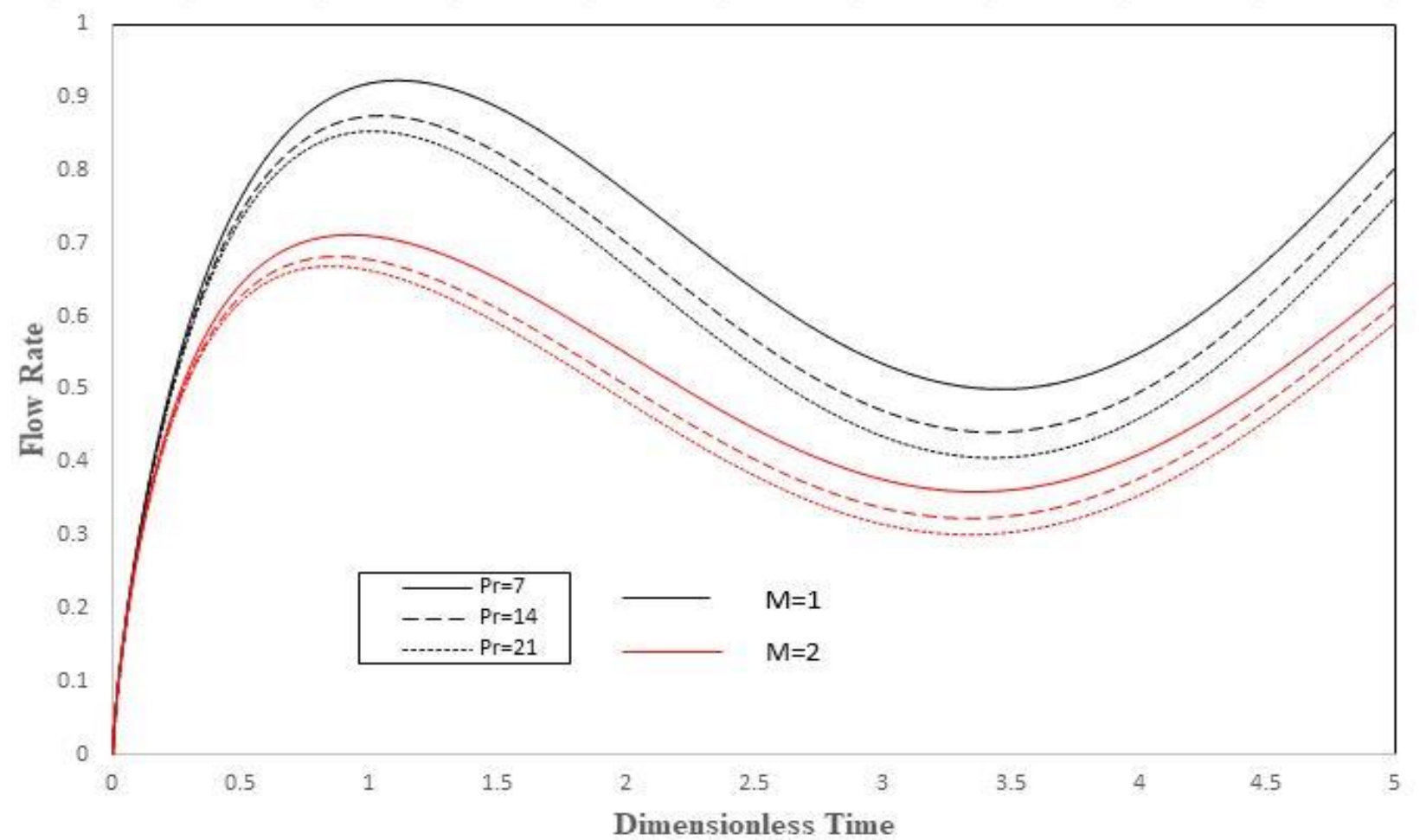

Figure 24: Flow rate for various Prandtl numbers with $B_{1}=1.41, \delta=0.1, z=0.79$.

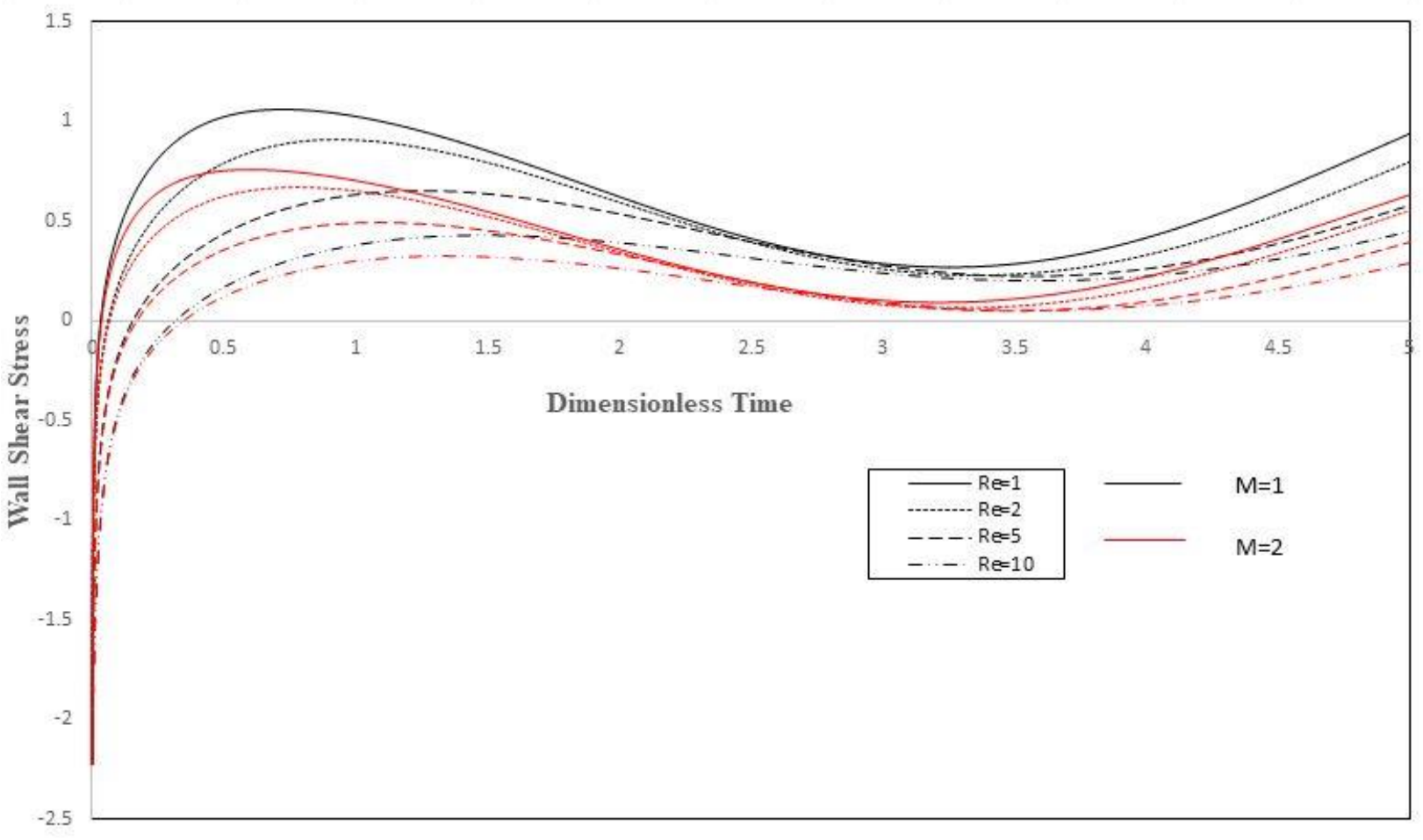

Figure 25: Wall shear stress for various Reynolds numbers with $B_{1}=1.41, \delta=0.1, z=0.79$. 


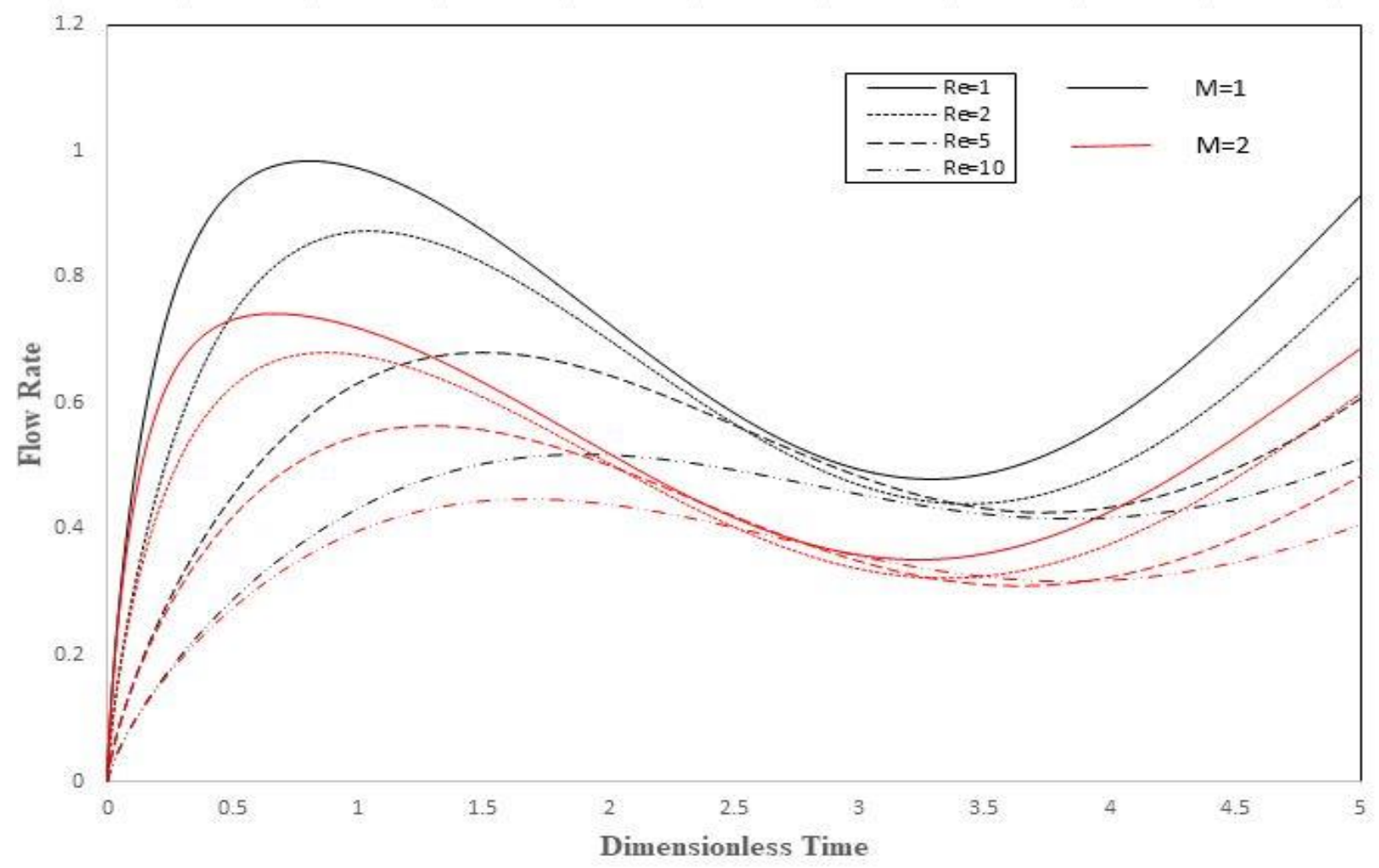

Figure 26: Flow rate for various Reynolds numbers with $B_{1}=1.41, \delta=0.1, z=0.79$.

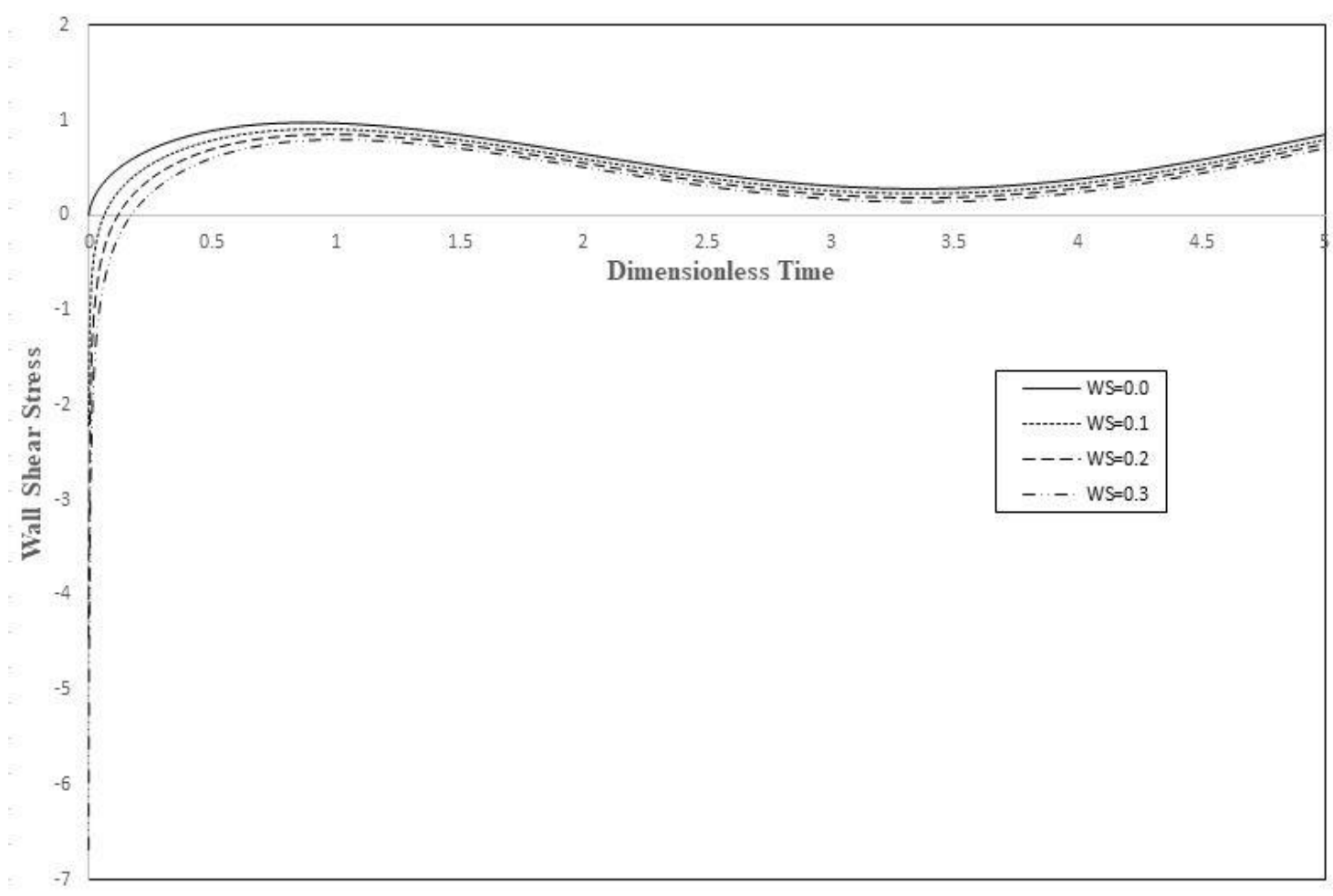

Figure 27: Wall shear stress for various slip velocity parameter with $B_{1}=1.41, \delta=0.1, z=0.79$. 


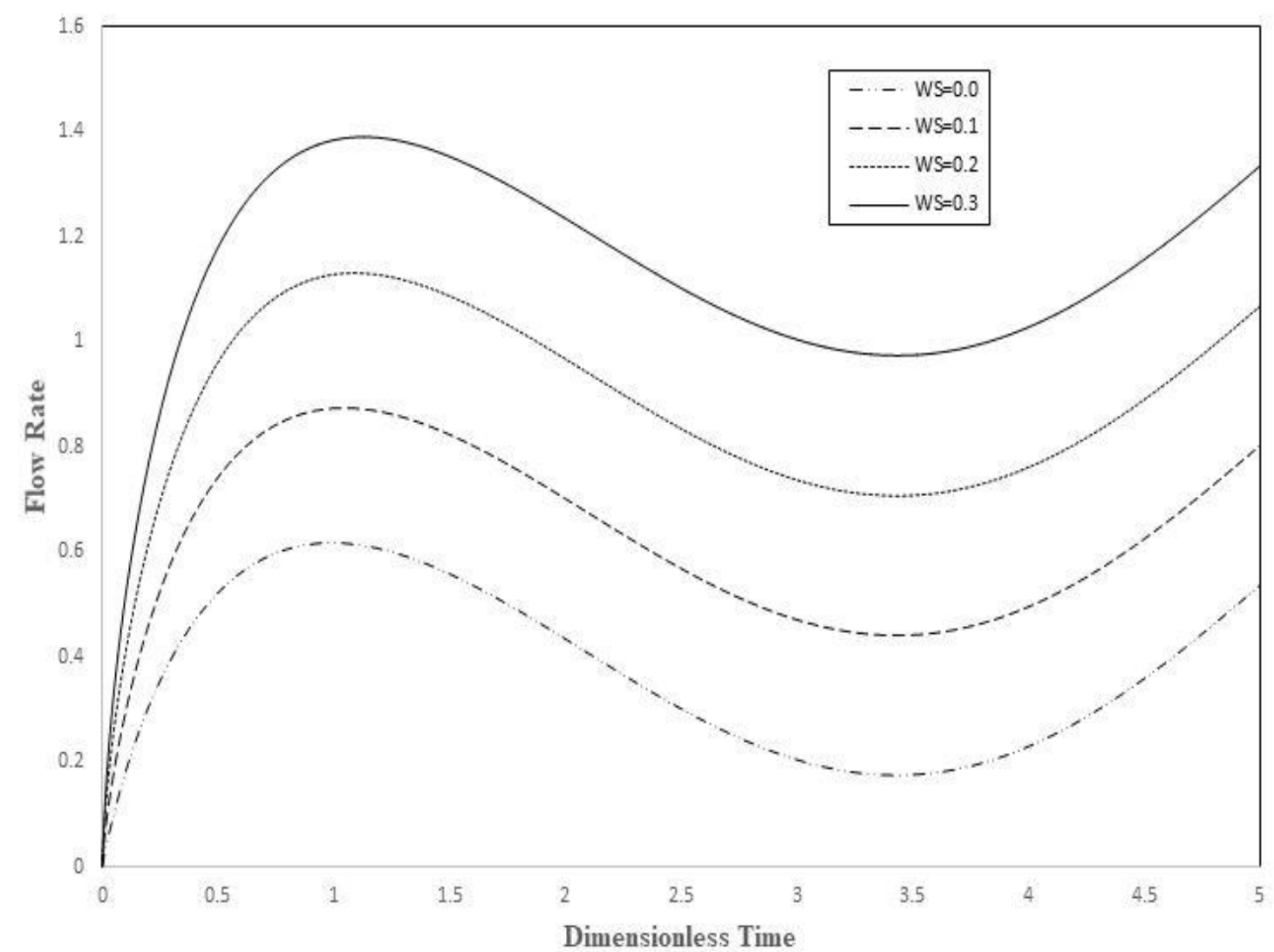

Figure 28: Flow rate for various slip velocity parameter with $B_{1}=1.41, \delta=0.1, z=0.79$.

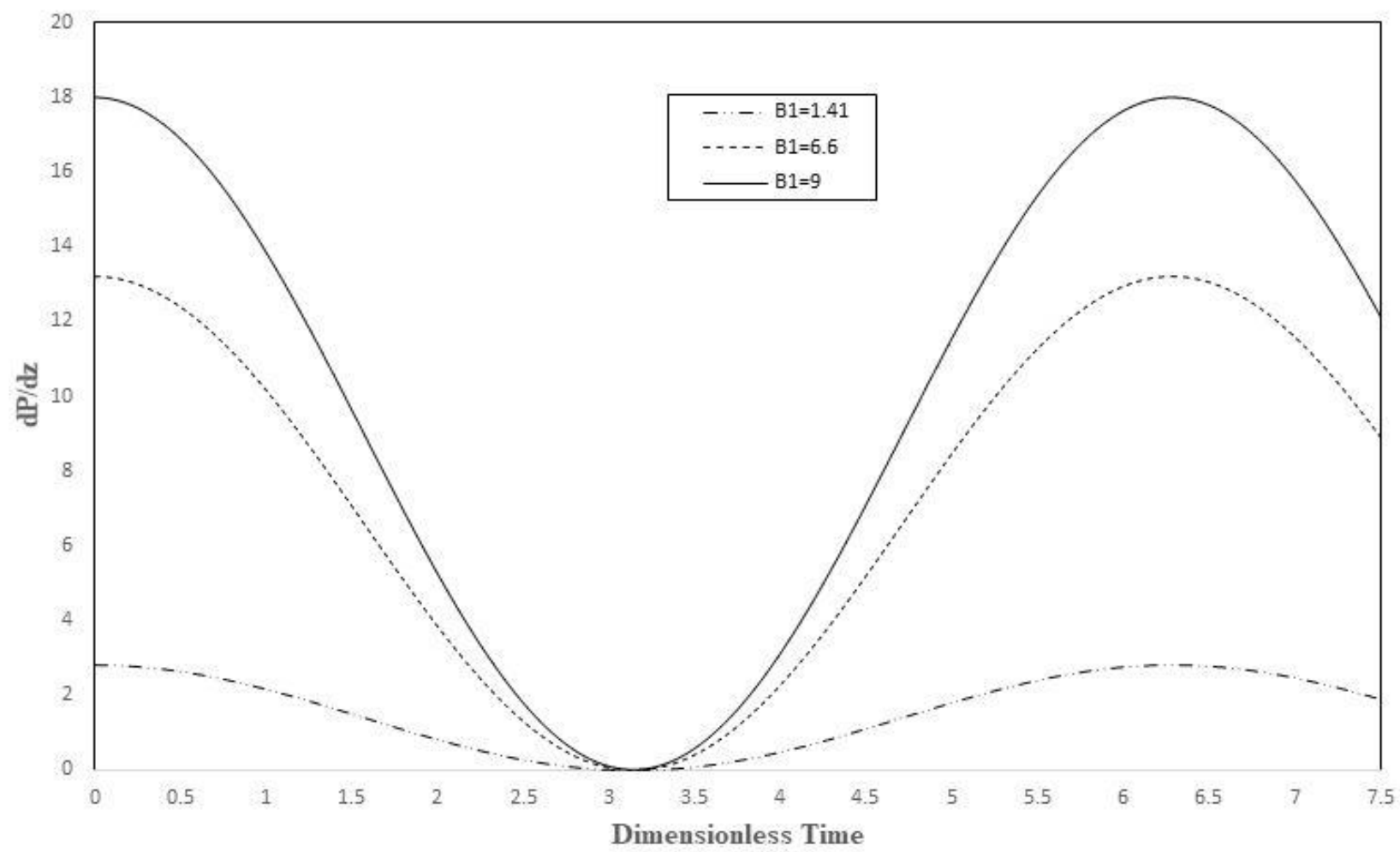

Figure 29: Pressure gradient for various $B_{1}$ parameters and $c_{1}=1, \delta=0.1, z=0.79$. 


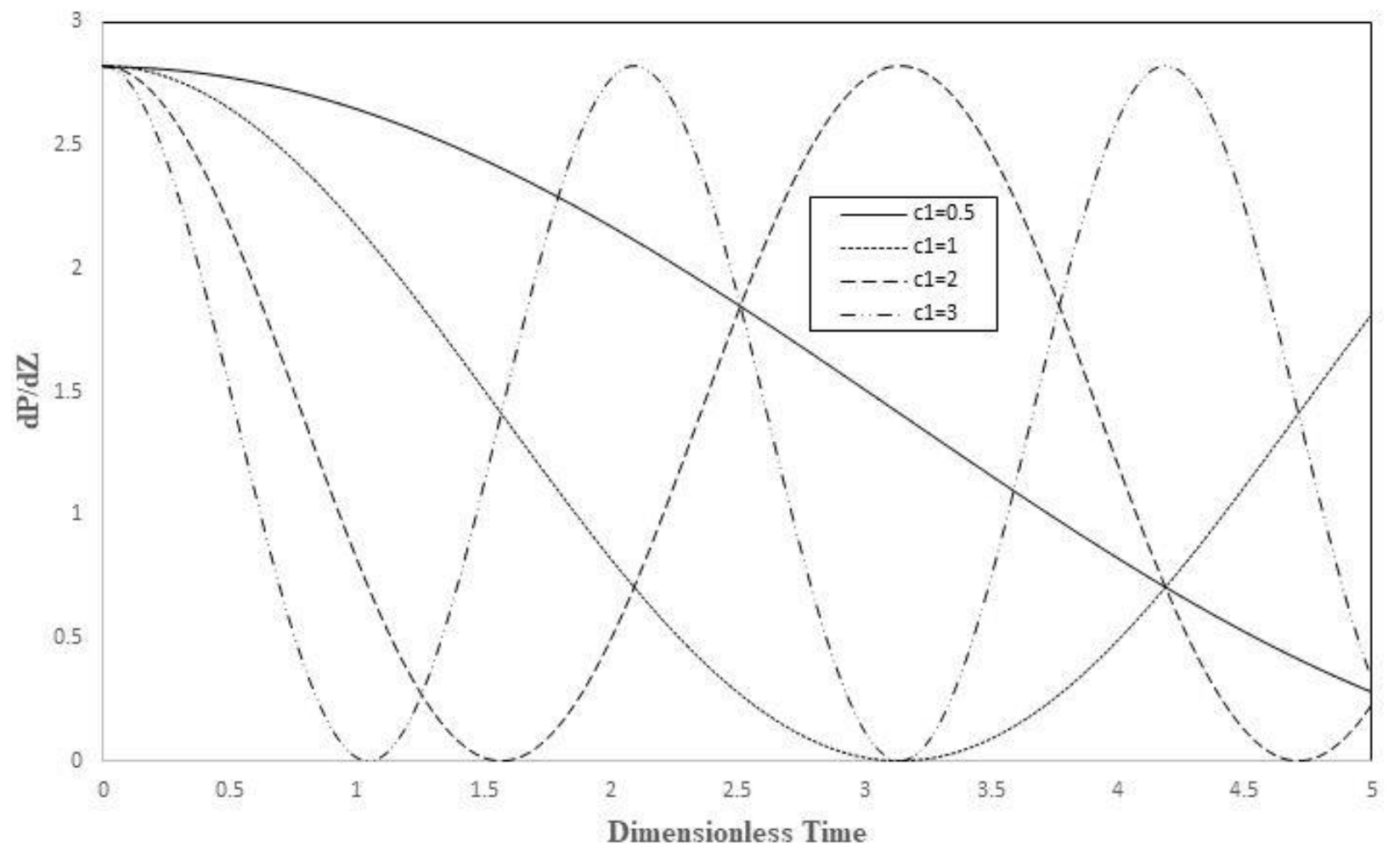

Figure 30: Pressure gradient for various $c_{l}$ parameters with $B_{1}=1.41, \delta=0.1, z=0.79$.

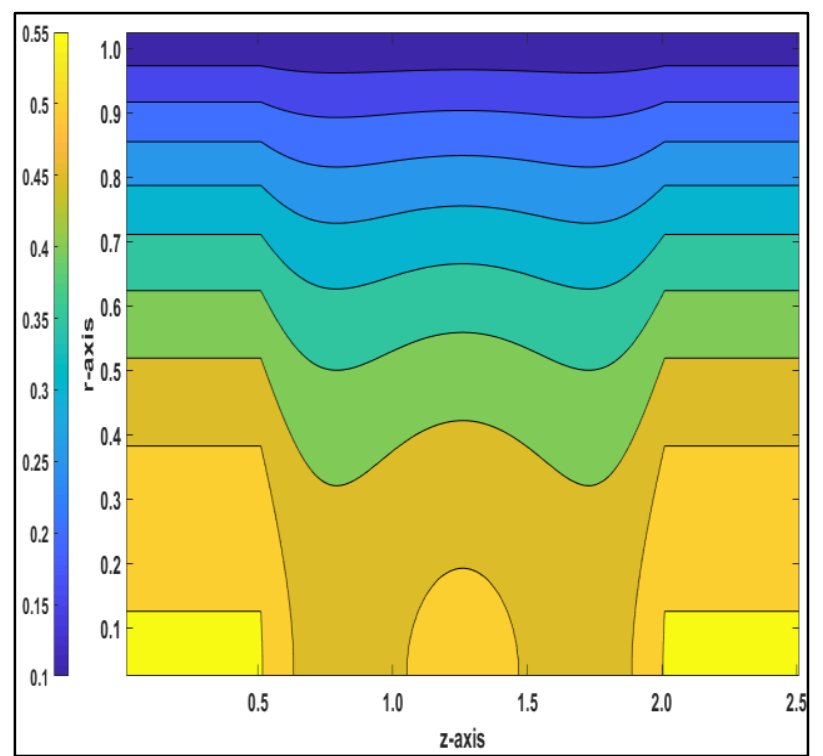

(a) For Blood $\phi_{1}=0.00, \phi_{2}=0.00$

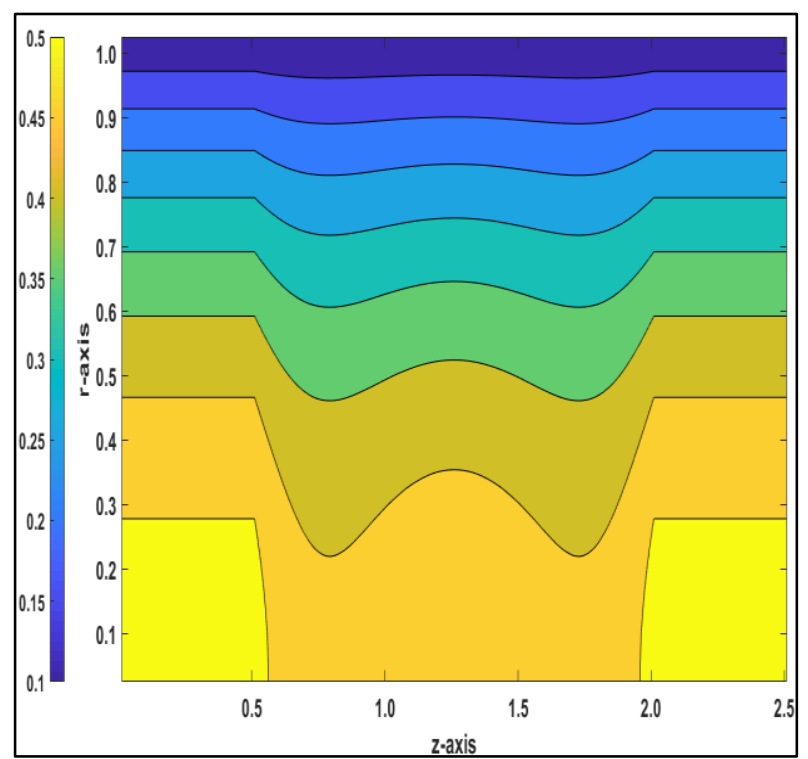

(b) For silver nano-fluid $\phi_{1}=0.03, \phi_{2}=0.00$ 


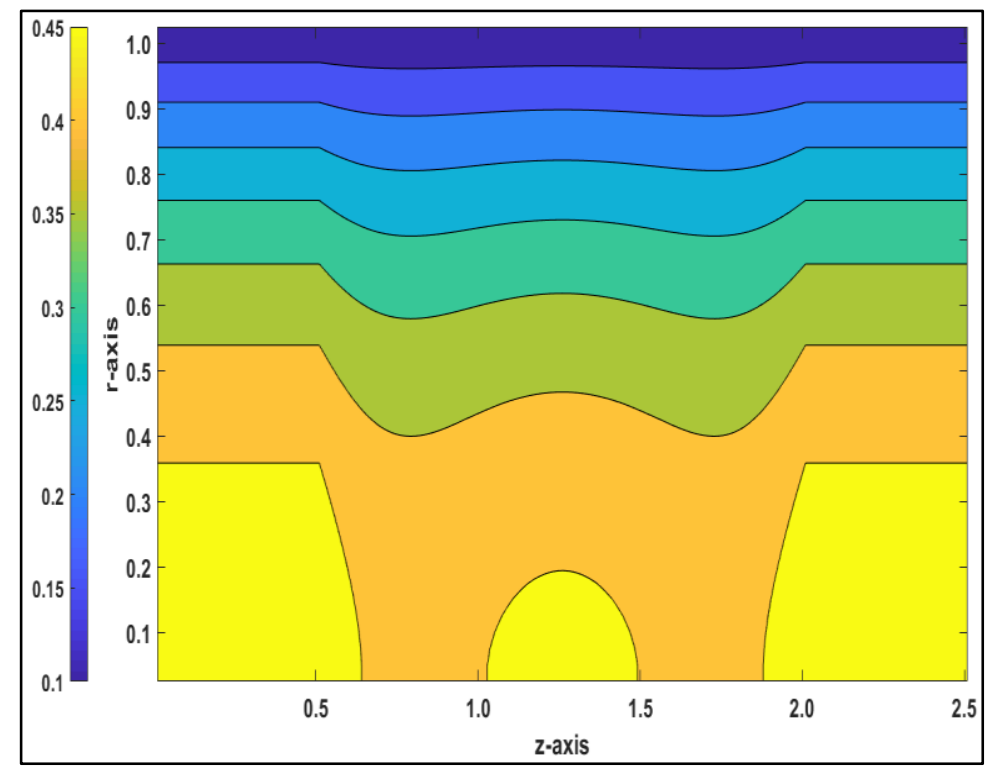

(c) For hybrid nano-fluid $\phi_{1}=0.03, \phi_{2}=0.03$

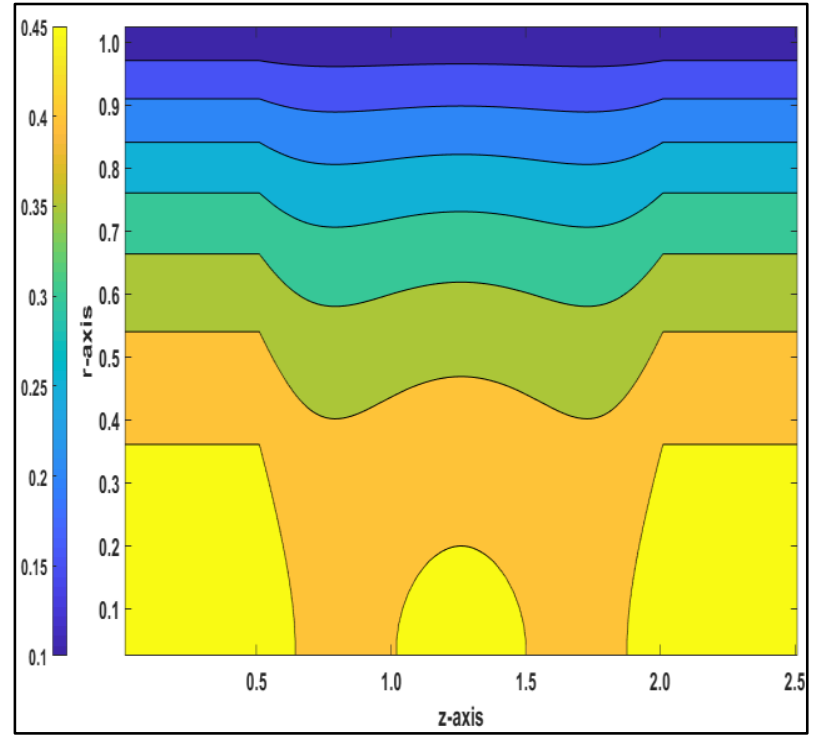

(d) On increasing $\beta, \beta=0.2$

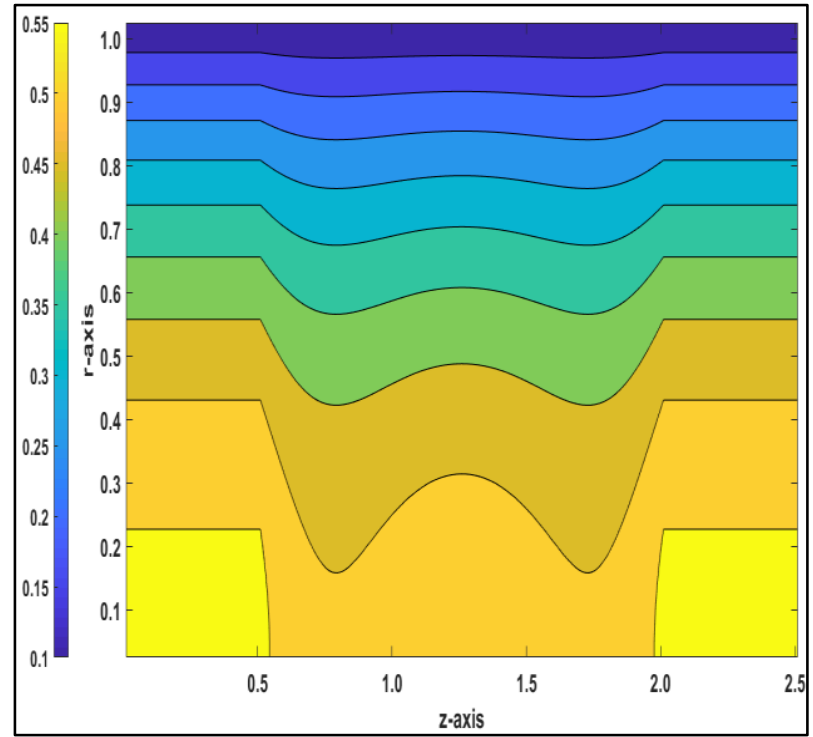

(e) Without magnetic field, $M=0$ 


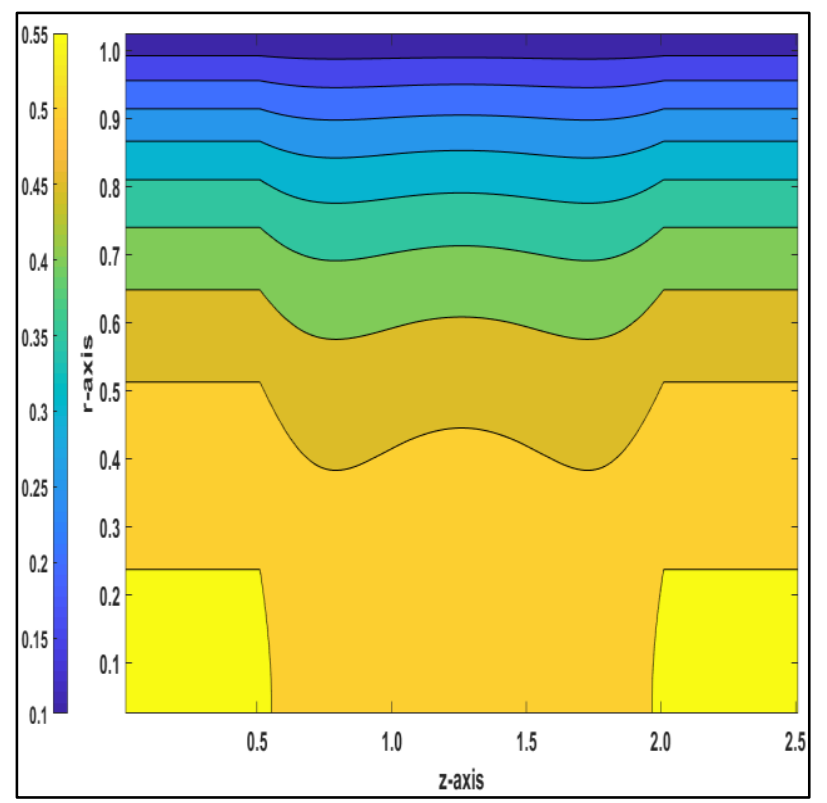

(f) On increasing $\mathrm{Gr}, \mathrm{Gr}=0.5$

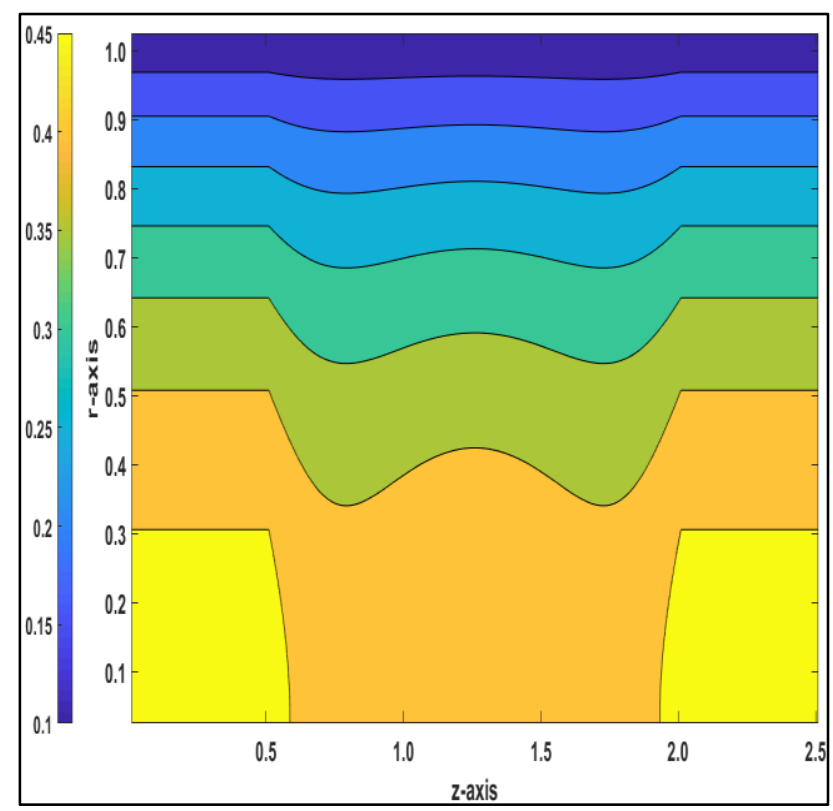

(h) On increasing $\mathrm{Pr}, \mathrm{Pr}=21$

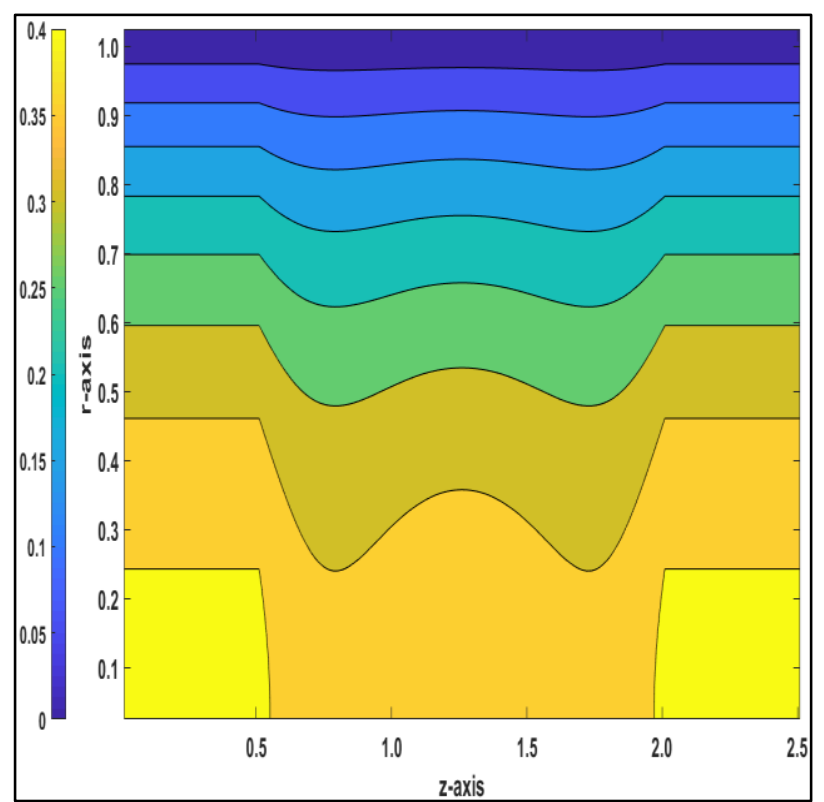

(g) For slip velocity $w_{S}=0.0$

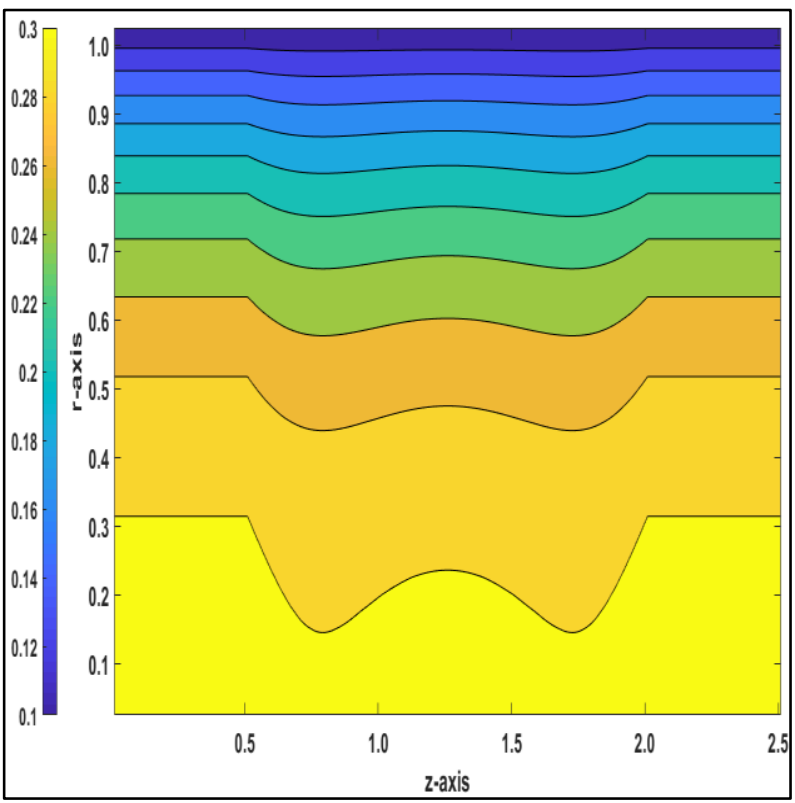

(i) On increasing $\mathrm{Re}, \mathrm{Re}=5$

Figure 31: Blood flow pattern along the axial and radial direction with $B_{1}=1.41, \delta=0.1, z=0.79$ 


\begin{tabular}{|c|c|c|c|}
\hline$M$ Variation & $\phi$ Variation & Silver nanofluid & Hybrid nanofluid \\
\hline $\mathbf{0 . 0}$ & 0.00 & 1.143032547 & 1.143032547 \\
\hline & 0.01 & 1.120093697 & 1.08779288 \\
\hline & 0.03 & 1.076754098 & 0.990900483 \\
\hline $\mathbf{1 . 0}$ & 0.05 & 1.035556804 & 0.916722806 \\
\hline & 0.00 & 1.013799016 & 1.013799016 \\
\hline & 0.01 & 0.995791633 & 0.972596841 \\
\hline & 0.03 & 0.960731104 & 0.89601051 \\
\hline & 0.05 & 0.927723281 & 0.834487738 \\
\hline
\end{tabular}

Table 4: Wall shear stress (WSS) for various emerging parameters at time $t=0.76$

\begin{tabular}{|c|c|c|c|}
\hline$M$ Variation & $\phi$ Variation & Silver nanofluid & Hybrid nanofluid \\
\hline $\mathbf{0 . 0}$ & 0.00 & 1.083293989 & 1.083293989 \\
\hline & 0.01 & 1.058809353 & 1.022325364 \\
\hline & 0.03 & 1.012325469 & 0.916791661 \\
\hline $\mathbf{1 . 0}$ & 0.05 & 0.969890666 & 0.837291548 \\
\hline & 0.00 & 0.977947071 & 0.977947071 \\
\hline & 0.01 & 0.958222542 & 0.930446509 \\
\hline & 0.03 & 0.920301065 & 0.844455101 \\
\hline & 0.05 & 0.885173741 & 0.77719006 \\
\hline
\end{tabular}

Table 5: Volumetric flow rate for various emerging parameters at time $t=0.76$ 

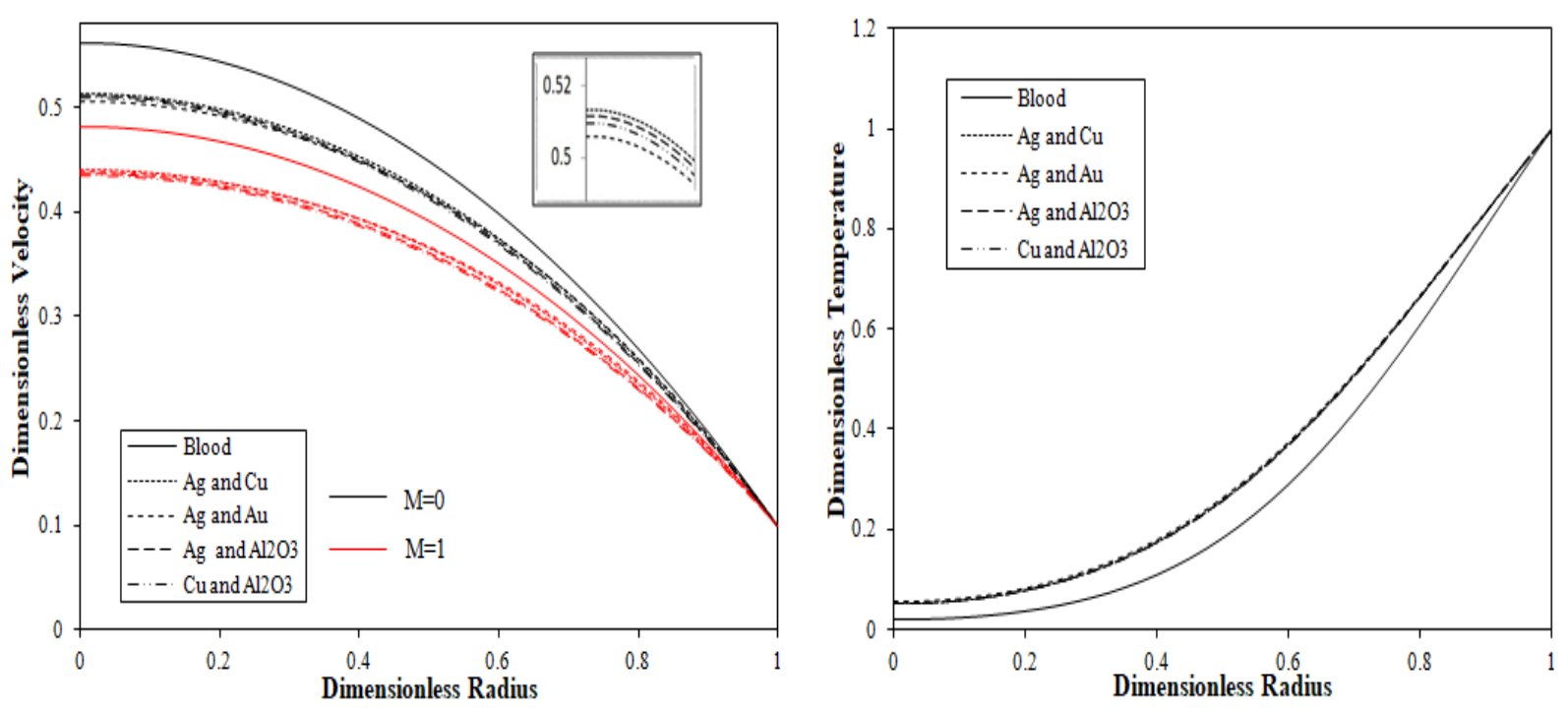

Figure 32: Effect of different types of hybrid nanoparticles on $(a)$ velocity and $(b)$ temperature profile for $B_{1}=$ $1.41, \delta=0.1, z=0.79, t=1.2$.

Figures 2 (a) - (b) displays the impact of various nanoparticle concentrations (volume fractions) on axial blood velocity component both with and without magnetic field. In figure 2(a), only silver nanoparticle is considered whereas fig 2 (b) corresponds to hybrid nanofluid. From both the figures, it is evident that an elevation in nanoparticle concentration produces marked flow deceleration. It is also evident that on applying stronger magnetic field in the radial direction the maximum velocity is also decreased. The presence of Lorentz magnetic drag force resists blood flow and generates retardation in the stenotic artery. In all plots, the velocity is maximum at the centerline of the artery and decreases to the vessel wall at which it attains the value of the slip velocity. On comparing both figures, it is apparent that addition of a second nanoparticle type exacerbates the decrement in velocity value and this result is extremely favorable for arterial diseases blood flow control.

Similarly, Figures 3 (a) - (b) is drawn for temperature profile considering different volume concentration of silver nanoparticles $\left(\phi_{1}\right)$ and hybrid (silver and gold) nanoparticles $\left(\phi_{1}, \phi_{2}\right)$ respectively. As volume fraction is increased, there is an apparent enhancement in temperature profile for both the cases at the throat of the stenotic artery. However, the higher increase can be observed for hybrid nanofluid in comparison with unitary nanofluid over an increment of nanoparticle concentration from 0.00 to 0.1 . It is also found that applied magnetic field shows no significant influence on temperature profile. Figure 4 illustrates the velocity and temperature 
profile for different combinations of hybrid nanoparticles $\left(\phi_{1}, \phi_{2}\right)$ both with $(M>0)$ and without $(M=0)$ magnetic field. It is evident that on changing the nanoparticle concentrations both $\phi_{1}$ and $\phi_{2}$ from 0.01 to 0.05 , a significant amount of reduction in velocity is induced. Among the various combination of nanoparticle concentrations, the highest decrement in axial velocity is computed for $\phi_{1}=0.03$ and $\phi_{2}=0.05$. Similarly, figure 4 (b), indicates that temperature magnitudes are boosted with increment in volume fraction of both nanoparticles. As with the velocity distributions, the nanoparticle concentrations $\phi_{1}=0.03$ and $\phi_{2}=0.1$ are associated with the greatest change in temperature value i.e. the highest thermal enhancement is achieved for this case which is attributable to the strong accentuation in thermal conductivity of the nanoparticle-doped blood.

Figures 5 (a) - (b) depict the evolution in velocity profiles with a variation in magnetic parameter and wall slip velocity, respectively. It is observed that increase in the value of magnetic parameter $(M)$ decreases the blood flow velocity around the centerline of the artery. Therefore, stronger magnetic field elevates the Lorentzian hydromagnetic drag which induces flow deceleration in the stenotic region. Effective flow control in biomedicine can therefore be achieved via externally imposed magnetic fields. In figure 5 (b), with increasing slip parameter, acceleration in axial velocity is noticed. The slip parameter is a boundary condition which is imposed at the inner surface of the arterial wall. The presence of the slip velocity induces a momentum boost which assists the blood in the near-wall regime and accelerates blood flow, although the effect clearly decays with further radial distance towards the arterial centre-line.

The velocity profiles for different nano-fluid viscosity parameter $\eta_{0}$ and Grashof number $G r$ are plotted in figure 6 (a)-(b). These figures show that a similar response is induced with increment in both parameters $\left(\eta_{0}, G r\right)$, i.e. the magnitude of the axial velocity is enhanced and strong flow acceleration is attained in the regime. In figure 6 (a), the range of nano-fluid viscosity is taken as 0.0-1.0 while for figure 6(b), Grashof number is taken in range of 0.0-0.5. The acceleration in velocity shows that the blood viscosity or resistance among the particles has reduced for the more magnitude of parameter $\left(\eta_{0}\right)$. The case $\eta_{0}=0$ implies constant viscosity i.e. no variation and $\eta_{0}=$ 1 is associated with exponential viscosity decrease. Velocity diminishes with lower values of viscosity parameter. This is attributable to the increase in viscous force relative to inertial forces 
with greater viscosity which decelerates the flow. Further, Grashof number $(G r)$ is the ratio of buoyancy force to viscous force in the hemodynamic regime. Higher thermal buoyancy assists momentum diffusion and accelerates the blood flow. Lower resistance is therefore offered inside the blood and strong enhancement in velocity is computed. Evidently flow deceleration arises with vanishing thermal buoyancy force $(G r=0)$ which corresponds to forced convection, as seen in Fig. 6b.

Figure 7 (a)-(b) shows the profile of velocity and temperature for various Prandtl numbers $(P r)$ and for two different magnetic field parameter values. Both figures demonstrate that flow deceleration and cooling is induced with increasing Pr. Prandtl number is the ratio of momentum diffusivity to thermal diffusivity, which implies that $\mathrm{Pr}$ is inversely related to heat transfer from the artery wall to the fluid. When $\operatorname{Pr}$ is significantly small $(<1)$, the diffusion of heat is greater than momentum. The curve trend in Figure 7 (a)-(b) confirms that with higher Prandtl number, velocity plummets as does temperature magnitude (this will correspond to thicker momentum boundary layer at the arterial wall and a thinner thermal boundary layer). It is noteworthy that $\operatorname{Pr}=21$ approximately falls within the correct range for laminar streaming blood at room temperature, i.e. 19.6-30.8 as noted by Mitvalský [43]. Blood therefore has a much higher momentum diffusivity compared with thermal diffusivity, which is necessary for homeostasis (thermoregulation) and other biothermal functions as elaborated by Diller [44] and Hensley et al. [45]. It is also seen that on increasing the magnetic parameter, velocity decreases significantly whereas there is no tangible impact on temperature profile.

Figures 8 (a)-(b) visualize the impact of Reynolds number $(R e)$ and magnetic parameter $(M)$ on blood flow velocity and temperature profile. It is noteworthy that very low Reynolds numbers are considered (laminar flow) and the regime is therefore a viscous dominated one. The computations show that on increasing the value of $\operatorname{Re}$ from 1 to 10, a significant amount of reduction in velocity has been seen. In the same manner, as we increased the magnitude of $R e$, the magnitude of temperature is also decreased. Although inertial force is increased with Reynolds number (based on the vessel radius), the overwhelming effect is nevertheless flow deceleration owing to the stenotic obstruction and nanoparticles, which manifests in significant impedance to the streaming blood. Again, stronger magnetic field $(M)$ also induces flow retardation. Temperatures are also suppressed with greater Reynolds number and the profiles become increasingly of a lesser 
gradient, becoming attached the zero line with subsequent temperature growth from the wall taking increasingly longer radial distances.

Figure 9 (a)-(b) portray the influence of pressure gradient (pulsatile flow) parameters $B_{1}$ and $c_{1}$ (featured in the non-dimensionalized form of the pressure gradient equation) on velocity and temperature distributions. For different sizes of artery, such as arterioles or the coronary artery, the value of $B_{1}$ is 1.41 ; however for the femoral artery of the human body, $B_{1}$ attains a much higher value of 6.6, at a particular location 'throat' of the stenotic artery (see Burton [41]). Figure 9 (a) implies that on increasing the value of $B_{1}$ parameter, the blood flow is significantly accelerated confirming that in large (femoral) arteries the velocity is higher relative to smaller (coronary) arteries. Effect of $c_{1}$ parameter on the velocity profile is illustrated in Figure 9 (b). It is noticed that at time $t=1.2$, velocity decreases significantly with elevation in the value of $c_{1}$ from 0.5 to 3. Therefore, marked flow retardation is induced with the $c_{1}$ pulsatile parameter.

The effect of heat generation parameter $(\beta)$ on velocity and temperature profile is presented in figure 10 (a) - (b). This effect may correspond for example to spot thermal therapy in laser treatment $[46,47]$. An increase in this parameter shows a comparatively minimal impact on velocity profile (although a weak acceleration is mobilized) whereas it produces a much more prominent enhancement in temperature, in particular, near the centerline of the arterial zone. Towards the vessel periphery (large radial coordinate) there is a progressive diminishing in the influence of heat generation and all profiles merge.

Figure 11- 12 are plotted to display the time series of wall shear stress for variation in both nanoparticle concentrations. These figures capture the oscillatory nature of the blood flow in the stenotic region and allow a direct comparison between the performance of silver nanofluid $\left(\phi_{1}\right)$ and hybrid nanofluid $\left(\phi_{1}+\phi_{2}\right)$ with progression in time. Both figures show that wall shear stress initially decreases and after a critical point in time, assumes an ascending trend which is sustained for all subsequent time, with increasing volume concentrations for any of the nanoparticles. Lower WSS magnitudes are computed for the first part of the cycle at nanoparticle concentration of 0.1 . Nevertheless, comparing both the figures, it is evident that for the case of hybrid nanoparticles $\phi_{1}=0.1, \phi_{2}=0.1$, wall shear stress is lower in comparison to the uni-nanoparticle case, $\phi_{1}=0.1$. Figures (13)-(14) exhibit the impact of various nanoparticle concentrations on the volumetric flow rate. This quantity also significantly decreases initially and thereafter increases for both the unitary 
silver nanoparticle $\left(\phi_{1}\right)$ and for hybrid nanoparticle $\left(\phi_{1}+\phi_{2}\right)$ cases. Following the same trend as wall shear stress, a lower volumetric flow rate is computed for hybrid nanofluid compared with silver nanofluid.

Figure (15)-(16) illustrate the distributions in the wall shear stress and volumetric flow rate profiles for different combinations of hybrid nanoparticles. The patterns reveal that on increasing the nanoparticle concentrations, both oscillatory profiles first decrease in magnitude until a particular time, and subsequently increase with time.

Figures (17)-(18) depict the response in wall shear stress and volumetric flow rate for different values of Grashof number and two values of magnetic parameter (Hartmann number). Both quantities are enhanced by increasing $G r$, indicating that stronger thermal buoyancy (free convection effect) accelerates the flow in the arterial domain. However as noted earlier, flow deceleration is induced with stronger magnetic field effect $(M=2$ corresponds to twice the Lorentzian body force relative to viscous hydrodynamic force, compared with $M=1$ ). Flux is therefore depleted in the regime and the blood shears at lower velocity past the arterial boundary. Figure (19)- (20) portrays the effect of variable hybrid nanofluid viscosity on the transient evolution in wall shear stress and flow rate, respectively. Both quantities are increasing functions of nanofluid viscosity and the periodic nature of the pulsatile flow is clearly reproduced in both figures.

Figures (21) - (22) depict the evolution over time in wall shear stress (WSS) and flow rate. It is observed that both these quantities exhibit an inversely proportionality to magnetic field parameter and the oscillatory characteristics of pulsatile flow. Wall shear stress is strongly suppressed with greater Hartmann number $(M)$. The case of non-magnetic i.e. electrically non-conducting blood $(M=0)$. therefore produces the highest velocities. Similarly, volumetric flow rate is also strongly depleted with increasing radial magnetic field, indicating that blood flow is strongly impeded with increment magnetohydrodynamic body force. Peak values of both quantities also arise for early time i.e. $t \sim 1$.

Figures (23)-(24) illustrate the time-varying profiles of wall shear stress and volumetric flow rate respectively for various values of Prandtl number $(P r)$ and Hartmann magnetic parameter $(M)$ also. Both wall shear stress and flow rate are diminished with elevation in Prandtl number and magnetic field parameter. Again, the lowest magnitudes are computed for blood $(\operatorname{Pr}=21)$ compared with water $(\operatorname{Pr}=7)$ as the base fluid. 
Figures (25)-(26) display transient distributions for wall shear stress and volumetric flow rate, with increment in Reynolds number $(R e)$ and magnetic field parameter $(M)$. Strongly periodic profiles are again computed over a wide range of times. Generally, a consistent suppression in wall shear stress is observed with both increasing Reynolds number and higher magnetic field, confirming the results computed earlier for axial velocity. Strong hemodynamic deceleration is therefore induced with greater Lorentzian body force and although inertial effects are greater at higher Reynolds number, the dominant effect is flow retardation and decreased flux.

Figure (27) - (28) shows the influence of slip velocity parameter on wall shear stress and volumetric flow rate profile respectively. Nubar [48] was among the first researchers to identify the presence of slip flow near vessel walls conveying blood, which is a possible cause of the socalled "anomalous" flow behavior of blood near boundaries. Inclusion of hydrodynamic slip is therefore an important feature in more realistic blood flow models. Inspection of Figure (27) reveals that with increasing slip velocity parameter, the wall shear stress decreases significantly, with weak backflow (flow reversal) i.e. negative velocities induced at very low radial coordinate values. In other words, the starting value of wall shear stress profiles is decreased by a fixed value on increasing the magnitude of wall slip. Figure (28) shows that a much more prominent enhancement in volumetric flow rate is generated with increasing wall slip parameter i.e. significant boost in flux is induced with greater wall slip. Flow rate is found to be maximum for the highest value of slip velocity parameter and achieves a minimal magnitude for the classical noslip case $\left(w_{s}=0\right)$ associated with the traditional Navier-Stokes model.

Figure 29 depicts the influence of the $B_{1}$ pressure parameter on pressure gradient. The graph discloses that on increasing the value of $B_{1}$, the peak magnitude computed over the pressure gradient cycle is also shows markedly elevated, and the effect is most pronounced at low and high times.

Figure 30 depicts the effect of $c_{1}$ parameter on pressure gradient profiles. On taking different values of parameter, it can be seen that on increasing the $c_{1}$ value, the length of pressure gradient cycle is reduced, or it can be presented as, for higher $c_{1}$, frequency of cycles is increased. For $c_{1}=$ 0.5 a monotonic decay is computed over time. However, for $c_{1}=1$ the onset of oscillatory behaviour is observed, at larger time values. Fully periodic flow is computed however for $c_{1}=2$, 3, with several cycles observed. 
To analyze the behaviour of blood particles along the axial direction, blood flow patterns are drawn in Figure 31: panels (a) - (i). Panel (a) shows the hemodynamic pattern for the case when nanoparticles are not included with the default values of other parameters. To calculate the impact of variation in nanoparticle concentrations on flow patterns, panels (b) - (c) may be compared with panel (a). Panel (b) displays the pattern for the unitary nanofluid case or for silver nanoparticle inclusion with the other default parameters value. Similarly, Panel (c) presents the changes generated with deployment of hybrid nanoparticles. Inspection of these three figures, clearly demonstrates that hybrid nanoparticles decrease the velocity in the stenotic regime compared with the other cases. For the remaining panels (d)- (i), a comparison may be made with panel (c). Panels (d) and (e) correspond to blood flow patterns for a change in heat generation parameter $(\beta)$ from 0.1 to 0.2 and modification in radial magnetic field from $M=1$ (Lorentzian and viscous forces equal) to $M=0$ (vanishing Lorentz force), respectively. Comparison with panel (c), reveals that blood velocity increases weakly with increasing heat generation parameter whereas it exhibits strong acceleration with absence of magnetic field $(M=0)$ since Lorentzian drag force is eliminated in this scenario. Panels (f) and (g) are plotted respectively for $\mathrm{Gr}=0.5$ and $w_{S}=0.0$. On comparing, these panels it may be deduced that the flow pattern is intensified with greater Gr whereas it is stifled for the zero-slip velocity case i.e. flow acceleration is induced with stronger thermal buoyancy effect and flow deceleration throughout the regime with vanishing wall slip effect i.e. the case of no slip at the vessel walls). Panel (h) and (i) demonstrate that with increasing values of Prandtl number and Reynolds number, i.e. $P r=21$ (corresponds to blood) and $R e=5$, blood flow velocity is depleted i.e. flow retardation is generated in the stenotic regime.

Tables 4 and 5 document the values of wall shear stress and volumetric flow rate with a modification in both nanoparticle concentration and magnetic field parameter. These values are registered at time $t=0.76$ and the other emerging parameters are taken as default values. The results show that on increasing the magnitude of magnetic parameter from $M=0$ to $M=1$, wall shear stress and flow rate both exhibit a descending pattern and similarly on increasing the volume concentration, there is a depletion in both wall shear stress and volumetric flow rate. On comparing the results for silver and hybrid (gold-silver) nanofluid, it can be observed that hybrid nanofluid produces lower wall shear stress and volumetric flows rates at any volume fraction compared with unitary silver nanofluid. 
Figure 32 (a)-(b) displays the variation of axial velocity and temperature profiles for a variety of metallic/metallic oxide nanoparticles suspended within blood. A wider variety of hybrid nanoparticles $\left(\mathrm{Cu}, \mathrm{TiO}_{2}, \mathrm{SiO}_{2}\right)$ are considered. From both graphs, it is very clear that the hybrid combination of ( $\mathrm{Ag}$ and $\mathrm{Au})$ achieves superior results i.e. stronger flow acceleration and greater temperature enhancement compared to other hybrid nanoparticle combinations. The $\mathrm{Cu}, \mathrm{TiO}_{2}$, $\mathrm{SiO}_{2}$ hybrid nanoparticle combinations decrease the velocity significantly (induce flow deceleration) although they do achieve higher temperatures than purely base fluid; however, temperature magnitudes are inferior to the Ag-Au hybrid nanoparticle case.

\section{Conclusions}

Inspired by nano-drug delivery applications in magnetically-assisted pharmacological fluid dynamics, for treatment of arterial diseases, a comprehensive theoretical and computational study is presented for time-dependent hybrid nanoparticle-mediate magneto-hemodynamics and heat transfer (Ag-Au/blood) through an artery with an overlapped stenosis under an external radial magnetic field. Wall slip and heat generation effects are included. Reynolds' exponential model is applied in the formulation to simulate the temperature dependency of blood fluid viscosity. For realistic flow situations, the unsteady component of pulsatile pressure gradient is also included. The principal blood flow characteristics (velocity, temperature, wall shear stress, flow rate) at the stenotic region are evaluated by solving the transformed dimensionless conservation equations under suitable physiological initial and boundary conditions with the FTCS (forward time centered space) finite difference method. Validation of the numerical code is achieved by comparison with earlier non-magnetic, constant viscosity hemodynamic studies. The key findings of the present computations may be summarized as:

- The inclusion of hybrid nanoparticles (Ag-Au/blood) within blood decreases the axial velocity more significantly as compared to unitary nanoparticles (Ag/blood).

- By applying a stronger external magnetic field in the radial direction (i.e. higher Hartmann number) the axial hemodynamic flow is decelerated; however no tangible modification in temperature profile is computed with increasing magnetic field.

- Enhancement in thermal buoyancy effect as simulated via Grashof number $(G r)$ and increasing viscosity parameter $\left(\eta_{0}\right)$ both induce considerable axial flow acceleration in the stenotic regime. 
- Increasing heat generation parameter produces a very weak enhancement in velocity profile whereas it generates a sizeable elevation in temperature magnitudes.

- An increment in slip velocity parameter increases the axial velocity magnitudes strongly but exerts no significant impact on temperature.

- Temperature magnitudes are depressed with rising values of Prandtl number $(\operatorname{Pr})$ i.e. lower thermal conductivity and with increasing Reynolds number $(R e)$ i.e. greater inertial force in the stenotic regime.

- On increasing the value of pressure gradient parameter $\left(B_{1}\right)$, velocity is increased whereas no tangible modification is computed in temperature.

- The wall shear stress and flow rate values have substantially greater magnitudes for hybrid nanofluids compared with unitary nanofluids.

- With time variation, the pressure gradient is found to be lower in coronary (small) arteries compared with large (e.g. femoral) arteries.

- The comparison for distinct hybrid nanofluids indicates that (Ag-Au/-nanoparticle doped blood) produces more beneficial results for nano-drug delivery therapies since it achieves improved hemodynamic flow control and higher temperatures in streaming blood.

The present FTCS code has proved to be a powerful numerical approach for simulating complex nano-pharmacodynamic flows in diseased arteries. In the current simulations, although temperature-dependent viscosity is addressed, however, more complex non-Newtonian characteristics of the blood were not explicitly considered. Hence future studies will consider alternative rheological models for magnetized blood flow e.g. the Sisko model [13], Cross model [41], Carreau model [49]. Moreover, shape factor effects of the nanoparticles have also been ignored in this study. Furthermore, since the present article is restricted to rigid wall arterial vessels, hence deformability of the physiological vessel wall i.e. fluid structure interaction, has not been considered. This may also be addressed with using a variety of approaches e.g. hybrid FSI [50]. Additionally, this study is limited to the case of an overlapping non-tapered artery and may be generalized in the future to examine both converging and diverging arteries. Efforts in all these directions will be communicated imminently. 


\section{Acknowledgements}

The authors are grateful to the Science and Engineering Research Board (SERB), Department of Science and Technology (DST), Government of India for financial support in undertaking the current research work under the research project File Number: ECR/2017/001053 dated $12 / 03 / 2018$. All the authors are grateful to the reviewers for their useful comments which have served to improve the present work.

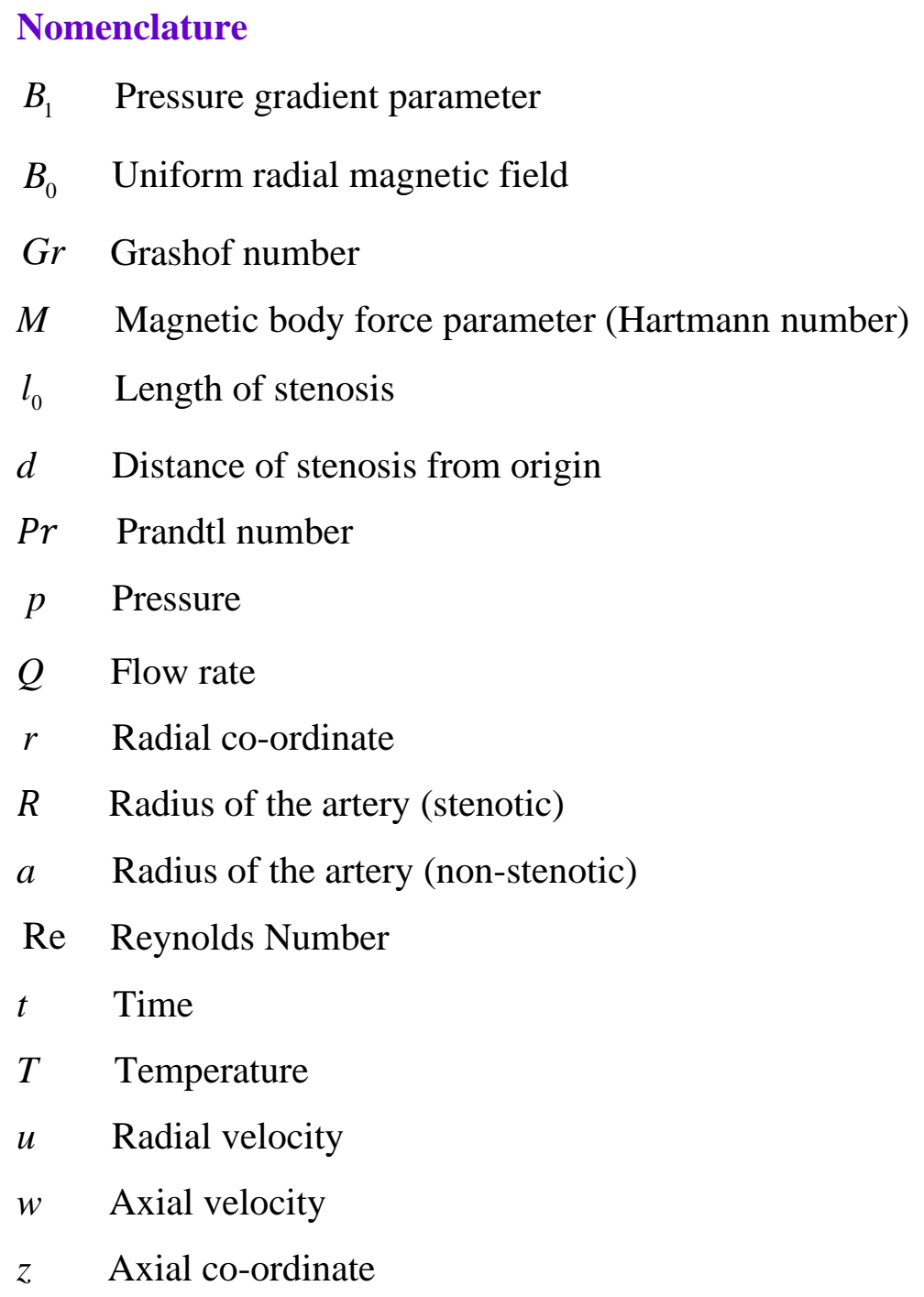

Nomenclature

$B_{1} \quad$ Pressure gradient parameter

$B_{0} \quad$ Uniform radial magnetic field

Gr Grashof number

$M \quad$ Magnetic body force parameter (Hartmann number)

$l_{0} \quad$ Length of stenosis

$d \quad$ Distance of stenosis from origin

$\mathrm{Pr} \quad$ Prandtl number

$p \quad$ Pressure

$Q \quad$ Flow rate

$r \quad$ Radial co-ordinate

$R \quad$ Radius of the artery (stenotic)

a Radius of the artery (non-stenotic)

Re Reynolds Number

$t \quad$ Time

$T \quad$ Temperature

$u \quad$ Radial velocity

$w \quad$ Axial velocity

$z \quad$ Axial co-ordinate

\section{Greek Letters}

$\theta \quad$ Dimensionless temperature

$\beta \quad$ Heat generation parameter 
$\mu_{0} \quad$ Reference viscosity

$\rho \quad$ Density

$\phi \quad$ Nanoparticle concentration

$C_{p} \quad$ Heat capacitance

$k \quad$ Thermal conductivity

$\eta_{0} \quad$ Viscosity constant

$\delta \quad$ Depth of stenosis

$\gamma \quad$ Thermal expansion coefficient

$\tau \quad$ Wall shear stress (WSS)

\section{References:}

[1] World Health Organization (WHO) (2017) Cardiovascular diseases (CVDs). [Online]. http://www.who.int/mediacentre/factsheets/fs317/en/. Accessed 18 Apr 2018

[2] Young, D. F., \& Tsai, F. Y. (1973). Flow characteristics in models of arterial stenoses-I. Steady flow. Journal of Biomechanics, 6(4), 395-410.

[3] Schlichting H. Boundary Layer Theory. McGraw- Hill Book Company, New York. 1968

[4] Biswas D. Blood Flow Models: A Comparative Study. Mittal Publications, New Delhi.2000.

[5] Ling SC, Atabek HB. A nonlinear analysis of pulsatile flow in arteries. J. Fluid Mech.1972; 55(3):493-511.

[6] Chakravarty, S., \& Mandal, P. K. (1994). Mathematical modelling of blood flow through an overlapping arterial stenosis. Mathematical and Computer Modelling, 19(1), 59-70.

[7] Chakravarty, S., Mandal, P. K. (2000). Two-dimensional blood flow through tapered arteries under stenotic conditions. International Journal of Non-Linear Mechanics, 35(5), 779-793.

[8] Mekheimer, K. S., El Kot, M. A. (2012). Mathematical modelling of unsteady flow of a Sisko fluid through an anisotropically tapered elastic arteries with time-variant overlapping stenosis. Applied Mathematical Modelling, 36(11), 5393-5407.

[9] Riahi, D. N., Roy, R., Cavazos, S. (2011). On arterial blood flow in the presence of an overlapping stenosis. Mathematical and Computer Modelling, 54(11-12), 2999-3006

[10] Haghighi, A. R., \& Chalak, S. A. (2017). Mathematical modeling of blood flow through a stenosed artery under body acceleration. Journal of the Brazilian Society of Mechanical Sciences and Engineering, 39(7), 2487-2494.

[11] Srivastav, R. K. (2014). Mathematical model of blood flow through a composite stenosis in catheterized artery with permeable wall. Applications \& Applied Mathematics, 9(1). 
[12] Zaman, A., Ali, N., Bég, O. Anwar. (2016). Unsteady magnetohydrodynamics blood flow in a poroussaturated overlapping stenotic artery—numerical modeling. Journal of Mechanics in Medicine and Biology, 16(04), 1650049.

[13] Ali, N., Zaman, A., Sajid, M. (2014). Unsteady blood flow through a tapered stenotic artery using Sisko model. Computers Fluids, 101, 42-49.

[14] Choi S U S, Eastman J A. Enhancing thermal conductivity of fluids with nanoparticles. ASME Int Mech Eng Congr Exp 1995;66: 99-105

[15] Tripathi, D., Bég, O. A. (2014). A study on peristaltic flow of nanofluids: Application in drug delivery systems. International Journal of Heat and Mass Transfer, 70, 61-70.

[16] Tripathi, J., Vasu, B., Dubey, A., Gorla, R. S. R., Murthy, P. V. S. N., Bég, O. A., \& Saikrishnan, P. (2020). A review on recent advancements in the hemodynamics of nano-drug delivery systems. Nanoscience and Technology: An International Journal, 11(1).

[17] Vasu, B., Dubey, A., Bég, O. A. (2019). Finite element analysis of non-Newtonian magneto-hemodynamic flow conveying nano-particles through a stenosed coronary artery. Heat Transfer-Asian Research.

[18] Ali, N., Zaman, A., Sajid, M., Bég, O. A., Shamshuddin, M. D., \& Kadir, A. (2018). Numerical simulation of time-dependent non-Newtonian nanopharmacodynamic transport phenomena in a tapered overlapping stenosed artery. Nanoscience and Technology: An International Journal, 9(3).

[19] Nadeem, S., \& Ijaz, S. (2015). Theoretical analysis of metallic nanoparticles on blood flow through stenosed artery with permeable walls. Physics Letters A, 379(6), 542-554.

[20] Ahmed, A., \& Nadeem, S. (2016). The study of $(\mathrm{Cu}, \mathrm{TiO} 2, \mathrm{Al2O})$ nanoparticles as antimicrobials of blood flow through diseased arteries. Journal Molecular Liquids, 216, 615-623.

[21] Dubey, A., Vasu, B., Anwar Bég, O., Gorla, R. S., \& Kadir, A. (2020). Computational fluid dynamic simulation of two-fluid non-Newtonian nano-hemodynamics through a diseased artery with a stenosis and aneurysm. Computer Methods in Biomechanics and Biomedical Engineering, 1-27.

[22] Makishima, A. (2004). Possibility of hybrid materials. Ceram. Jap., 39(2), 90-91.

[23] Ijaz, S., \& Nadeem, S. (2017). Biomedical theoretical investigation of blood mediated nanoparticles (AgAl2O3/blood) impact on hemodynamics of overlapped stenotic artery. Journal of Molecular Liquids, 248, 809821.

[24] Ijaz, S., \& Nadeem, S. (2017). A biomedical solicitation examination of nanoparticles as drug agents to minimize the hemodynamics of a stenotic channel. European Physical Journal Plus, 132(11), 448.

[25] Zaman, A., Ali, N., \& Ali, I. (2018). Effects of nanoparticles (Cu (Copper), Silver (Ag)) and slip on unsteady blood flow through a curved stenosed channel with aneurysm. Thermal Science and Engineering Progress, 5, 482-491.

[26] Ahmed, A., Nadeem, S. (2017). Effects of magnetohydrodynamics and hybrid nanoparticles on a micropolar fluid with 6-types of stenosis. Results in Physics, 7, 4130-4139.

[27] Haik, Y., Pai, V., \& Chen, C. J. (2001). Apparent viscosity of human blood in a high static magnetic field. Journal of Magnetism and Magnetic Materials, 225(1-2), 180-186. 
[28] Yadav, R. P., Harminder, S., \& Bhoopal, S. (2008). Experimental studies on blood flow in stenosis arteries in presence of magnetic field. Ultra Sci., 20(3), 499-504.

[29] Ponalagusamy, R., \& Selvi, R. T. (2015). Influence of magnetic field and heat transfer on two-phase fluid model for oscillatory blood flow in an arterial stenosis. Meccanica, 50(4), 927-943.

[30] Nadeem, S., Ijaz, S., \& Adil Sadiq, M. (2014). Inspiration of induced magnetic field on a blood flow of Prandtl nanofluid model with stenosis. Current Nanoscience, 10(5), 753-765.

[31] Zaman, A., Ali, N., \& Khan, A. A. (2020). Computational biomedical simulations of hybrid nanoparticles on unsteady blood hemodynamics in a stenotic artery. Mathematics and Computers in Simulation, 169, 117-132.

[32] Ellahi, R., Raza, M., \& Vafai, K. (2012). Series solutions of non-Newtonian nanofluids with Reynolds' model and Vogel's model by means of the homotopy analysis method. Mathematical and Computer Modelling, 55(78), 1876-1891.

[33] Zaman, A., Khan, A. A., \& Ali, N. (2018). Modelling of unsteady non-Newtonian blood flow through a stenosed artery: with nanoparticles. Journal of the Brazilian Society of Mechanical Sciences and Engineering, 40(6), 307.

[34] Devi, S. A., \& Devi, S. S. U. (2016). Numerical investigation of hydromagnetic hybrid $\mathrm{Cu}-\mathrm{Al} 2 \mathrm{O} 3 /$ water nanofluid flow over a permeable stretching sheet with suction. International Journal of Nonlinear Sciences and Numerical Simulation, 17(5), 249-257.

[35] A.C. Burton, Physiology and Biophysics of the Circulation - Introductory Text, Year Book Medical Publisher, Chicago, 1966.

[36] Hoffman, K. A., Chiang, S. T. (2000). Computational Fluid Dynamics. Engineering Education System, Kansas, 1, 486.

[37] Bég, O. A., Ali, N., Zaman, A., Bég, E. T., \& Sohail, A. (2016). Computational modeling of heat transfer in an annular porous medium solar energy absorber with the P1-radiative differential approximation. Journal of the Taiwan Institute of Chemical Engineers, 66, 258-268.

[38] Bég, O. A., Zaman, A., Ali, N., Gaffar, S. A., \& Bég, E. T. (2019). Numerical computation of nonlinear oscillatory two-immiscible magnetohydrodynamic flow in dual porous media system: FTCS and FEM study. Heat Transfer-Asian Research, 48(4), 1245-1263.

[39] Zaman, A., Ali, N., \& Bég, O. A. (2016c). Numerical study of unsteady blood flow through a vessel using Sisko model. Engineering Science and Technology, an International Journal, 19(1), 538-547.

[40] Ali, N., Javid, K., Sajid, M., \& Anwar Bég, O. (2016a). Numerical simulation of peristaltic flow of a biorheological fluid with shear-dependent viscosity in a curved channel. Computer Methods in Biomechanics and Biomedical Engineering, 19(6), 614-627.

[41]Zaman, A., Ali, N., Bég, O. A., \& Sajid, M. (2016d). Heat and mass transfer to blood flowing through a tapered overlapping stenosed artery. International Journal of Heat and Mass Transfer, 95, 1084-1095.

[42] Ali, N., Javed, M. A., Bég, O. A., \& Hayat, T. (2016b). Mathematical model for isothermal wire-coating from a bath of Giesekus viscoelastic fluid. Chemical Engineering Communications, 203(10), 1336-1348.

[43] Mitvalský, V. Heat transfer in the laminar flow of human blood through tube and annulus. Nature, 206, 307 (1965). 
[44] Diller, K.R., Fundamentals of Bio-Heat Transfer, in Physics of Thermal Therapy: Fundamentals and Clinical Applications. ed. by E.G. Moros, Springer Press, 3-22 (2013).

[45] Hensley, D.W., Mark, A.E., Abella J.R., Netscher, G.M., Wissler, E.H., and Diller, K.R., 50 Years of computer simulation of the human thermoregulatory system, ASME Journal of Biomechanical Engineering. 135, 021005, 1-9 (2013).

[46] Rylander, M.N, Feng, Y., Bass, J. And Diller, K.R., Heat shock protein expression and injury optimization for laser therapy design, Lasers in Surgery and Medicine, 39, 2007, 731-746.

[47]X. Zhang et al., Modelling and simulation on heat transfer in blood vessels subject to a transient laser irradiation, ASME J. Heat Transfer. 142(3): 031201 (9 pages) (2020).

[48] Y. Nubar, Blood flow, slip, and viscometry, Biophys J. 11(3): 252-264 (1971).

[49]Z. Asghar, N. Ali, M. Sajid and O. Anwar Bég, Micro-organism swimming propulsion through a shear ratedependent biorheological fluid in an active channel assisted by a magnetic field, J. Magnetism and Magnetic Materials (2019). doi.org/10.1016/j.jmmm.2019.165283 (13 pages)

[50] Tezduyar TE, Sathe S, Schwaab M, Conklin BS, Arterial fluid mechanics modeling with the stabilized spacetime fluid-structure interaction technique. Int. J. Numer. Methods Fluids 57:601-629 (2008).

[51] O. Anwar Bég, B. Islam, MD. Shamshuddin and T. A. Bég, Computational fluid dynamics analysis of moisture ingress in aircraft structural composite materials, Arabian J. Science Engineering (2019). doi.org/10.1007/s13369-019-03917-4 (23 pages)

[52] O. Anwar Bég, Tasveer A. Bég, J. Patterson, B. Vasu, S. Kuharat, Ali Kadir and W.S. Jouri, Two-way fluidstructure interaction hydroelastic simulation of vibrating membranes with applications in marine renewable energy, ICMEAMT 2020: $14^{\text {th }}$ International Conference on Mechanical Engineering, Applied Mechanics and Technology, Cairo, Egypt, December 14-15 (2020). 